Aus der Abteilung Pädiatrie III mit dem Schwerpunkt Pädiatrische Kardiologie und Intensivmedizin

(Prof. Dr. med. T. Paul)

im Zentrum Kinderheilkunde und Jugendmedizin der Medizinischen Fakultät der Universität Göttingen

\title{
Postoperatives Monitoring der regionalen Lungenventilation durch die Elektrische Impedanztomographie bei Kindern und Jugendlichen mit einem angeborenen Herzfehler
}

\author{
INAUGURAL-DISSERTATION \\ zur Erlangung des Doktorgrades \\ der Medizinischen Fakultät \\ der Georg-August-Universität zu Göttingen \\ vorgelegt von \\ Kristin Eva Becker \\ aus \\ Wilhelmshaven
}

Göttingen 2012 
Dekan: Prof. Dr. med. C. Frömmel

I. Berichterstatter: Prof. Dr. med. Paul (Betreuer)

II. Berichterstatter: Priv.-Doz. Dr. med. Hinz (Korreferent)

III. Berichterstatter: Prof. Dr. med. Andreas (Drittreferent)

IV. Berichterstatter: Prof. Dr. med., Dr. rer. nat. Crozier (Promotor-Vertretung)

Tag der mündlichen Prüfung: 13.06.2012 


\section{Inhaltsverzeichnis}

$1 \quad$ Einleitung 1

1.1 Etablierte Methoden zur Überwachung der Beatmung 2

1.2 Bedarf einer neuen Methode zur Überwachung der regionalen Lungenventilation 3

1.3 Funktionsprinzipien der EIT $\quad 4$

1.4 Historische Entwicklung der EIT und Stand der Forschung 4

1.5 Fragestellung $\quad 7$

2 Patienten und Methoden 10

$\begin{array}{ll}2.1 \text { Zeitraum und Ort der Studie } & 10\end{array}$

2.2 Probandenrekrutierung 10

2.2.1 Anzahl und Charakterisierung der Probanden 10

2.2.2 Einschlusskriterien 11

2.2.3 Ausschlusskriterien 11

2.2.4 Behandlung der Probanden 11

2.3 EIT und verwendetes EIT-System 12

$\begin{array}{ll}2.4 \text { Versuchsdurchführung } & 17\end{array}$

2.5 Versuchsdokumentation $\quad 20$

2.6 Auswertung der Daten $\quad 21$

2.7 Statistische Analyse $\quad 26$

3 Ergebnisse $\quad 27$

3.1 Verteilung der relativen Impedanzänderung während maschineller Beatmung und nach Extubation $\quad 27$

3.2 Darstellung des rechten und linken Lungenflügels 30

3.3 Darstellung der ventralen und dorsalen Lungenbereiche 32

3.4 Messungen über mehrere Tage 32

3.5 Visualisierung von Beatmungsmanövern und therapeutischen Eingriffen 33

3.6 Darstellung der Ergebnisse für das Patientenkollektiv 37

3.6.1 Bedeutung von maschineller Beatmung, Spontanatmung und individuellen Patientencharakteristika für die relative Impedanz$\begin{array}{ll}\text { änderung } & 38\end{array}$

3.6.2 Relative Impedanzänderung während Beatmung und Spontanatmung 39 
3.6.2.1 Mittelwerte und prozentuale Änderung

3.6.2.2 Variation der Werte der relativen Impedanzänderung der Patienten unter Beatmung und unter Spontanatmung

3.6.2.3 Unterschiede der relativen Impedanzänderung unter Beatmung und Spontanatmung

3.6.3 Geschlechtsunterschiede

3.6.4 Seitenvergleich rechter Lungenflügel - linker Lungenflügel

3.6.5 Unterschiede zwischen ventralen und dorsalen Lungenbereichen

3.6.6 Abhängigkeit der relativen Impedanzänderung vom Alter

3.6.7 Korrelation des $\mathrm{CO}_{2}$-Partialdruckes mit der relativen Impedanzänderung

3.6.8 Zusammenhänge zwischen der relativen Impedanzänderung und den Beatmungsparametern

\section{Diskussion}

4.1 Bewertung und Auswertung der Daten dieser Arbeit

4.2 Erörterung der Ergebnisse dieser Arbeit

4.3 Anwendung der EIT bei herzoperierten Kindern

4.4 Schlussfolgerungen und Ausblick

5 Zusammenfassung

$6 \quad$ Anhang

$7 \quad$ Literaturverzeichnis 


\section{Einleitung}

Im klinischen Altag ist das Wissen über regionale Ventilationsverhältnisse vor allem bei Patienten mit Pathologien des respiratorischen Systems und bei maschinell beatmeten Patienten zur Überwachung und Optimierung der Therapie von großer Bedeutung, da pathologische Prozesse, wie z.B. Atelektasen und Emphyseme, die Lunge zumeist nicht als Ganzes betreffen.

Im Gegensatz zur Spontanatmung, bei der ein Unterdruck durch die Expansion von Thorax und Lunge für den Luftstrom in die Lunge verantwortlich ist, ist die maschinelle Beatmung in der klinischen Praxis eine Überdruckbeatmung. Diese unphysiologischen Druckverhältnisse unter maschineller Beatmung führen zu einer Beeinträchtigung der Lungenfunktion und zu inhomogenen Belüftungsverhältnissen vor allem in den abhängigen, unten liegenden Lungenarealen: Das Zwerchfell wird durch den Beatmungsdruck passiv nach kaudal bewegt; aufgrund des geringeren Widerstandes geschieht dies beim auf dem Rücken liegenden Patienten stärker in den ventralen als in den dorsalen Arealen. Daraus resultiert eine schlechtere Belüftung der dorsalen Lungenareale [Oczenski 2008]. Unter einer maschinellen Beatmung kann neben dieser ohnehin schon inhomogenen Belüftung mit Neigung zur Bildung von Mikroatelektasen eine Vielzahl von weiteren Problemen auftreten, so dass die Lunge unter einer inadäquaten Beatmungstherapie erheblichen Schaden nehmen kann [Amato et al. 1998, Oczenski 2008]: Zu hohe Beatmungsdrücke schädigen die Lungenstruktur und führen zum so genannten Barotrauma der Lunge, zu hohe Volumina führen zum so genannten Volutrauma. Ein zu hoch eingestellter PEEP (Positive End-Expiratory Pressure) resultiert in einer Überblähung der Alveolen. Regionaler Kollaps und Überblähung können innerhalb von Sekunden auftreten [Neumann et al. 1998]. Ziel einer optimalen Beatmungstherapie ist die lungenprotektive Beatmung, d.h. atelektatische Regionen zu öffnen und primär nicht atelektatische Areale durch die künstliche Beatmung so wenig wie möglich zu traumatisieren. Um diesem Ziel gerecht werden zu können, ist es vorteilhaft, kontinuierlich Informationen über die aktuellen regionalen Ventilationsverhältnisse in der Lunge zu erhalten. So können Inhomogenitäten der Ventilation erkannt werden und die Beatmung an die jeweiligen Bedürfnisse des Patienten individuell angepasst werden. 
Leider gibt es bisher kein in der pädiatrischen Intensivmedizin klinisch etabliertes Verfahren, welches die regionalen Belüftungsverhältnisse in der Lunge kontinuierlich erfasst und somit ein frühzeitiges Erkennen von inhomogener Belüftung, inkompletter Lungenentfaltung, intrapulmonalen Flüssigkeitsansammlungen sowie von Überblähungen oder Kollaps einzelner Lungenareale ermöglicht und zur direkten Beurteilung der Beatmungstherapie herangezogen werden kann.

\subsection{Etablierte Methoden zur Überwachung der Beatmung}

Die derzeit etablierten Routine-Methoden zur Überwachung einer Beatmungstherapie und deren Erfolg sind zum größten Teil nicht geeignet, regionale Ventilationsstörungen frühzeitig zu erkennen, d.h. sie geben nur Auskunft über die Funktion und die Ventilationssituation der gesamten Lunge, ohne Informationen über mögliche regionale Inhomogenitäten widerzuspiegeln. Dazu gehören die intermittierend durchgeführten arteriellen und venösen Blutgasanalysen sowie die kontinuierliche transkutane Pulsoxymetrie [Caples und Hubmayr 2003], die zur Überwachung des effektiven Gasaustausches dienen. Ebenso sind die Ergebnisse der Messung der Ventilationsparameter wie der Beatmungsdrücke, Gasflüsse, Atemzug - und Atemminutenvolumina sowie die Messung einfacher spirometrischer Parameter nur von globalem Charakter.

Die regionale Lungenventilation kann nur mit einer geringen Anzahl medizinischer Untersuchungsmethoden erfasst werden, die aber in ihrer Aussagekraft und regelmäßigen Durchführung insgesamt begrenzt sind. Die einfachsten Methoden sind Inspektion und Auskultation, die zur schnellen Erkennung von Beatmungsproblemen sehr wertvoll sind. Jedoch hängen beide vom Untersucher und seiner Erfahrung ab und geben zudem nur einen groben, nicht objektivierbaren Hinweis auf regionale Ventilationsunterschiede.

An bildgebenden Verfahren kommen vor allem konventionelle Röntgenuntersuchungen sowie als Schnittbildgebung Computertomographie (CT) und Magnetresonanztomographie (MRT) zum Einsatz, die jedoch nur Momentaufnahmen der aktuellen Situation darstellen und/oder aufgrund der Strahlenbelastung und des zum Teil erheblichen Untersuchungsaufwandes nicht beliebig wiederholbar sind. Zur Durchführung einer CT- oder MRT-Untersuchung ist der Transport des Patienten notwendig, wodurch der kritisch kranke Patient unter 
Umständen gefährdet wird [Smith et al. 1990, Warren et al. 2004]. Zu einer kontinuierlichen, bettseitigen Überwachung der regionalen Lungenventilation können diese Methoden aus den genannten Gründen somit nicht dienen.

Des Weiteren existieren noch spezielle Verfahren zur Darstellung der regionalen Ventilation, die jedoch aufgrund von erheblicher Strahlenbelastung und großem apparativen Untersuchungsaufwand ebenfalls nicht routinemäßig und teilweise nur unter experimentellen Bedingungen eingesetzt werden. Hier sind das Ein- und Auswaschverfahren unter Verwendung von inerten Gasen [Hinz et al. 2003a, van Genderingen et al. 2004], die Ventilationsszintigraphie [Hinz et al. 2003b] sowie die Single-Photon-Emissions-Computertomographie (SPECT) [Hinz et al. 2003b] und die Positronen-Emissions-Tomographie (PET) [Richard et al. 2009] zu nennen.

\subsection{Bedarf einer neuen Methode zur Überwachung der regionalen Lungenventilation}

Aus den oben genannten Gründen besteht der Bedarf an einer nicht-invasiven Methode, die einfach zu bedienen und ohne Verwendung ionisierender Strahlung sowie ohne erheblichen apparativen Aufwand direkt am Krankenbett anwendbar ist, um eine kontinuierliche Messung der regionalen Ventilationsverhältnisse zu ermöglichen. Sie sollte die zeitliche und räumliche Verteilung der Luft in der Lunge mit hoher lokaler Auflösung über lange Zeiträume hinweg erfassen und zur Darstellung bringen. Die Interpretation der gewonnenen Daten sollte idealerweise bettseitig durch den behandeInden Arzt erfolgen können. Dadurch könnten die bisher etablierten Verfahren sinnvoll ergänzt werden und bei maschinell beatmeten Patienten zusätzliche Informationen über den aktuellen Beatmungszustand sowie die effektive Belüftungssituation der Lunge gewonnen werden.

Die elektrische Impedanztomographie (EIT) ist ein bildgebendes Verfahren, das nicht-invasiv Querschnitte von Körpern aufgrund unterschiedlicher elektrischer Eigenschaften der einzelnen Gewebe in verschiedenen Ebenen darstellen kann. Vorausgegangene tierexperimentelle Studien sowie Studien an gesunden Probanden, erwachsenen Intensivpatienten sowie Früh- und Neugeborenen zeigten, dass mittels EIT sowohl physiologische als auch pathologische Veränderungen der regionalen Lungenventilation erfasst werden können (siehe 1.4). 


\subsection{Funktionsprinzipien der EIT}

Das Grundprinzip der EIT beruht darauf, dass jeder Gewebetyp spezifische elektrische Eigenschaften besitzt [Geddes und Baker 1967], die zu einer inhomogenen Verteilung der elektrischen Leitfähigkeit der Organstrukturen in einer jeweils untersuchten Körperebene führen. Durch die wiederholte Applikation sehr kleiner Wechselströme und die Messung der resultierenden Potentialdifferenzen auf der Körperoberfläche wird die regionale Verteilung der elektrischen Impedanz in der betrachteten Körperebene erfasst und rekonstruiert als Tomogramm dargestellt [Barber und Brown 1984].

Aufgrund ihres Funktionsprinzips ist die EIT sehr gut geeignet, die regionale Ventilation der Lunge zu visualisieren, so dass die Beurteilung der regionalen Lungenfunktion bisher zu den bedeutendsten Einsatzgebieten der EIT zählt. Die elektrischen Eigenschaften des Lungengewebes werden wesentlich vom Luftgehalt der Lunge beeinflusst: Aufgrund des periodisch wechselnden Luftgehaltes durch die In- und Exspiration weist die Lunge sehr große Variationen der elektrischen Impedanz auf. Während Luft ein schlechter elektrischer Leiter ist, sind Wasser, Blut und Gewebe gute Leiter für elektrischen Strom. Die vermehrte Dehnung des Lungengewebes durch die Luftfüllung bei Inspiration führt zu kleineren Querschnitten des zwischen den Alveolen liegenden elektrisch leitenden Gewebes und damit zu höheren elektrischen Widerständen. Dieser Mechanismus ermöglicht es, mittels EIT Änderungen im pulmonalen Luftgehalt und in der pulmonalen Luftverteilung $\mathrm{zu}$ ermitteln [Hahn et al. 1995]. Zwischen dem Atemzugvolumen und der relativen Impedanzänderung besteht ein enger Zusammenhang: Die Variation der lokalen Impedanzänderung korreliert positiv mit der regionalen Ventilation und dem regionalen Lungenvolumen und gilt als Maß für das Atemzugvolumen [Erlandsson et al. 2006, Frerichs et al. 2002, Hahn et al. 1995, Harris et al. 1987, 1988, Hinz et al. 2003a, 2003b, McArdle et al. 1988, Meier et al. 2006].

\subsection{Historische Entwicklung der EIT und Stand der Forschung}

Henderson und Webster haben bereits 1978 erstmals elektrische Bioimpedanz bildlich dargestellt [Henderson und Webster 1978]. Ziel war es, ein 
zweidimensionales Bild der transthorakalen Impedanz zu erzeugen. Dies erfolgte durch die Einspeisung eines Wechselstromes mittels einer großflächigen Elektrode und der Messung der Potentiale mittels eines Arrays vieler kleiner Elektroden auf der gegenüberliegenden Seite. Da die Qualität dieser dorsoventralen Bilder sehr schlecht war und deren Interpretation schwierig, setzte sich diese Art der bildlichen Darstellung der tho rakalen elektrischen Bioimpedanz nicht durch.

Die eigentliche EIT wurde von Barber und Brown in den frühen 1980er Jahren an der Universität in Sheffield (Department of Medical Physics and Clinical Engineering) in Großbritannien entwickelt [Barber und Brown 1984]. Da von diesen Wissenschaftlern die ersten tomographischen Bilder der Verteilung der elektrischen Impedanz im Körper stammen, gelten sie als die "Väter der EIT“. Das erste kommerziell erhältliche EIT-Gerät wurde von ihnen konstruiert, das Sheffield APT-System Mark 1 [Brown und Seagar 1987]. Das erste öffentlich gezeigte EIT-Bild war ein Querschnittsbild des menschlichen Unterarmes [Barber und Brown 1984]. Ursprünglich wurde das Verfahren als "Applied Potential Tomography" (APT) bezeichnet, die Bezeichnung EIT setzte sich erst in den neunziger Jahren des 20. Jahrhunderts durch.

Bereits kurz nach der Entwicklung der EIT gab es 1985 verschiedene Vorschläge für deren Anwendung in klinischen Bereichen, wie z.B. die Nutzung für die Messung des Herzzeitvolumens, des Blutflusses durch die peripheren Gefäße sowie auch für die Beurteilung von Atmung und Beatmung [Brown et al. 1985].

In den folgenden 10 Jahren wurden jedoch zunächst kaum relevante experimentelle Validierungsstudien bzw. klinische Untersuchungen mit der EIT durchgeführt. Die Methode kam weitestgehend nur bei gesunden Probanden in unterschiedlichen Anwendungsbereichen zum Einsatz [Brown und Barber 1987, Griffiths et al. 1992, Harris et al. 1987, 1988, Holder 1989, Mc Ardle et al. 1988, Morice et al. 1993, Murphy et al. 1987, Smallwood et al. 1993].

Seit Mitte der neunziger Jahre wurden die Geräte und Geräteeigenschaften sowohl durch tierexperimentelle als auch klinische Studien für einen künftigen Klinikeinsatz immer weiter optimiert. Dies geschah vor allem auf dem Gebiet des Herz-KreislaufSystems [Vonk Noordegraaf et al. 1996], der Lungenventilation [Adler et al. 1997, 1998, Campbell et al. 1994, Eyübog lu et al. 1995, Frerichs et al. 1996, 1998a, 1998b, 1999a, 1999b, Hahn et al. 1995, 1996, Kunst et al. 1998a, 1998b, 1999a, 1999b], der Magenmotilität [Vaisman et al. 1999] und der Hirnaktivität [Holder et al. 1996, 1999]. 
Aufgrund der gut zu registrierenden elektrischen Eigenschaften des Lungengewebes durch Änderungen im regionalen Luftgehalt wurde die Nutzung der EIT zur regionalen Lungenfunktionsbeurteilung als besonders interessant und aussichts reich angesehen und gewann stetig an Bedeutung. Die ET wurde seitdem zunehmend in tierexperimentellen Studien [Adler et al. 1997, 1998, Frerichs et al. 1998b, 1999a, 2002, 2003b, 2007, Hahn et al. 1995, 2006, Hinz et al. 2003b, Kunst el al. 2000a, 2000b, Meier et al. 2006, 2008, Rooney 2009, van Genderingen et al. 2003, 2004, Wrigge et al. 2008], in Studien an gesunden Probanden [Frerichs et al. 1996, 2003a, 2004, Hahn et al. 1995, 1996, Marquis et al. 2006, Reifferscheid et al. 2011, Schibler et al. 2009], erwachsenen Intensivpatienten [Bikker et al. 2009, 2011, Costa et al. 2009, Frerichs et al. 1998a, Hinz et al. 2003a, 2005, Kunst et al. 1999a, 1999b, Lowhagen et al. 2011, Victorino et al. 2004] sowie Früh- und Neugeborenen [Brown et al. 2002, Frerichs et al. 2001, 2003a, Hampshire et al. 1995, Heinrich et al. 2006, Marven et al. 1996, Pham et al. 2011, Riedel et al. 2009, Schibler et al. 2009, Smallwood et al. 1999] als experimentelle, nicht-invasive Methode genutzt, um die Methode zur Überwachung und Beurteilung physiologischer und pathologischer Veränderungen der regionalen Lungenventilation zu validieren.

Tierexperimentell wurden vor allem die Fähigkeit der Erfassung sowie der Vergleichbarkeit der regionalen Lungenventilation mit Computertomographie (CT) [Frerichs et al. 2002, Hahn et al. 2006, Meier et al. 2006, 2008, Wrigge et al. 2008], Single-Photon-Emissions-Computertomographie (SPECT) [Hinz et al. 2003b], Ventilationsszintigraphie [Hinz et al. 2003b] und Positronen-Emissions-Tomographie (PET) [Richard et al. 2009] validiert. Es konnte gezeigt werden, dass die EIT sowohl globale als auch regionale Ventilation verglichen mit den Verfahren CT, SPECT, Ventilationsszintigraphie und PET mit ausreichender Genauigkeit darstellt, um klinische Fragestellungen beantworten zu können. Victorino et al. (2004) und Costa et al. (2009) bestätigten die gute Vergleichbarkeit der EIT mit CT an erwachsenen, maschinell beatmeten Intensivpatienten. Dass pathologische Zustände der Lunge mit der EIT erfasst werden können, wurde in tierexperimentellen Studien durch das Induzieren chemischer Lungenschäden und der impedanztomographischen Darstellung deren Auswirkungen gezeigt [Adler et al. 1997, Frerichs et al. 1998b, Hinz et al. 2003b, Kunst et al. 2000a, 2000b, Lindgren et al. 2007, Meier et al. 2008, Richard et al. 2009, van Genderingen et al. 2003, 2004]. Die Effekte verschiedener Beatmungseinstellungen auf die regionale Lungenventilation konnten mit der EIT in 
einigen Arbeiten an Tieren [Adler et al. 1998, Frerichs et al. 1999a, 2002, 2003b, 2007, Lindgren et al. 2007, Meier et al. 2008, Richard et al. 2009], erwachsenen Intensivpatienten [Bikker et al. 2009, 2011, Costa et al. 2009, Frerichs et al. 1998a, 2003a, 2005, Kunst et al. 1999a, Lowhagen et al. 2011] und Neugeborenen [Frerichs et al. 2001] bewertet werden. Der Gewinn von Erkenntnissen über die Ventilationsverteilung bei spontan atmenden Neugeborenen [Hampshire et al. 1995, Pham et al. 2011, Riedel et al. 2009] und in verschiedenen Körperpositionen [Frerichs et al. 2003a, Heinrich et al. 2006, Schibler et al. 2009], bei spontan atmenden Erwachsenen in verschiedenen Körperpositionen [Frerichs et al. 1996, Reifferscheid et al. 2011] und im Vergleich zwischen älteren und jüngeren spontan atmenden Menschen [Frerichs et al. 2004] wurde mit der EIT ermöglicht. Frerichs et al. (1998a) und Karsten et al. (2011) stellten bei erwachsenen Patienten peri- und intraoperative Unterschiede in der Ventilationsverteilung durch EIT-Untersuchungen dar. Humphreys et al. (2011) untersuchten mittels EIT die Ventilationsverteilung bei Kindern mit angeborenem Herzfehler unmittelbar präoperativ während der Narkoseeinleitung, der Intubation und dem Beginn der maschinellen Beatmung.

Die technische Entwicklung der EIT ist seit den neunziger Jahren des 20. Jahrhunderts sehr schnell vorangeschritten [Hahn et al. 2001], Hard- und Software wurden kontinuierlich verbessert und optimiert. Aufgrund dessen wurden zunehmend stabile Messungen in einer Umgebung möglich, in der viele elektrische Geräte parallel betrieben werden, wie dies z.B. auf Intensivstationen der Fall ist. Hierdurch konnte der klinische Einsatz der EIT einen großen Schritt vorangebracht werden und gewann in den letzten 10 Jahren zunehmend an Bedeutung und Interesse. Diese Entwicklung resultierte kürzlich in der kommerziellen Verfügbarkeit eines EITSystems für erwachsene Patienten (Pulmovista 500, Drägerwerk AG, Lübeck, Deutschland).

\section{$1.5 \quad$ Fragestellung}

Im vorherigen Abschnitt wurde dargestellt, dass sich die ET seit ihrer Einführung im Jahre 1980 durch eine Vielzahl von experimentellen Studien deutlich weiterentwickelt hat und nachgewiesen werden konnte, dass sowohl physiologische als auch pathologische Veränderungen der regionalen Lungenventilation erfasst werden. Um die Einsetzbarkeit der EIT bei herzoperierten Kindern zu überprüfen und um die 
Methode entsprechend den Resultaten weiter zu optimieren und zunehmend in den klinischen Alltag auf einer pädiatrischen Intensivstation zu integrieren, sollte in dieser Arbeit die Lungenventilation unter normalen klinischen Bedingungen auf einer pädiatrischen Intensivstation an einem Kollektiv von herzoperierten Kindern untersucht werden. Eine solche Studie bei Kindern unmittelbar nach chirurgischem Thoraxeingriff wurde bisher nicht durchgeführt, zudem ist aus Mangel an geeigneten Untersuchungsmethoden noch wenig über die regionale Lungenventilation bei maschinell beatmeten Kindern bekannt. Bei diesem Kollektiv ist die Tatsache von Bedeutung, dass die Lungenfunktion sowie die Atemmechanik nach medianer Sternotomie und extrakorporaler Zirkulation ohnehin schon eingeschränkt sind [Dalton und Connally, 1993, Johnson et al. 1996]. Die nicht-invasive, strahlenfreie, funktionelle Bildgebung der Lunge mittels EIT bietet hierbei neue Möglichkeiten der Charakterisierung der regionalen Lungenfunktion bei einem Kollektiv an herzoperierten Kindern sowohl unter maschineller Beatmung als auch nach Extubation unter anschließender Spontanatmung.

Die Fragestellungen dieser Arbeit werden wie folgt zusammengefasst:

1. Bestimmung der regionalen Lungenventilation bei Kindern mit angeborenem Herzfehler nach kardiochirurgischem Eingriff unter maschineller Beatmung und unter Spontanatmung;

2. Vergleich der regionalen Lungenventilation unter maschineller Beatmung und unter Spontanatmung;

3. Überprüfung eventuell vorhandener Altersunterschiede in der globalen Lungenventilation unter maschineller Beatmung und unter Spontanatmung;

4. Vergleich der regionalen Lungenventilation in ventralen und dorsalen Lungenbereichen;

5. Untersuchung der regionalen Lungenventilation bei verschiedenen Formen der maschinellen Beatmung bzw. der maschinellen Atemunterstützung sowie in unterschiedlichen klinischen Situationen;

6. Beurteilung der Anwendbarkeit der EIT im klinischen Alltag auf einer pädiatrischen Intensivstation im Routinestationsablauf;

7. Prüfung der Relevanz der EIT-Befunde für klinische Entscheidungen und Therapie. 
Da diese Studie auch der weiteren Validierung der EIT als Verfahren zur kontinuierlichen, nicht-invasiven Überwachung der regionalen Lungenventilation in der klinischen Routine auf einer pädiatrisch-kardiologischen Intensivstation dienen soll, kann sie zur Etablierung der Methode in dieser speziellen klinischen Anwendung beitragen. 


\section{Patienten und Methoden}

\subsection{Zeitraum und Ort der Studie}

Bei der vorliegenden Arbeit handelt es sich um eine prospektive klinische Studie, die über den Zeitraum von 2 Jahren auf der pädiatrischen Intensivstation der Abteilung für Pädiatrische Kardiologie und Intensivmedizin im Zentrum Kinderheilkunde der Universitätsmedizin der Georg-August-Universität Göttingen durchgeführt wurde.

\subsection{Probandenrekrutierung}

\subsubsection{Anzahl und Charakterisierung der Probanden}

Die Studie wurde an 30 Patienten mit einem angeborenem Herzfehler im Alter von 0 bis 18 Jahren, die sich zur primären Korrekturoperation oder zu einer Re-Operation in der Klinik befanden, durchgeführt. Davon waren 10 Patienten weiblichen und 20 männlichen Geschlechts; das Alter lag im Mittel bei 5 Jahren. Das Patientenkollektiv wurde in folgende drei Altersgruppen eingeteilt: Säuglinge (0-1 Jahr, $n=7)$, Kleinkinder (1-3 Jahre, $\mathrm{n}=6$ ) und Kindergarten- und Schulkinder/ Jugendliche (4-18 Jahre, $n=17)$. Auf eine zusätzliche Altersgruppe jenseits des 13./14. Lebensjahres wurde verzichtet, da in dieser nur ein Patient vertreten gewesen wäre.

Tabelle 1: Präoperative Diagnosen $(n=30)$.

\begin{tabular}{|l|c|c|}
\hline Diagnosen & Primäre OP & Re-OP \\
\hline Ventrikelseptumdefekt (VSD) & $\mathrm{n}=5$ & - \\
\hline Vorhofseptumdefekt (ASD) & $\mathrm{n}=4$ & - \\
\hline Atrio-ventrikulärer Septumdefekt (AVSD) & $\mathrm{n}=5$ & - \\
\hline Fallot'sche-Tetralogie & $\mathrm{n}=1$ & $\mathrm{n}=4$ \\
\hline DORV (Double Outlet Right Ventricle) & - & $\mathrm{n}=2$ \\
\hline Aortenklappenstenose /-insuffizienz & $\mathrm{n}=4$ & $\mathrm{n}=1$ \\
\hline Truncus arteriosus communis & - & $\mathrm{n}=1$ \\
\hline d-Transposition der großen Arterien (d-TGA) & - & $\mathrm{n}=1$ \\
\hline Pulmonalarterienstenose & - & $\mathrm{n}=1$ \\
\hline Mitralinsuffizienz & $\mathrm{n}=1$ & - \\
\hline
\end{tabular}




\subsubsection{Einschlusskriterien}

In die Studie aufgenommen wurden postoperativ beatmete Kinder mit angeborenem Herzfehler beiderlei Geschlechts vom Neugeborenenalter bis zur Vollendung des 18. Lebensjahres. Voraussetzung waren die endotracheale Intubation (entweder nasooder orotracheal) und maschinelle Beatmung der Patienten bei Aufnahme auf die pädiatrische Intensivstation. Vor Aufnahme in die Studie erfolgte die mündliche und schriftliche Einwilligung der Eltern nach entsprechender Aufklärung durch einen der behandelnden Ärzte. Für die Durchführung der Studie lag ein positives Votum der Ethik-Kommission der Universitätsmedizin Göttingen vor.

\subsubsection{Ausschlusskriterien}

Nicht in die Studie aufgenommen wurden Kinder, deren Thorax postoperativ nur provisorisch mit einem Goretex-Flicken verschlossen war, Kinder mit schweren kongenitalen Malformationen und Kinder, deren Eltern die Aufnahme in die Studie verweigerten.

\subsubsection{Behandlung der Probanden}

Aufgrund der geplanten operativen Behandlung ihres angeborenen Herzfehlers waren die Patienten bereits hospitalisiert. Die Intubation der Patienten erfolgte präoperativ im Rahmen der Narkoseeinleitung durch die Anästhesie. Postoperativ wurden die Patienten intubiert und maschinell beatmet auf die pädiatrische Intensivstation verlegt und dort unmittelbar nach Übernahme in die Studie eingeschlossen. Die Beatmung erfolgte je nach Körpergewicht mit den Beatmungsgeräten Babylog 8000 (Körpergewicht < $10 \mathrm{~kg}$ ) oder Evita 4 Neoflow (Körpergewicht $>10 \mathrm{~kg}$, beide Beatmungsgeräte von der Drägerwerk AG, Lübeck, Deutschland). Die verwendeten Beatmungsmodi waren SIMV (Synchronized Intermittent Mandatory Ventilation), BIPAP (Biphasic Positive Airway Pressure) sowie CPAP (Continuous Positive Airway Pressure).

Die Patienten wurden durch eine mediane Sternotomie operiert und waren mit mindestens zwei thorakalen Wunddrainagen (Pleura- und/ oder Substernal- und 
Perikarddrainage) versorgt. Außerdem waren intraoperativ bei allen Patienten routinemäßig temporäre epimyokardiale Schrittmacherelektroden (entweder Vorhof und Ventrikel oder nur Ventrikel) zur Behandlung postoperativer bradykarder Herzrhythmusstörungen ge legt worden.

Nach Aufnahme in die Studie erfolgten alle therapeutischen Maßnahmen wie üblich im Rahmen der klinischen Routineversorgung; zusätzlich wurden nicht-invasiv am Krankenbett EIT-Messungen (siehe 2.4) durchgeführt, bis die Patienten extubiert werden konnten und sich danach stabile respiratorische Verhältnisse (Eupnoe, ausgeglichene Blutgasanalyse) eingestellt hatten.

\subsection{EIT und verwendetes EIT-System}

Prinzip der EIT ist es, durch die Messung von Spannungsdifferenzen an der Oberfläche elektrisch leitfähiger Körper (z.B. des menschlichen Körpers) die Impedanzverteilung im Körperinneren darzustellen und hieraus ein zweidimensionales Schnittbild zu berechnen. Hierzu wird zirkulär an verschiedenen Stellen der Schnittebene mit Hilfe von Elektroden ein Wechselstrom eingespeist sowie die resultierenden Potentialdifferenzen zwischen zwei Elektrodenpaaren gemessen [Barber und Brown 1984]. Zur Beurteilung der Impedanzverteilung im Thorax und damit der Ventilationsverhältnisse in der Lunge der Probanden lag die Schnittebene in der vorliegenden Arbeit etwa 1-2 cm unterhalb der Mamillarebene (siehe Abb. 1a und 1b). 

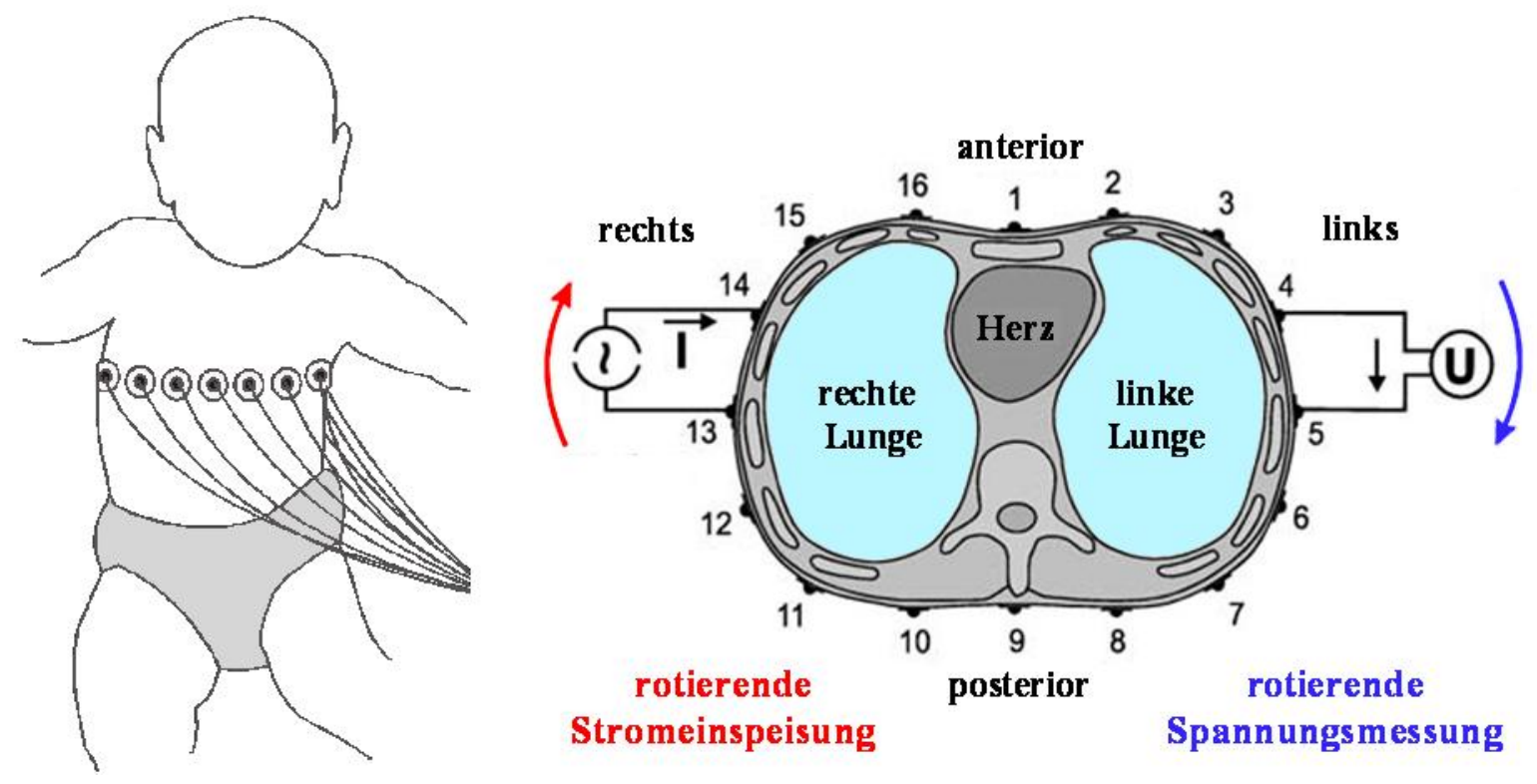

Abbildung 1a: Schematische Darstellung der Anbringung der EKG-Elektroden am Thorax eines Kindes (links) und der rotierenden, zyklischen Stromeinspeisung (I) und Spannungsmessung (U) (rechts) (modifiziert nach Frerichs et al. (2005, S. 68)).

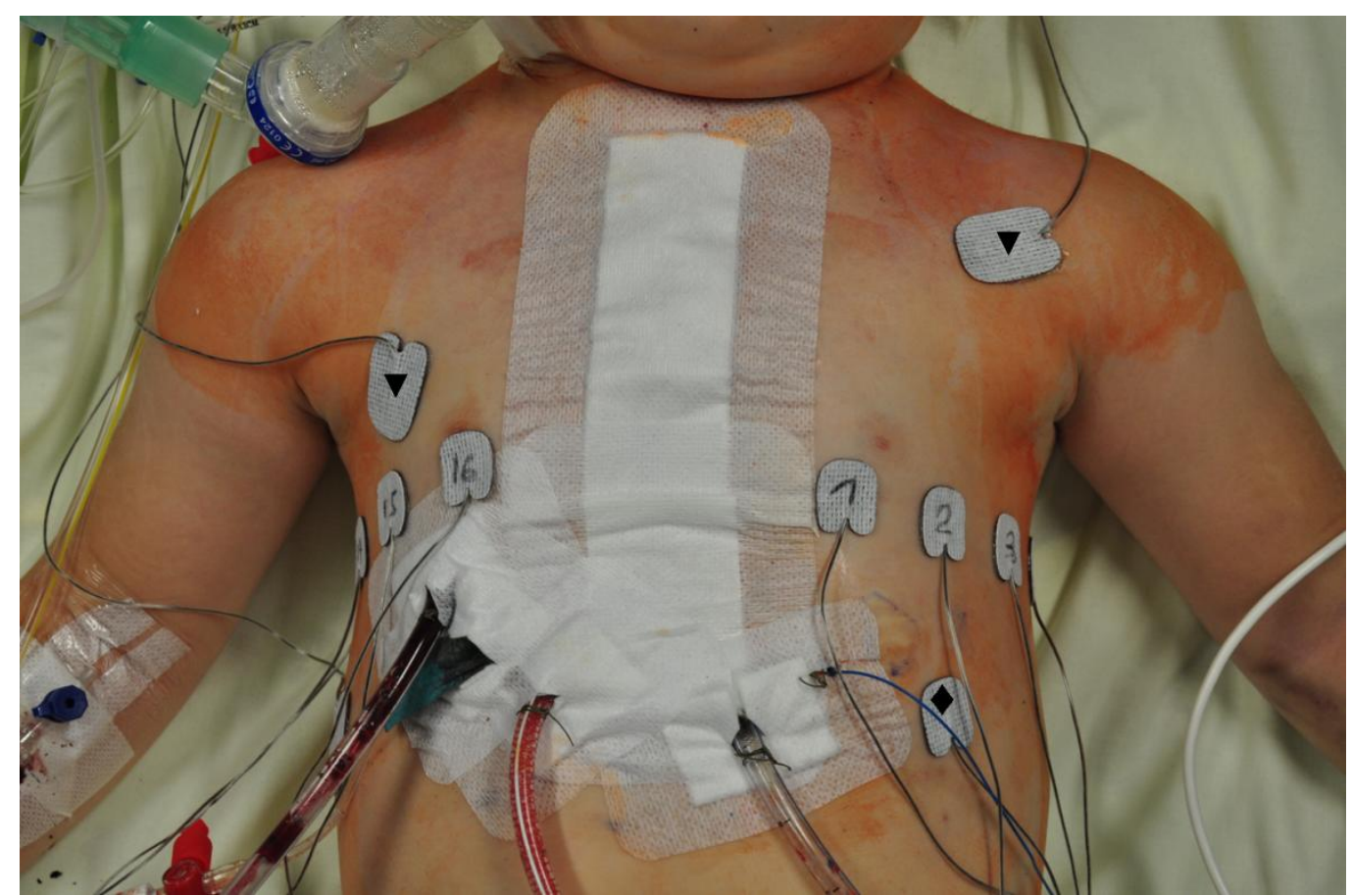

Abbildung 1b: EKG-Elektroden für die EIT-Messungen am Thorax eines Kindes auf der Intensivstation (Patient 21, Z.n. ASD-Verschluss). Referenzelektrode (\$), Elektroden zur EKG-Ableitung $(\boldsymbol{\nabla})$. 
Die zeitliche Variation der lokalen Impedanzverteilung im Thorax steht in engem Zusammenhang mit den in der Lunge herrschenden Ventilationsverhältnissen [Adler et al. 1997, Frerichs et al. 2002, Hahn et al. 1995, Harris et al. 1987, Hinz et al. 2003a, 2003b, Serrano et al. 2002]: Bei Inspiration nimmt die Impedanz in der Lunge zu, während sie bei Exspiration abnimmt. Die vermehrte Dehnung des Lungengewebes bei Inspiration führt zu kleineren Querschnitten des zwischen den Alveolen liegenden elektrisch leitenden Gewebes und damit zu höheren elektrischen Widerständen (siehe Einleitung, 1.3).

Aus den an der Thoraxoberfläche gemessenen Spannungswerten wird die Verteilung der Impedanz im Thorax berechnet und zur Bildgenerierung genutzt. Der Prozess der Generierung eines Impedanztomogramms wird als tomographische Bildrekonstruktion bezeichnet. Der dafür verwendete "Backprojection-Algorithmus“ [Barber 1990, Barber und Brown 1984, Hahn et al. 1998] liefert die relative Änderung der Impedanzverteilung bezüglich eines Referenzzustandes in Form eines zweidimensionalen Querschnittsbildes (Tomogramm). Dieses besteht aus 912 Werten, die in einer Matrix von 32 × 32 Pixeln angeordnet sind (siehe Abb. 2).

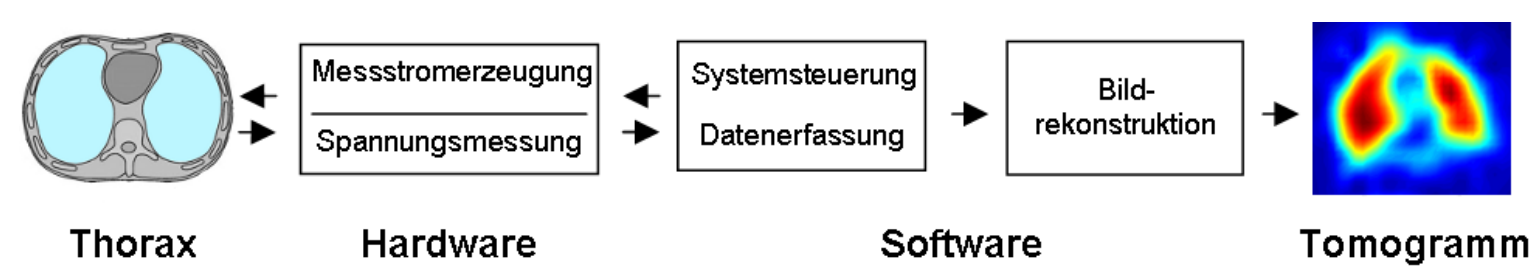

Abbildung 2: Schematische Darstellung des Grundprinzips der EIT.

Aus den bei einer Messung entstehenden einzelnen Tomogrammen wird schließlich ein Funktionsbild errechnet, das zur Darstellung der Ventilationsverteilung über den Messzeitraum (60 Sekunden) dient und der Standardabweichung der relativen Impedanzänderung der einzelnen Tomogramme entspricht (siehe Abb. 3). 


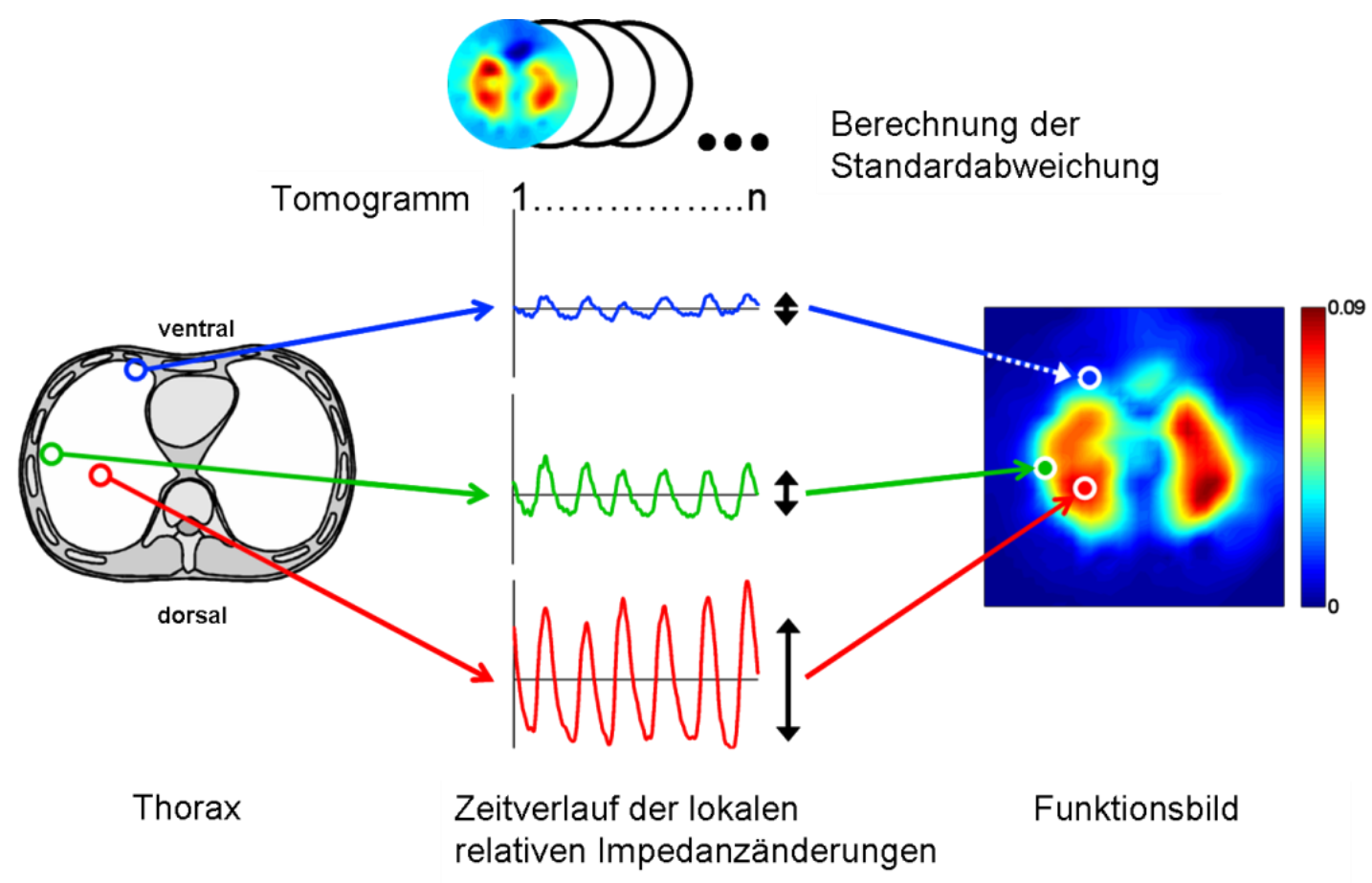

Abbildung 3: Schematische Darstellung der Entstehung eines Funktionsbildes.
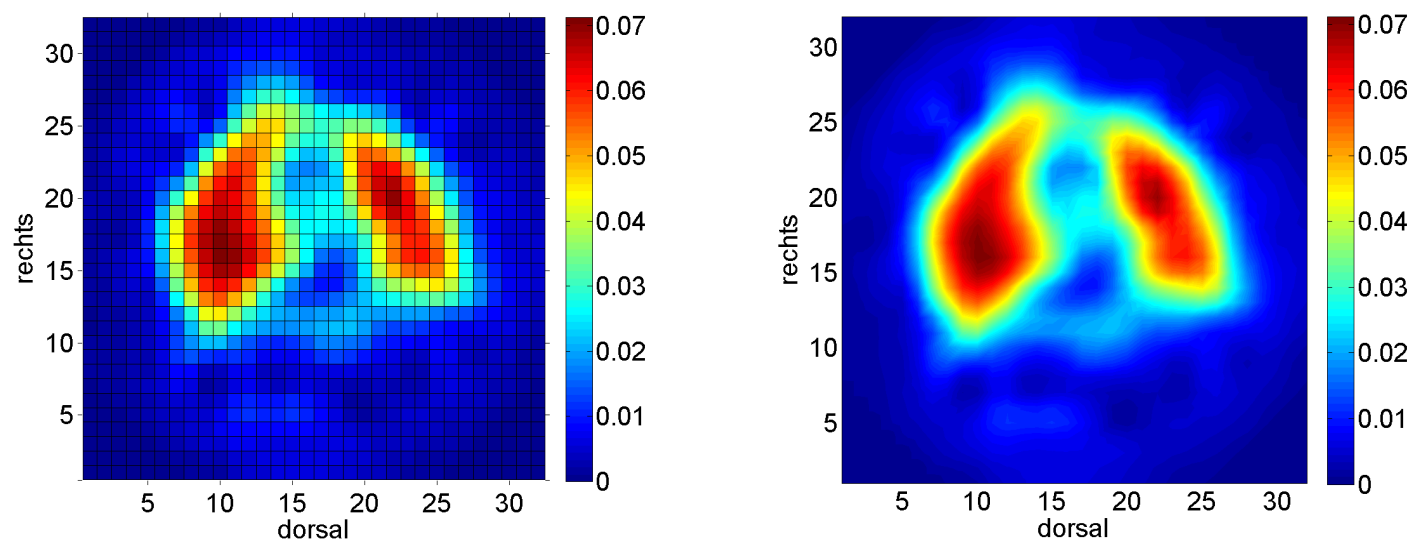

Abbildung 4: Funktionsbild der Lunge berechnet aus den einzelnen Tomogrammen über den Messzeitraum von 60 Sekunden. Im linken Bild Darstellung der einzelnen 32 x 32 Pixel. Ein einzelner Pixelwert im Funktionsbild entspricht der Standardabweichung der Werte der relativen Impedanzänderung über 60 Sekunden gemessen an diesem Ort. Regionen mit hoher Standardabweichung (rot dargestellt) entsprechen einer hohen Ventilation, Regionen mit niedriger Standardabweichung (blau dargestellt) einer niedrigen Ventilation. Die Summe aller Pixelwerte eines Funktionsbildes ist ein Maß für die Gesamtventilation. 
Bei Betrachtung der Tomogramme sowie der Funktionsbilder bei einem Thoraxschnittbild gilt dieselbe Zuordnung zu den Hauptachsen des Körpers wie bei CT- und MRT-Darstellungen: Die ventrale Seite des Thorax wird oben auf dem Bild abgebildet, während die dorsale Seite des Thorax unten auf der Tomographie zu sehen ist. Die rechte Thoraxhälfte wird auf der linken Seite im Bild dargestellt und die linke Thoraxhälfte auf der rechten Seite (siehe Abb. 4).

In dieser Arbeit wurde für alle Messungen ein EIT-Messgerät vom Typ Goe-MF II, EIT-Group Göttingen (Viasys Healthcare GmbH, Höchberg, Deutschland) eingesetzt, an dem das Messprinzip der EIT im Folgenden genauer erklärt werden soll. Dieses Hochleistungs-EIT-System ist im Vergleich zu den älteren analogen EIT-Geräten weniger störanfällig und zeichnet sich durch ein sehr gutes Signal-zu-RauschVerhältnis sowie eine große Unempfindlichkeit gegenüber elektrischen Interferenzen aus und ist damit speziell für die klinische Anwendung auf Intensivstationen konzipiert und geeignet [Hahn et al. 2001].

Das Gerät entspricht der deutschen Norm für medizinische Geräte (DIN VDE 0750 Teil 1 „Medizinische elektrische Geräte - allgemeine Festlegungen für die Sicherheit") und besitzt eine CE-Kennzeichnung.

Das EIT-System besteht aus der Hardware und der auf einem Notebook installierten Software zur Steuerung der Messvorgänge (siehe Abb. 2).

Das eigentliche EIT-Gerät (Hardware) dient zur Generierung und Einspeisung des Messstromes über die Elektrodenkabel und die 16 geklebten Oberflächenelektroden in den Thorax des Patienten. Außerdem werden die resultierenden Spannungsdifferenzen über dem Thorax ebenfalls durch das EIT-Gerät gemessen.

Die Speicherung der Daten erfolgte mittels eines handelsüblichen Notebooks (Hewlett-Packard $\mathrm{GmbH}$, Böblingen, Deutschland) mit der darauf installierten EITSoftware SCIEITV8 (Science EIT Software University of Göttingen, Version 1.02). Durch diese Software wird der Ablauf des Messvorgangs gesteuert, der das zeitliche und räumliche Muster der Stromeinspeisung und der Spannungsmessung beinhaltet. Zudem dient sie der Datenspeicherung, der online und offline verlaufenden Bildrekonstruktion sowie letztendlich auch der Weiterverarbeitung der gewonnenen EIT-Daten.

Das EIT-Gerät, der Rechner mit der Software sowie die 16 Messelektroden auf dem Thorax des Patienten bilden das als Einheit funktionierende EIT-System. 
16 handelsübliche strahlendurchlässige EKG-Elektroden (Blue Sensor BR-50-K, Medicotest, Olstykke, Dänemark) wurden zirkulär in einer Ebene um den Thorax geklebt, über die dann die Stromeinspeisung und die Potentialdifferenzmessungen erfolgte. Die Verträglichkeit und elektrischen Eigenschaften der Elektroden wurden durch Baisch et al. (1995) in Langzeitmessungen über 24 Stunden geprüft. Zur Datengewinnung wurde ein geringer Wechselstrom (5 mA, $100 \mathrm{kHz}$ ) zwischen zwei jeweils aneinander angrenzende Elektroden eingespeist. Die resultierenden Oberflächenpotentiale wurden zwischen den restlichen 13 nicht an der Stromeinspeisung beteiligten Elektrodenpaaren gemessen. Da das Gewebe elektrisch leitfähig ist, kommt es durch den Stromfluss zu einer bestimmten Verteilung der elektrischen Äquipotentiallinien innerhalb der durch die Elektroden definierten Ebene [Hahn et al. 1998]. Die Endpunkte der Linienbündel treffen mit dem Anbringungsort der verbleibenden Elektroden zusammen, so dass die Potentialdifferenzen zwischen diesen Linienbündeln näherungsweise bestimmt werden. Alle 16 Elektrodenpaare wurden der Reihe nach als aktive Einspeiselektroden genutzt, und das oberflächliche Potential wurde zwischen den restlichen passiven 13 Elektrodenpaaren gemessen. Ein Messzyklus war beendet, wenn alle Elektrodenpaare einmal als „Einspeiselektroden“ gedient haben. Somit gilt für das 16-Elektroden-System, dass ein kompletter Messzyklus $16 \times 13$, also 208 Messwerte liefert, aus denen ein Tomogramm erzeugt wird. Als "frame rate" (Bildfrequenz) wird die Anzahl der kompletten Messzyklen im Zeitraum von einer Sekunde bezeichnet. Für die Messung mit 16 Elektroden wurde eine frame rate (Bildfrequenz) von $13 \mathrm{~Hz}$ eingestellt, d.h. dass $13 \mathrm{Mal}$ pro Sekunde ein kompletter Messzyklus ablief. Eine vollständige Messung am Krankenbett dauerte in dieser Arbeit 60 Sekunden, so dass in dieser Zeit pro Messung 162.240 Messwerte erhoben wurden, aus denen ein Funktionsbild errechnet wurde.

\subsection{Versuchsdurchführung}

Für die EIT-Messung wurden bei den in die Studie aufgenommenen Patienten nach Übernahme auf die pädiatrische Intensivstation 16 konventionelle röntgentransparente EKG-Klebeelektroden (siehe 2.3) $1-2 \mathrm{~cm}$ unterhalb der Mamillarebene in einer Ebene zirkulär um den Thorax geklebt (siehe Abb. 1). Die Elektroden für die Neugeborenen und Säuglinge mussten aufgrund der kleinen 
anatomischen Verhältnisse auf dem Thorax durch Zuschneiden der Patientengröße angepasst werden, wie schon bei Hampshire et al. (1995) beschrieben. Das Kleben wurde mit der ersten Elektrode auf der linken Thoraxhälfte möglichst direkt über dem Sternum begonnen, was aufgrund des Wundverbands über der medianen Sternotomie nicht immer exakt praktikabel war. Dann wurden die übrigen Elektroden in gleichen Abständen zirkulär um den Thorax platziert. Im Bereich des Epigastriums wurde eine weitere Elektrode als Referenzelektrode aufgeklebt (siehe Abb. 1).

Die Elektroden wurden schließlich an das EIT-Gerät angeschlossen und es erfolgte die Messung der elektrischen Potentialdifferenzen mittels des mit der speziellen Software (SCIEITV8, siehe 2.3) ausgerüsteten Notebooks.

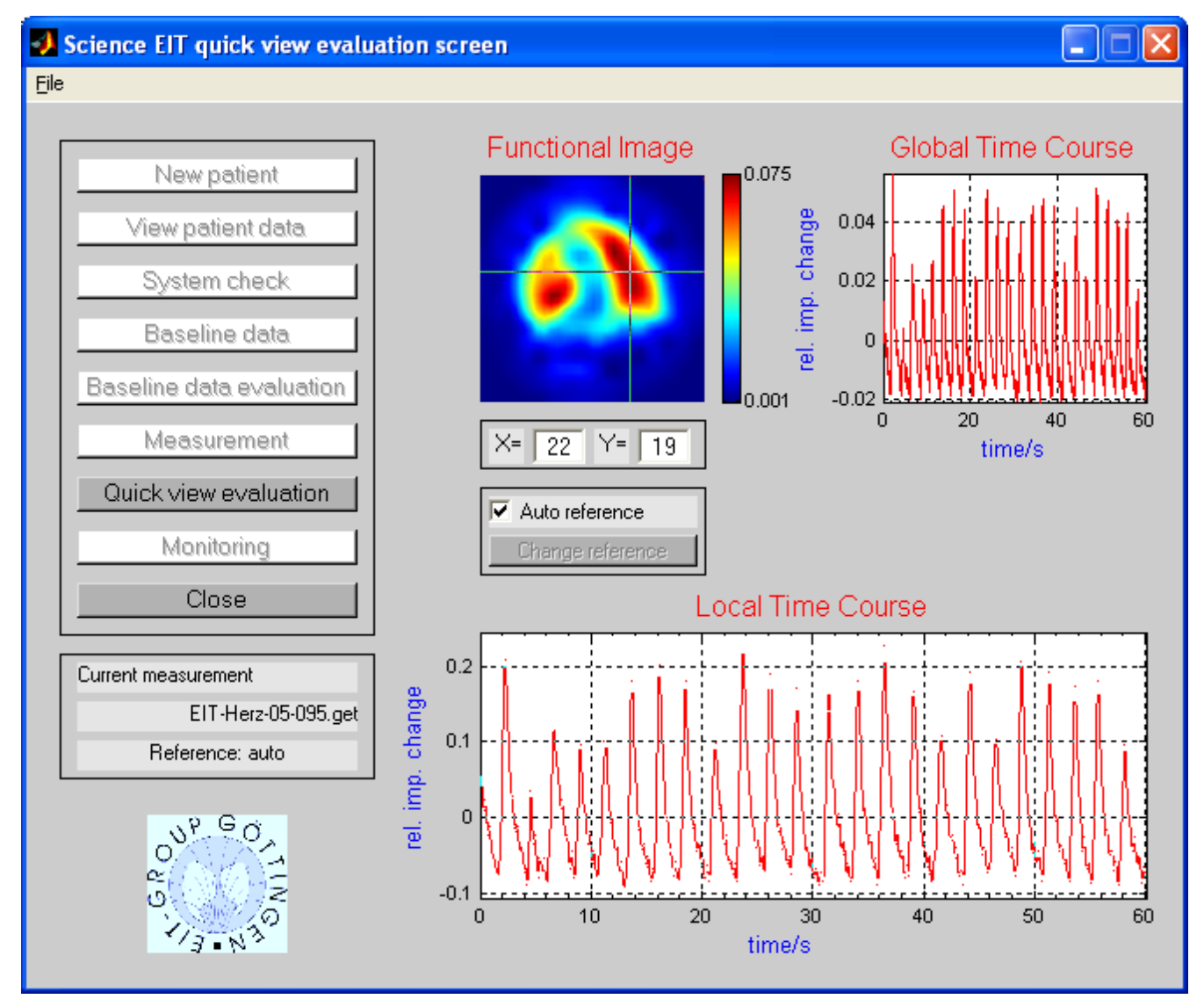

Abbildung 5: Screenshot der Messsoftware des Göttinger EIT-Systems. Nach einer Messung über 60 Sekunden Darstellung des Funktionsbildes (Functional Image) sowie des globalen Zeitverlaufes (Global Time Course) der relativen Impedanzänderung und des lokalen Zeitverlaufes (Local Time Course) an einem bestimmten beliebig auswählbaren Ort im Funktionsbild. Anhand der Ausschläge der relativen Impedanzänderung im globalen und lokalen Zeitverlauf lässt sich die Atemfrequenz des Patienten auszählen. 
Die Messungen wurden diskontinuierlich für die Dauer von jeweils 60 Sekunden durchgeführt. Die EIT-Daten der einzelnen Messungen wurden während der Datenerfassung unter individuellen Dateinamen anonymisiert auf der Festplatte des Notebooks gespeichert und gleichzeitig in einem Protokoll dokumentiert. In diesem Protokoll wurden außerdem noch weitere für die Studie relevante MonitoringParameter erfasst (siehe Tab. 2):

- Datum, Uhrzeit der Messung,

- Beatmungsmodus (SIMV, BIPAP, CPAP),

- Inspirationsdruck ( $\left.P_{\text {insp }}\right)$,

- Inspirationszeit ( $\left.T_{\text {insp }}\right)$,

- positiver endexspiratorischer Druck (PEEP),

- Atemminutenvolumen (AMV),

- Atemfrequenz (AF),

- Sauerstoffraktion im eingeatmeten Gasgemisch $\left(\mathrm{FiO}_{2}\right)$,

- transkutane Sauerstoffsättigung $\left(\mathrm{S}_{\mathrm{a}} \mathrm{O}_{2}\right)$,

- Sauerstoffpartialdruck $\left(\mathrm{p}_{\mathrm{a}} \mathrm{O}_{2}\right)$,

- Kohlendioxidpartialdruck $\left(\mathrm{p}_{\mathrm{a}} \mathrm{CO}_{2}\right)$.

Die Messungen wurden, soweit es der klinische Verlauf zuließ, nach einem definierten Plan angefertigt: Nach Übernahme aus dem OP (meist früher Nachmittag) wurden stündliche Messungen durchgeführt. Zusätzlich erfolgten Messungen 20 Minuten nach Veränderungen der Beatmungsparameter sowie wenige Minuten vor der Extubation. Die Messungen nach der Extubation fanden für die ersten 30 Minuten in 10-minütigem Abstand statt, anschließend halbstündlich bis stündlich. Die Datenerfassung wurde beendet, wenn sich etwa 1-4 Stunden nach Extubation stabile respiratorische Verhältnisse (Eupnoe, ausgeglichene Blutgasanalyse) eingestellt hatten.

In dieser Studie stellte die Extubation für die Datengewinnung ein wichtiges Ereignis dar, da die Patienten nach der Entfernung des Tubus wieder ohne maschinelle Hilfe selbstständig atmen mussten. Die Auswirkungen dieses Effektes auf die regionalen Ventilationsverhältnisse sollten durch EIT-Messungen kurz vor und kurz nach Extubation dargestellt werden.

Die Extubation stellte für die Patienten eine Stresssituation dar und sie mussten sich zunächst an die „selbstständige“ Atmung gewöhnen. Zur Vermeidung von Störungen 
bei der Datenerhebung, wie z.B. Husten, Weinen oder Bewegungen des Patienten, wurde erst 10 Minuten nach der Extubation, wenn sich erfahrungsgemäß ein erstes Gleichgewicht eingestellt hat, erneut gemessen.

Wenn der Patient allerdings über Nacht oder über mehrere Tage maschinell weiter beatmet wurde, wurden die Abstände der einzelnen Messungen auf 4-6 Stunden ausgedehnt. Bei diesen Patienten war es teilweise erforderlich, die durch Schwitzen, Bewegungen, Waschen und Umlagern nicht mehr gut haftenden Elektroden an gleicher Thoraxstelle neu zu kleben, was keinen signifikanten Einfluss auf die Impedanzmessungen hat [Frerichs et al. 1998a, Marven et al. 1996, Reifferscheid et al. 2011, Victorino et al. 2004].

Die Gesamtzahl der bei einem einzelnen Patienten durchgeführten Messungen sowie die Zeitabstände zwischen den einzelnen Messungen hingen letztendlich von der Dauer und dem Verlauf der postoperativen maschinellen Beatmung sowie von therapeutischen Interventionen ab und waren somit individuell verschieden.

\subsection{Versuchsdokumentation}

Für jeden Patienten wurde während der Datenerhebung ein Versuchsprotokoll angefertigt, in dem jede EIT-Messung mit den Werten der zu dem jeweiligen Zeitpunkt erhobenen Routineparameter dokumentiert wurde (siehe Tab. 2). Im Kopf des Protokolls wurden außerdem das Geburtsdatum, das aktuelle Gewicht und die Größe des Patienten sowie die Art des Herzfehlers und die erfolgte Herzoperation festgehalten. Auch das verwendete Beatmungsgerät (siehe 2.2.4) und der Beatmungsmodus wurden erfasst.

Tabelle 2: Versuchsprotokoll.

\begin{tabular}{|c|c|c|c|c|c|c|c|c|c|c|}
\hline $\begin{array}{l}\text { Messung } \\
\text { Datum/Zeit }\end{array}$ & $\begin{array}{c}\mathrm{S}_{\mathrm{a}} \mathrm{O}_{2} \\
\%\end{array}$ & $\begin{array}{l}\mathbf{P}_{\text {insp }} \\
\text { mbar }\end{array}$ & $\begin{array}{l}T_{\text {insp }} \\
\text { sec }\end{array}$ & $\begin{array}{c}\text { PEEP } \\
\text { mbar }\end{array}$ & $\begin{array}{l}\mathbf{A F} \\
/ \mathrm{min}\end{array}$ & $\begin{array}{l}\text { AMV } \\
\text { //min }\end{array}$ & $\underset{\%}{\mathrm{FiO}_{2}}$ & $\begin{array}{l}\mathbf{p}_{\mathrm{a}} \mathrm{CO}_{2} \\
\mathrm{mmHg}\end{array}$ & $\begin{array}{l}\mathrm{paO}_{2} \mathrm{O}_{2} \\
\mathrm{mmHg}\end{array}$ & $\begin{array}{l}\text { Son stige s } \\
\text { Kopflage, } \\
\text { Eektroden, etc }\end{array}$ \\
\hline
\end{tabular}

Die aktuelle transkutane Sauerstoffsättigung $\left(\mathrm{S}_{\mathrm{a}} \mathrm{O}_{2}\right)$ wurde kontinuierlich im Rahmen des Routinemonitorings über ein Pulsoxymeter gemessen und konnte zum Zeitpunkt der EIT-Messung vom Überwachungsmonitor (Intellivue, Philips Deutschland GmbH, Hamburg, Deutschland) abgelesen werden. Die Werte des $\mathrm{O}_{2^{-}}$und $\mathrm{CO}_{2^{-}}$ 
Partialdrucks stammten aus den arteriellen oder venösen Blutgasanalysen. Erstere lagen regelmäßig gemessen nur von 23 Patienten vor, da nicht alle Patienten über einen gut rückläufigen arteriellen Zugang verfügten und die Werte daher venös gemessen wurden. Von dem Beatmungsgerät wurden die in dem Protokoll aufgeführten Beatmungsparameter wie $\mathrm{P}_{\text {insp, }}, T_{\text {insp }}, \mathrm{PEEP}, \mathrm{AF}, \mathrm{AMV}$ und $\mathrm{FiO}_{2}$ parallel zu jeder EIT-Messung abgelesen.

\subsection{Auswertung der Daten}

Während der Datenaufnahme wurden die Spannungsmesswerte von der EITHardware auf den damit verbundenen PC übertragen und dort von der Messsoftware gespeichert. Die Rekonstruktion der Tomogramme erfolgte anschließend offline. Ein etablierter Parameter für die Stärke der Ventilation an einem bestimmten Ort (Pixel) des durch ein $32 \times 32$-Gitter definierten Thoraxquerschnitts ist die Standardabweichung der zeitlichen Abfolge der Werte der Tomogramme an diesem Ort. Um beispielsweise den Pixelwert an der Koordinate $(x, y)$ zu erhalten, wird die Standardabweichung über die Pixelwerte aller 780 Tomogramme einer Messung (bei einer Bildfrequenz von $13 \mathrm{~Hz}$ und einer Aufzeichnungsdauer von 60 Sekunden) am Ort $(x, y)$ berechnet. Aus allen Einzeltomogrammen entsteht somit ein Funktionsbild (siehe Abb. 3), das aus einer einzigen $32 \times 32$-Pixel-Matrix besteht und die Standardabweichung der relativen Impedanzänderung aller Tomogramme darstellt [Hahn et al. 1995].

Zunächst wurden die Daten auf ihre Verwertbarkeit geprüft, indem auf Artefakte innerhalb einer Messung geachtet wurde. Vollständig gestörte Messdaten wurden komplett verworfen, während aus nur teilweise gestörten Messdaten die nicht auswertbaren Bereiche entfernt wurden und dann lediglich die selektierte Datensequenz zur Auswertung herangezogen wurde.

Zur vereinfachten Darstellung der Ventilationsverteilung über den gesamten Zeitraum der Datengewinnung eines Patienten wurden mit Hilfe der Software MATLAB (Version 7,0, 2004, The Math Works Inc. Germany, Ismaning, Deutschland) Profilkurven erstellt, die die Ventilationssituation der Lunge einmal von dorsal nach ventral und einmal von rechts nach links darstellen (siehe Abb. 6).

Zur Gewinnung des Rechts-Links-Profils wurden die Werte des Funktionsbildes in dorsal-ventral-Richtung spaltenweise aufaddiert, während die Werte zur Gewinnung 
des Dorsal-Ventral-Profils zeilenweise von rechts nach links aufaddiert wurden (siehe Abb. 6). Als Profilwert wird die Summe der Pixelwerte des Funktionsbildes einer Spalte/ Reihe bezeichnet; dieser stellt die Summe der Standardabweichungen der relativen Impedanzänderung einer Spalte/ Reihe über den Messzeitraum von 60 Sekunden dar. Analog zu der in der EIT-Literatur üblichen Nomenklatur wird in der vorliegenden Arbeit auch diese Summe der Standardabweichungen der relativen Impedanzänderung grundsätzlich als relative Impedanzänderung (rel. $\Delta Z$ ) bezeichnet. Die Profilwerte des Rechts-Links-Profils sind somit ein Maß für die Verteilung der Gesamtventilation in Rechts-Links-Richtung des entsprechenden Funktionsbildes, wohingegen die Profilwerte des Dorsal-Ventral-Profils der Verteilung der Gesamtventilation in Dorsal-Ventral-Richtung entsprechen (siehe Abb. 6).

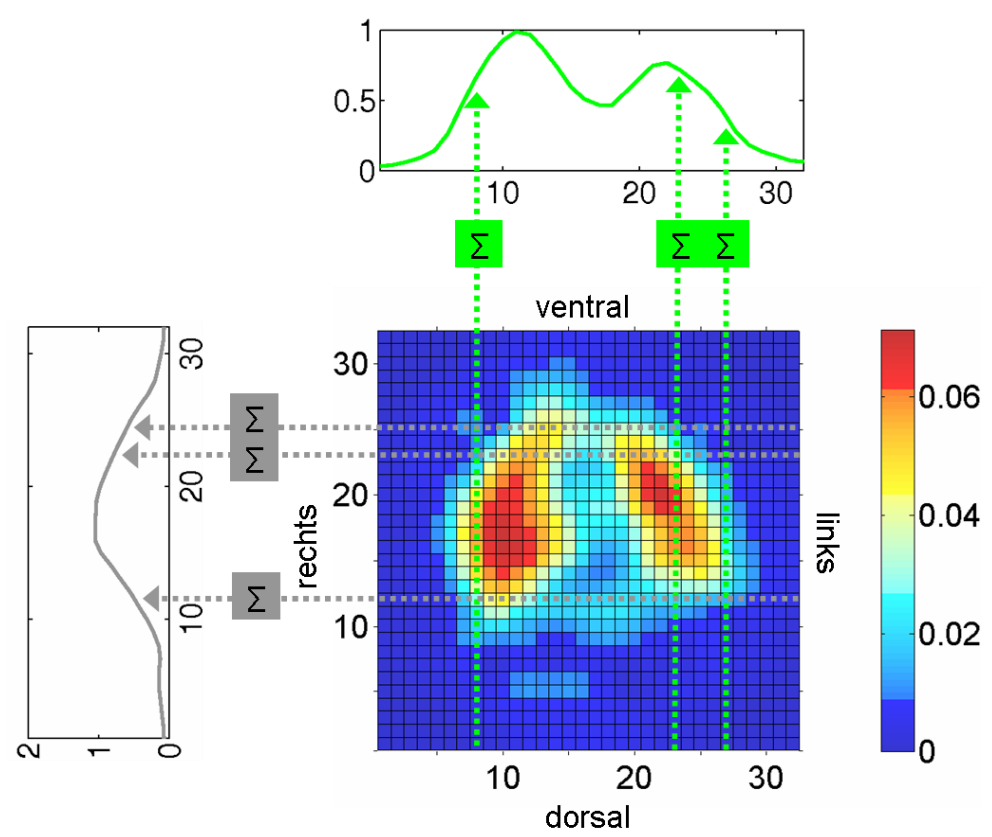

Abbildung 6: Schematische Darstellung der Entstehung der Profilkurven (grau für dorsal-ventral, grün für rechts-links) aus dem Funktionsbild einer Messung (60 Sekunden). Für das Dorsal-Ventral-Profil erfolgt das Aufsummieren der Werte jeweils einer der 32-Pixel-Reihen von links nach rechts; für das Rechts-Links-Profil erfolgt das Aufsummieren der Werte jeweils einer der 32-Pixel-Reihen von dorsal nach ventral.

Um schließlich die Veränderung der Ventilationsverteilung über den gesamten Behandlungszeitraum sichtbar zu machen, wurde der Verlauf der verschiedenen Profilkurven einer Messreihe über der Zeit als Fläche dargestellt (siehe Abb. 7, 8, 9). 

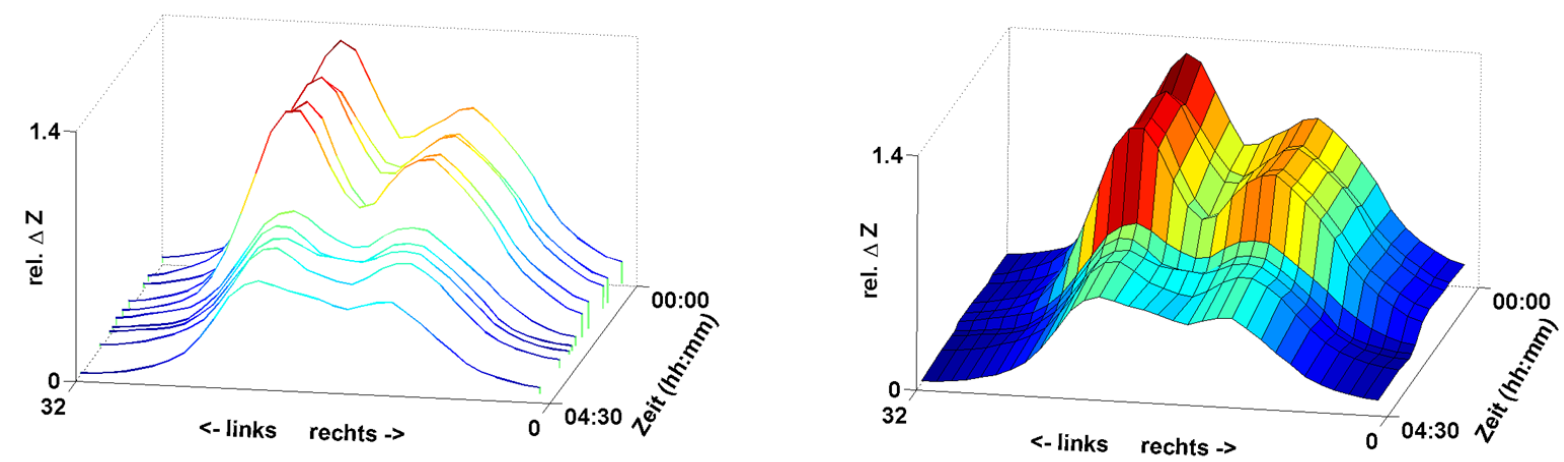

Abbildung 7: Links Darstellung der einzelnen Profilkurven einer Messreihe, rechts Darstellung der Profilkurven als Fläche, rel. $\Delta Z$ = relative Impedanzänderung.

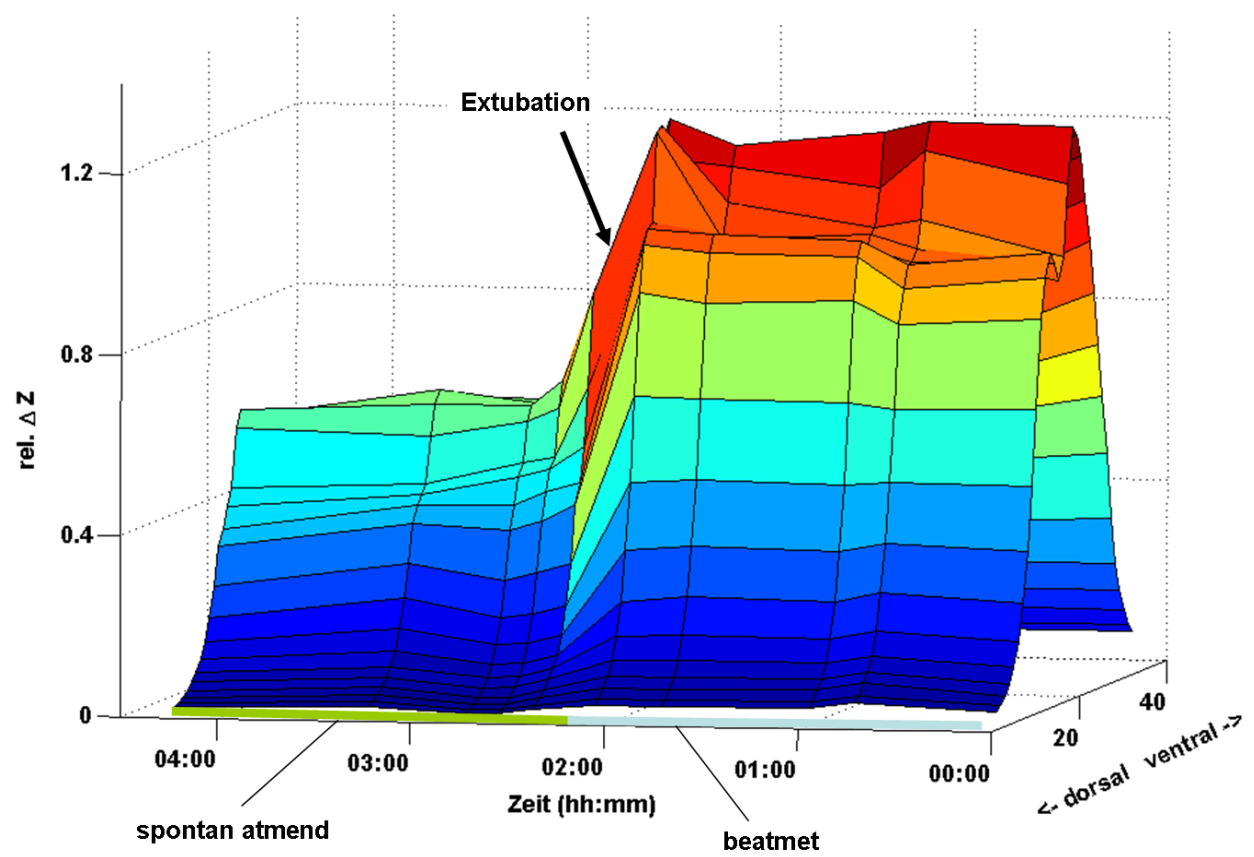

Abbildung 8: Darstellung der einzelnen Profilkurven - als Fläche verbunden - als Profilverlauf über den gesamten Messzeitraum von Patient 24 für den ventralen und dorsalen Lungenbereich. Von rechts nach links verlaufende Zeitachse, um den dorsalen Lungenbereich besser darstellen zu können. 


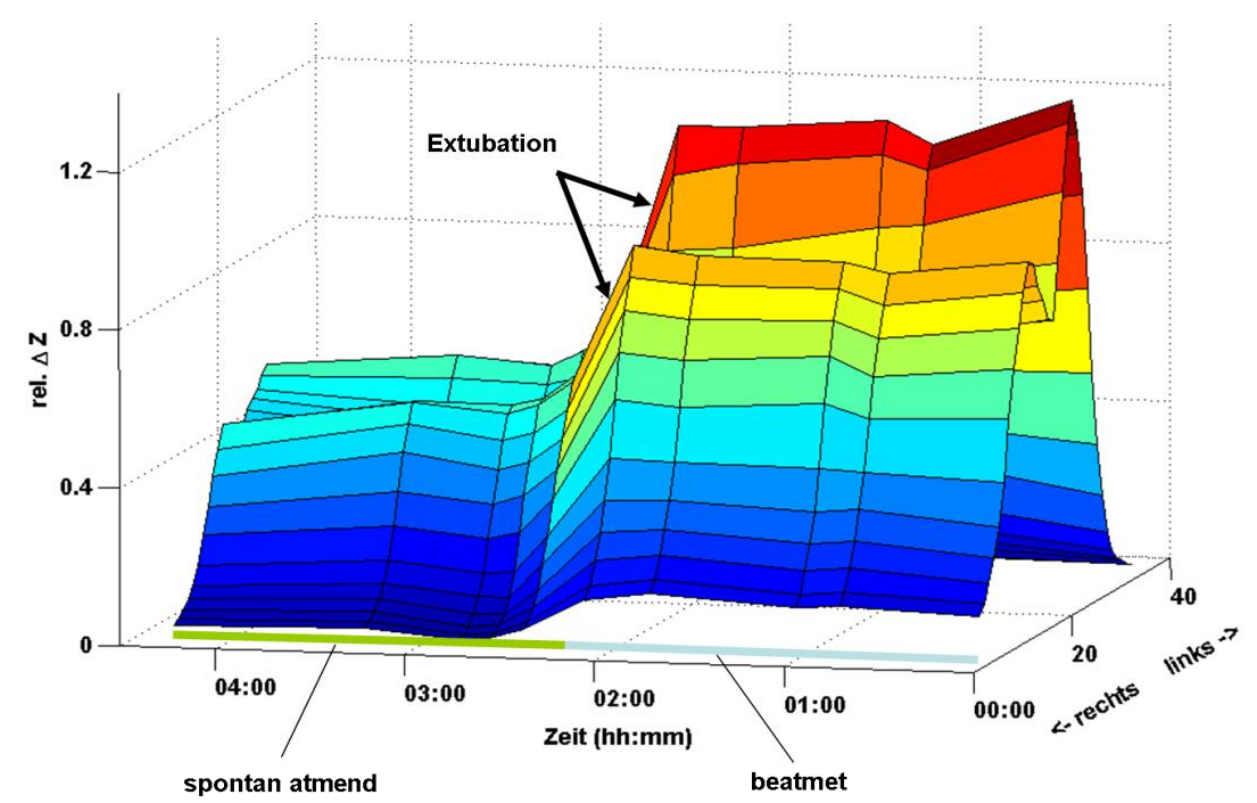

Abbildung 9: Darstellung der einzelnen Profilkurven - als Fläche verbunden - als Profilverlauf über den gesamten Messzeitraum von Patient 24 für den rechten und linken Lungenflügel. Von rechts nach links verlaufende Zeitachse, um den linken Lungenflügel besser darstellen zu können.

Mit Hilfe einer spezifischen Software (Eigenprogrammierung EIT Group Göttingen, Dittmar et al. 2010) konnten die durch die Messungen gewonnenen Daten der Funktionsbilder bearbeitet werden. Innerhalb des Funktionsbildes wurden verschiedene Bereiche (Regions of Interest) festgelegt: Der ventrale und dorsale Bereich der Messebene, der rechte und linke Bereich sowie der Gesamtbereich. Somit konnte schließlich die relative Impedanzänderung und damit die Ventilationsverteilung in den festgelegten Bereichen gesondert und vergleichend betrachtet werden. Nach Übertragung der Zahlenwerte der verschiedenen Bereiche in Excel (Office 2003, Microsoft Deutschland GmbH, Unterschleißheim, Deutschland) wurden anschließend die Summen der relativen Impedanzänderung für den gesamten thorakalen Bereich (siehe Abb. 10) sowie für die Bereiche rechts/links und ventral/ dorsal (siehe Abb. 11) berechnet und als Verlaufskurven graphisch gegeneinander aufgetragen. 


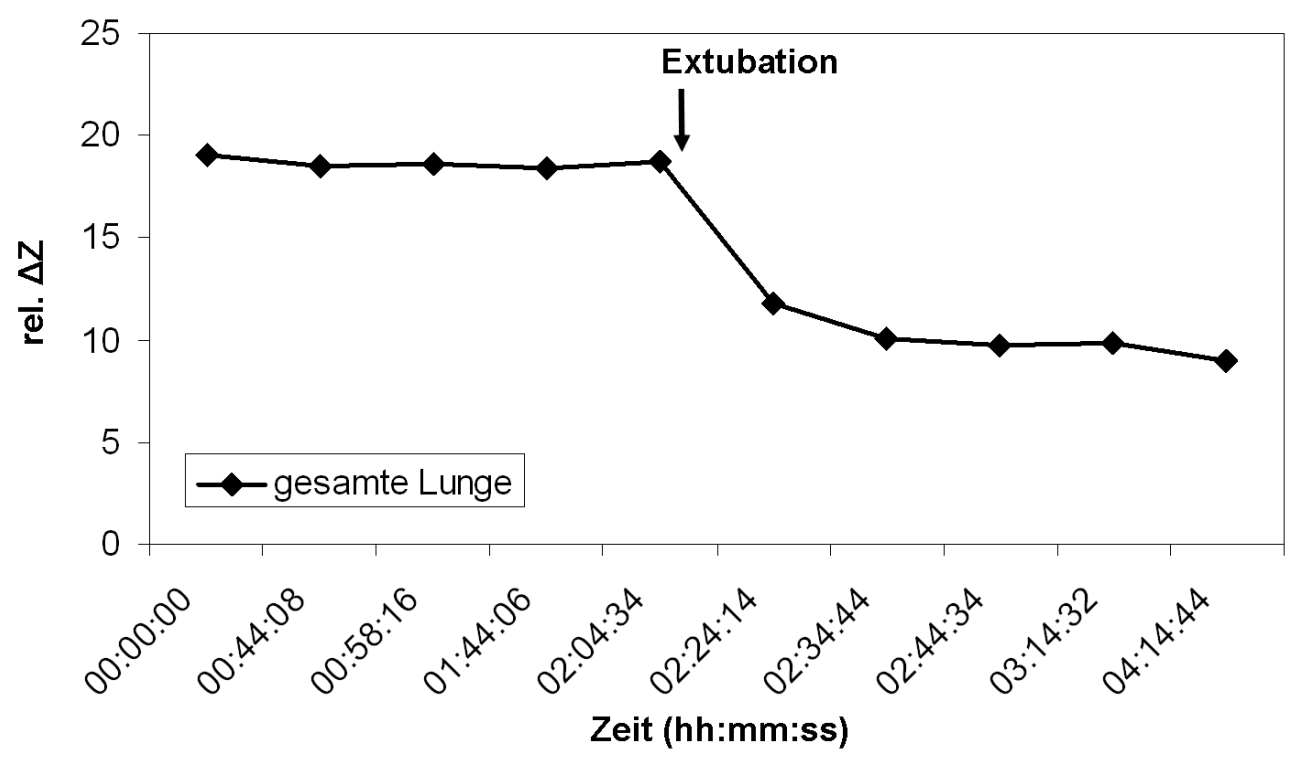

Abbildung 10: Verlauf der Summen der relativen Impedanzänderung (rel. $\Delta Z$ ) der gesamten Lunge über den Messzeitraum von Patient 24. Nach der Extubation ist ein deutlicher Abfall der relativen Impedanzänderung zu sehen.
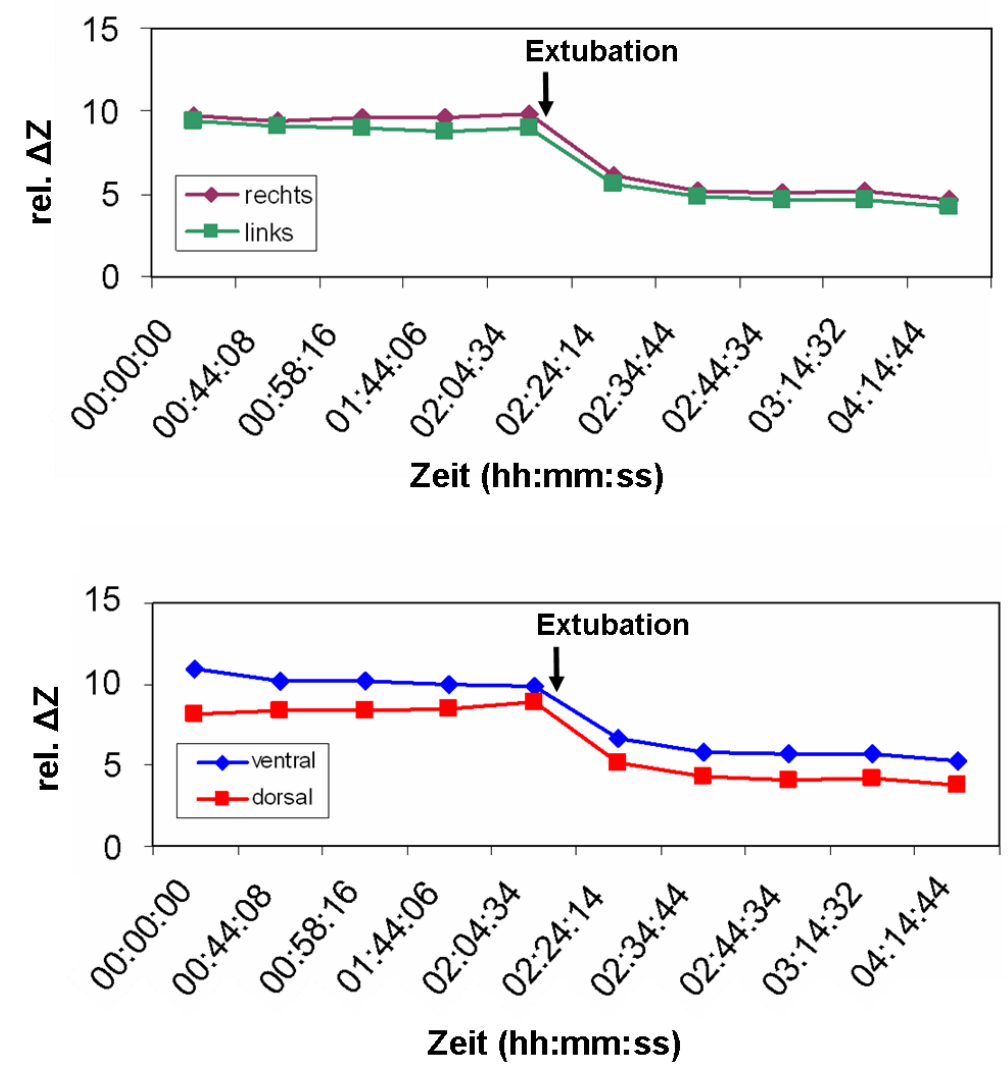

Abbildung 11: Verlauf der Summen der relativen Impedanzänderung (rel. $\Delta Z$ ) des rechten und linken Lungenflügels sowie der ventralen und dorsalen Lunge über den Messzeitraum von Patient 24. Nach der Extubation ist ein deutlicher Abfall der relativen Impedanzänderung zu sehen. 


\subsection{Statistische Analyse}

Die Ergebnisse wurden, wenn nicht anders gekennzeichnet, als Mittelwert \pm Standardabweichung, in den Grafiken mit dem $95 \%$ Konfidenzi ntervall angegeben. Teilweise wurde auch der Variationskoeffizient verwendet. Für die statistischen Analysen wurden die Daten auf Normalverteilung und Varianzhomogenität geprüft, bevor die je nach Voraussetzung adäquaten statistischen Verfahren zur Anwendung kamen: Korrelation (nach Pearson $\left(r_{p}\right)$ oder Spearman $\left(r_{s}\right)$ ), t-Test für verbundene und unverbundene Stichproben, ANOVA (Analysis of Variance), Kruskal-Wallis Test bzw. mehrfaktorielle Varianzanalyse (GLM = General Linear Model). Dabei diente der partielle Eta ${ }^{2}$-Wert zur Erklärung der durch die einzeInen Variablen bedingten Varianzanteile. Die verschiedenen Messungen eines Patienten (Lungenabschnitte, verschiedene Beatmungsmodi und -parameter, vor/ nach Extubation) wurden als wiederholte Messungen behandelt und als abhängige, verbundene Stichproben getestet. Dabei kamen der t-Test für verbundene Stichproben sowie Varianzanalysen für Repeated Measurements (GLMRM = General Linear Model for Repeated Measurements) zum Einsatz, um die Bedeutung von Alter und Geschlecht als Zwischensubjektfaktoren bewerten zu können.

Alle Tests wurden mit SPSS 15.0 (IBM, Somers, USA) und zweiseitig durchgeführt, als Signifikanzgrenze wurde $p \leq 0,05$ festgelegt. Mit "marginal signifikant" wurden Ergebnisse mit $p \leq 0,1$ bewertet. 


\section{Ergebnisse}

Bei 29 von 30 untersuchten Patienten waren aussagekräftige EIT-Messungen während des Beatmungsverlaufes und nach der Extubation unter Spontanatmung reproduzierbar möglich. Die Daten eines einzigen Patienten konnten aufgrund von messtechnischen Problemen durch starke externe elektrische Störungen während des gesamten Messintervalls nicht für die Auswertung herangezogen werden; die Störungsquelle war nicht zu eruieren. Bei den übrigen 29 Patienten kam es zu keinen Interferenzen zwischen dem EIT-Gerät und den zur Patientenüberwachung sowie zur Therapie notwendigen elektrischen Geräten auf der Intensivstation. Die im Rahmen der postoperativen Versorgung notwendigen diagnostischen, therapeutischen und pflegerischen Maßnahmen wurden durch die Messungen nicht beeinflusst.

Für die graphische Darstellung der relativen Impedanzänderung und somit der Ventilationsverteilung über den gesamten Beobachtungszeitraum eines jeden Patienten wurden Profilkurven der dorsalen und ventralen Lungenabschnitte sowie des rechten und linken Lungenflügels und außerdem Verlaufskurven der Ventilation für die gesamte Lunge sowie für die genannten Lungenbereiche herangezogen (siehe Patienten und Methoden, 2.6).

\subsection{Verteilung der relativen Impedanzänderung während maschineller Beatmung und nach Extubation}

Zur Messung und Beurteilung der relativen Impedanzänderung während maschineller Beatmung und unter Spontanatmung wurden regelmäßig EIT-Messungen vor und nach der Extubation der Patienten vorgenommen (siehe Patienten und Methoden, 2.4). Bei 28 von 29 Patienten kam es unter Spontanatmung zu einer deutlichen Abnahme der relativen Impedanzänderung; die Patienten hatten im beatmeten Zustand somit eine höhere Ventilation als im spontan atmenden Zustand. Im Gegensatz zu dieser Beobachtung konnte bei einem Patienten (Patient 23) nach Extubation eine Zunahme der relativen Impedanzänderung und damit der Ventilation gemessen werden (siehe Tab. 4 und Abb. 23).

In den erstellten Funktionsbildern sowie den Verlaufs- und Profilkurven für dorsal/ ventral und rechts/ links konnte die Ventilationsverteilung unter Beatmung und unter 
Spontanatmung für jeden Patienten dargestellt werden (siehe Patienten und Methoden, 2.6). Beispielhaft soll hier der Verlauf der relativen Impedanzänderung und damit die unterschiedliche Verteilung der Ventilation für Patient 13 gezeigt werden, ein 3-jähriges Mädchen nach Verschluss eines Vorhofseptumdefektes vom Sekundumtyp.
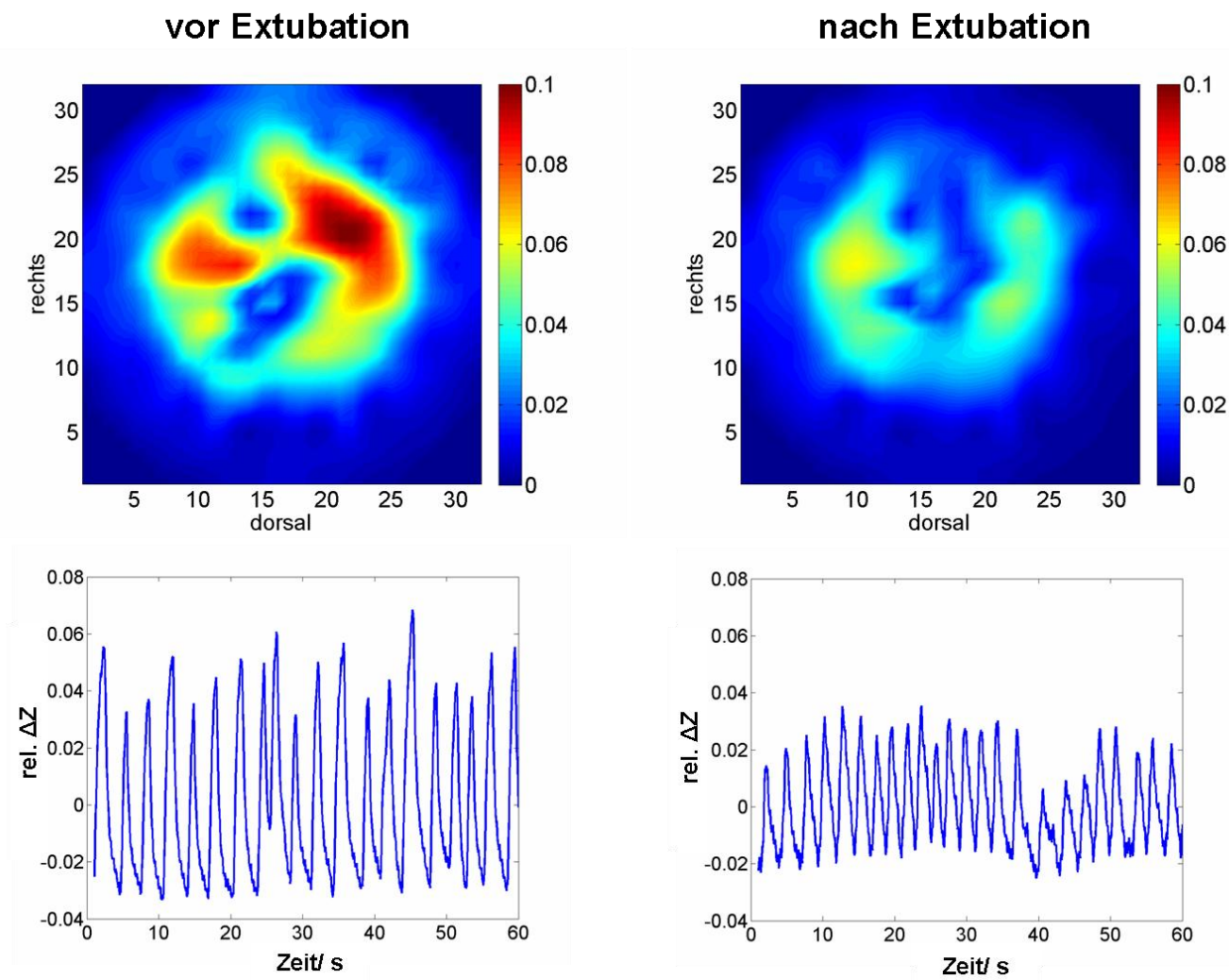

Abbildung 12: Funktionsbilder der Lunge (oben) sovie die dazugehörigen globalen Zeitverläufe (unten) unmittelbar vor und unmittelbar nach Extubation von Pat. 13. Die relative Impedanzänderung (rel. $\Delta Z$ ) nach Extubation ist deutlich geringer, die Atemfrequenz mit 24/ min nach Extubation im Vergleich zu 20/ min vor Extubation höher.

In den Funktionsbildern in Abb. 12 ist direkt vor der Extubation eine deutlich höhere relative Impedanzänderung in der Lunge zu sehen als unmittelbar nach der Extubation. Auch die globalen Zeitverläufe (siehe Abb. 12) veranschaulichen den Unterschied: In der Messung vor Extubation ist die relative Impedanzänderung über dem Thoraxquerschnitt größer, die Lunge weist durch die durch das Beatmungsgerät verursachte stärkere Dehnung des Lungengewebes höhere relative Impedanzunterschiede zwischen In- und Exspiration auf, was mit einer besseren 
Ventilation korreliert (siehe Einleitung, 1.3). Nach Extubation zeigen sich im globalen Zeitverlauf niedrigere Impedanzänderungen und eine höhere Atemfrequenz (24/ min zu vorher 20/ $\mathrm{min}$ ), die Patientin atmete schneller und flacher.

Die Werte der relativen Impedanzänderung der gesamten Lunge aufgetragen für jeden Messzeitpunkt zeigen den Beatmungs- und Spontanatmungsverlauf (siehe Abb. 13) über den gesamten Beobachtungszeitraum. Es ist ein deutlicher Abfall der relativen Impedanzänderung, entsprechend einer Abnahme der Ventilation nach Extubation zu sehen.

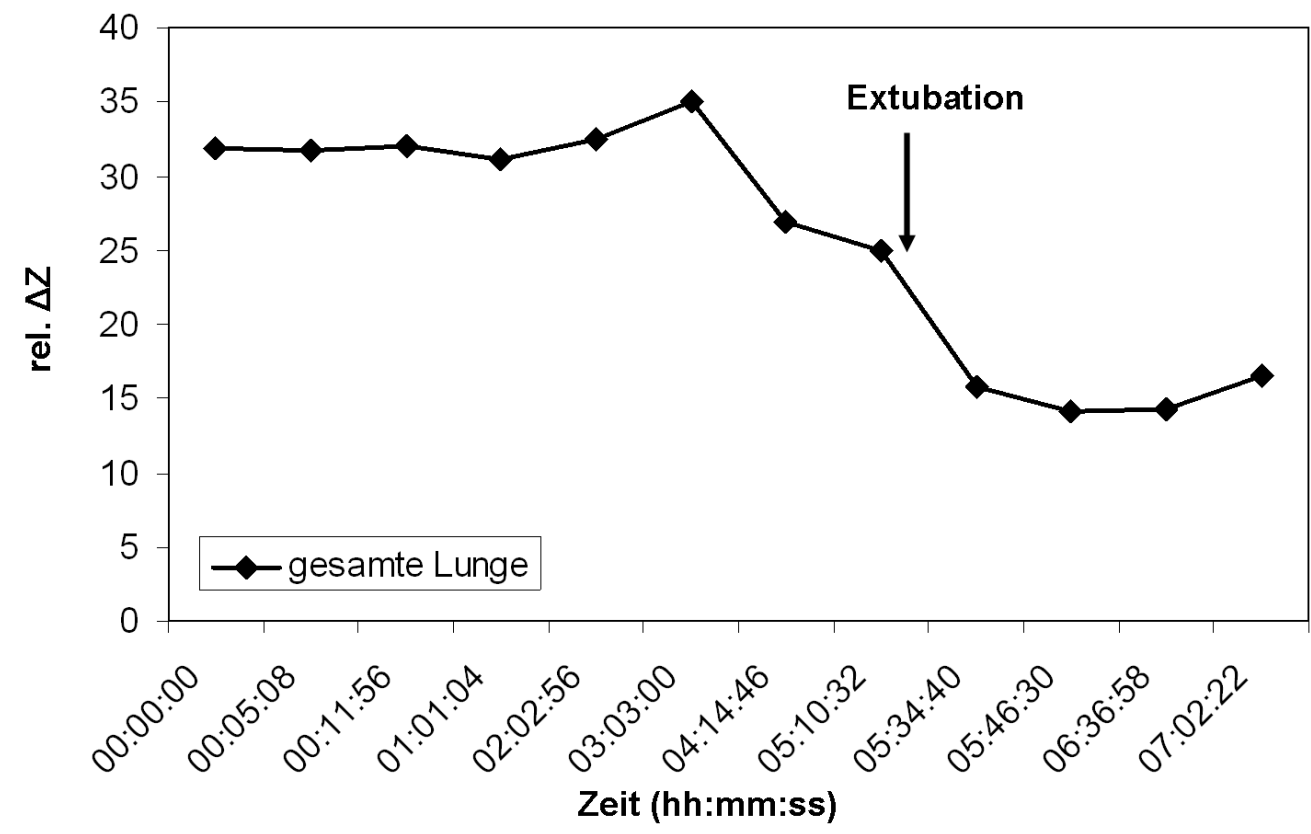

Abbildung 13: Verlauf der Summen der relativen Impedanzänderung (rel. $\Delta Z$ ) der gesamten Lunge über den Messzeitraum von Patient 13. Bereits vor Extubation kommt es aufgrund einer Reduktion der Beatmungsparameter zu einem Abfall der relativen Impedanzänderung; nach der Extubation ist ebenfalls ein deutlicher Abfall der relativen Impedanzänderung zu sehen.

Auch die Profilkurve (siehe Abb. 14) veranschaulicht wie die oben dargestellte Verlaufskurve den deutlichen Unterschied der relativen Impedanzänderung während der Beatmung und nach der Extubation. 


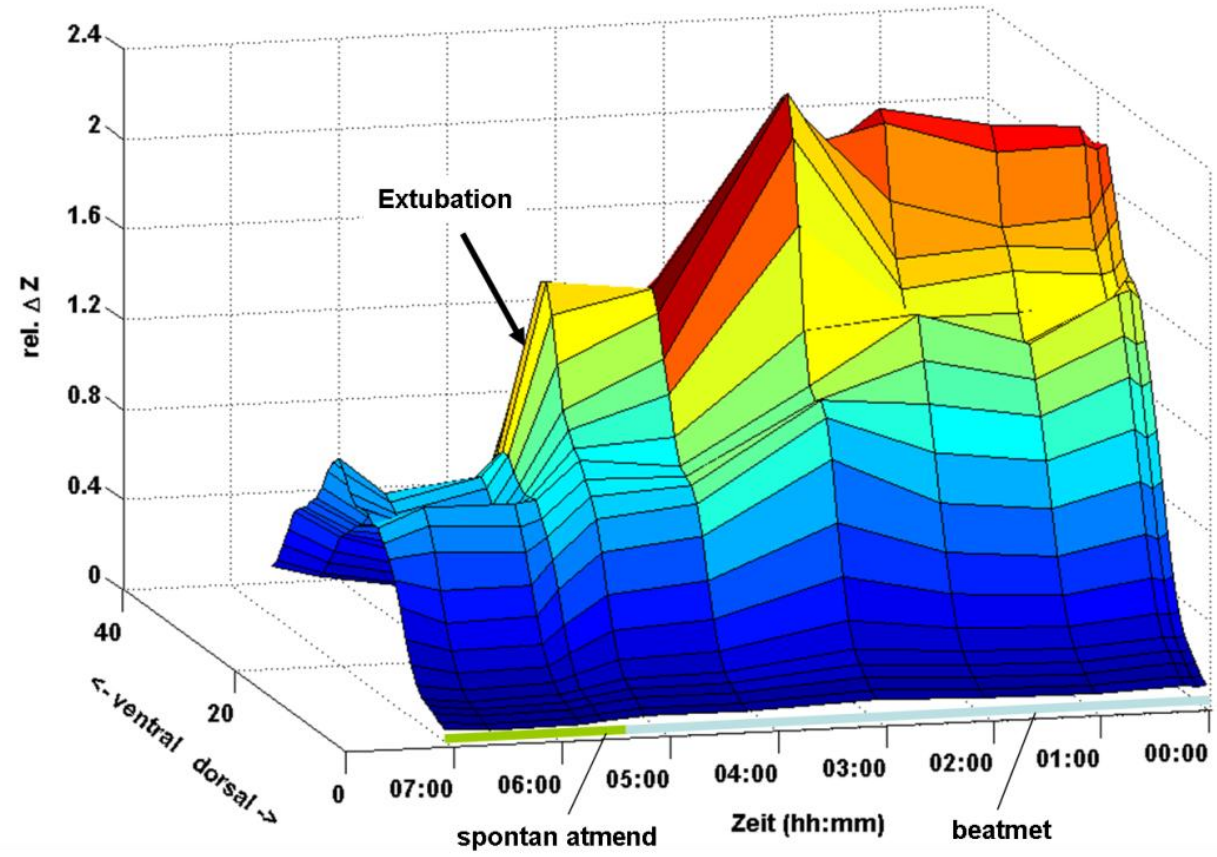

Abbildung 14: Verlauf der relativen Impedanzänderung (rel. $\Delta Z$ ) über den gesamten Messzeitraum (hh:mm) von Patient 13 für den ventralen und dorsalen Lungenbereich. Von rechts nach links verlaufende Zeitachse, um die geringeren relativen Impedanzänderungen im dorsalen Bereich sehen zu können. Unter Spontanatmung deutlich niedrigere relative Impedanzänderungen als unter Beatmung.

\subsection{Darstellung des rechten und linken Lungenflügels}

Die unterschiedlichen Ventilationsverhältnisse im rechten und linken Lungenflügel sowie deren zeitlichen Verlauf bei Patient 13 verdeutlichen die Verlaufs- und die Profilkurven (siehe Abb. 15 u. Abb. 16). Bis zur Extubation lagen bei dieser Patientin höhere relative Impedanzänderungen im linken Lungenflügel vor, während sich dieses Verhältnis nach der Extubation umkehrte und es zu einer Umverteilung kam: Nun waren im rechten Lungenflügel höhere relative Impedanzänderungen als im linken zu messen. 

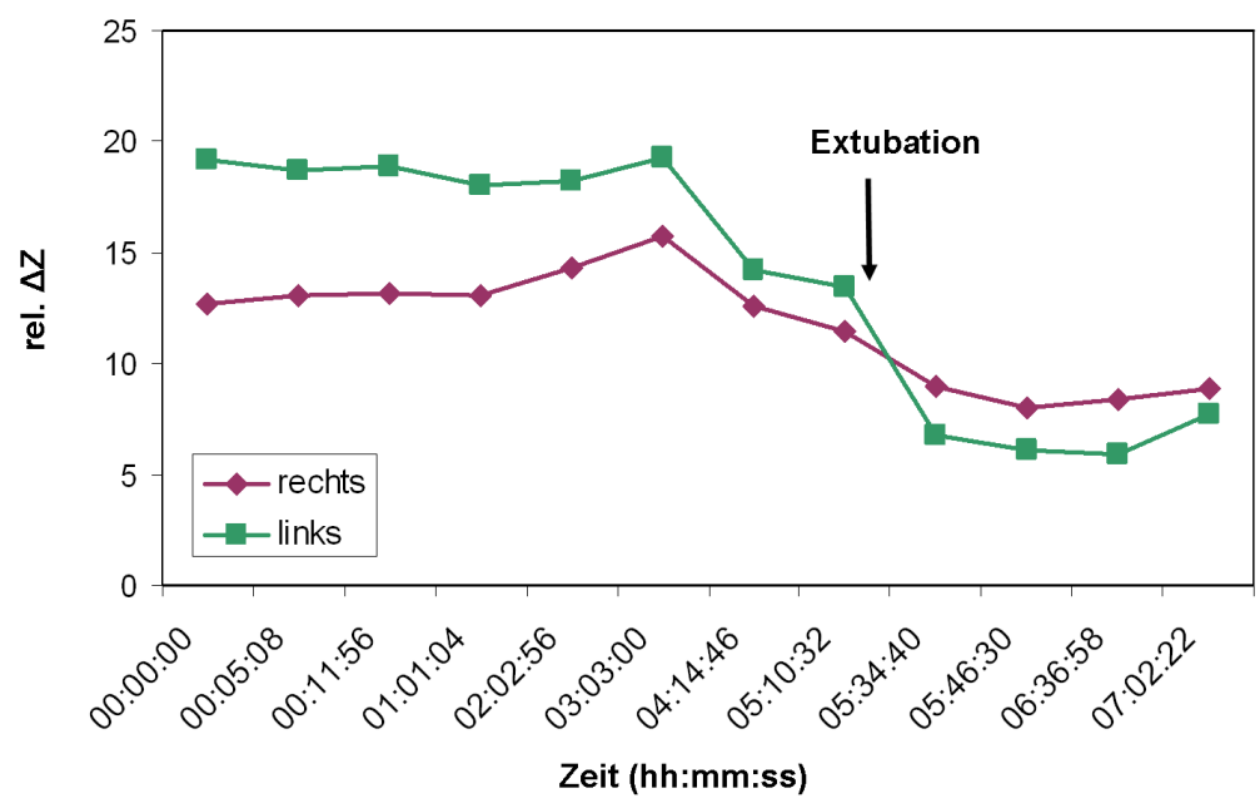

Abbildung 15: Verlauf der Summen relativen Impedanzänderung (rel. $\Delta Z$ ) des rechten und linken Lungenflügels über den Messzeitraum von Patient 13. Unter Beatmung höhere relative Impedanzänderung im linken Lungenflügel, nach der Extubation unter Spontanatmung Umkehrung dieses Verhältnisses.

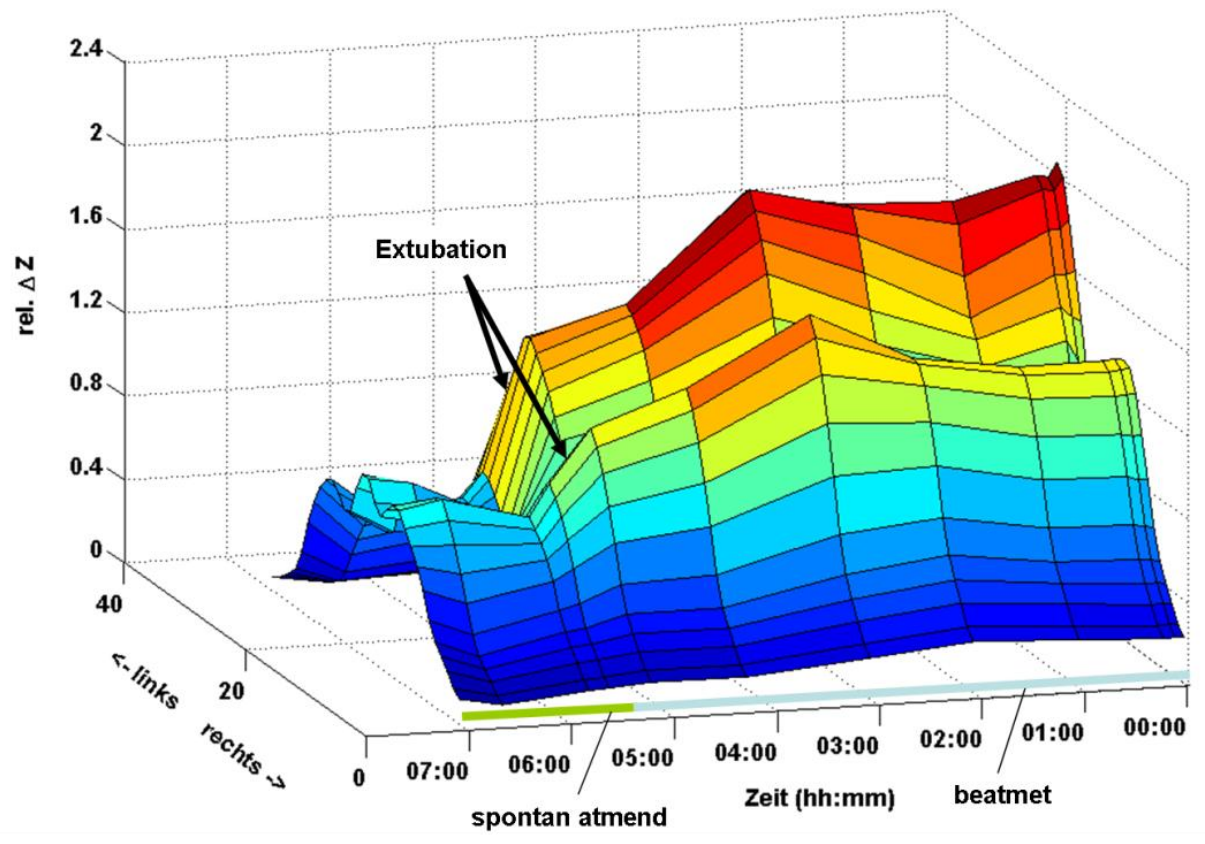

Abbildung 16: Verlauf der relativen Impedanzänderung (rel. $\Delta Z$ ) über den gesamten Messzeitraum (hh:mm) von Patient 13 für den rechten und linken Lungenflügel. Von rechts nach links verlaufende Zeitachse, um die geringeren relativen Impedanzänderungen im rechten Lungenflügel darstellen zu können. Unter Spontanatmung deutlich niedrigere relative Impedanzänderungen als unter Beatmung. 


\subsection{Darstellung der ventralen und dorsalen Lungenbereiche}

Auch die unterschiedlichen Ventilationsverhältnisse in den ventralen und dorsalen Lungenbereichen werden gut durch die Verlaufs- und Profilkurven dargestellt (siehe Abb. 14 u. Abb. 17). Sowohl unter Beatmung als auch während Spontanatmung wurden bei dieser Patientin in den ventralen Lungenbereichen höhere relative Impedanzänderungen gemessen als in den dorsalen Bereichen. Während der Beatmung waren diese Impedanz-Unterschiede zwischen ventraler und dorsaler Lunge deutlich größer als während der Spontanatmung.

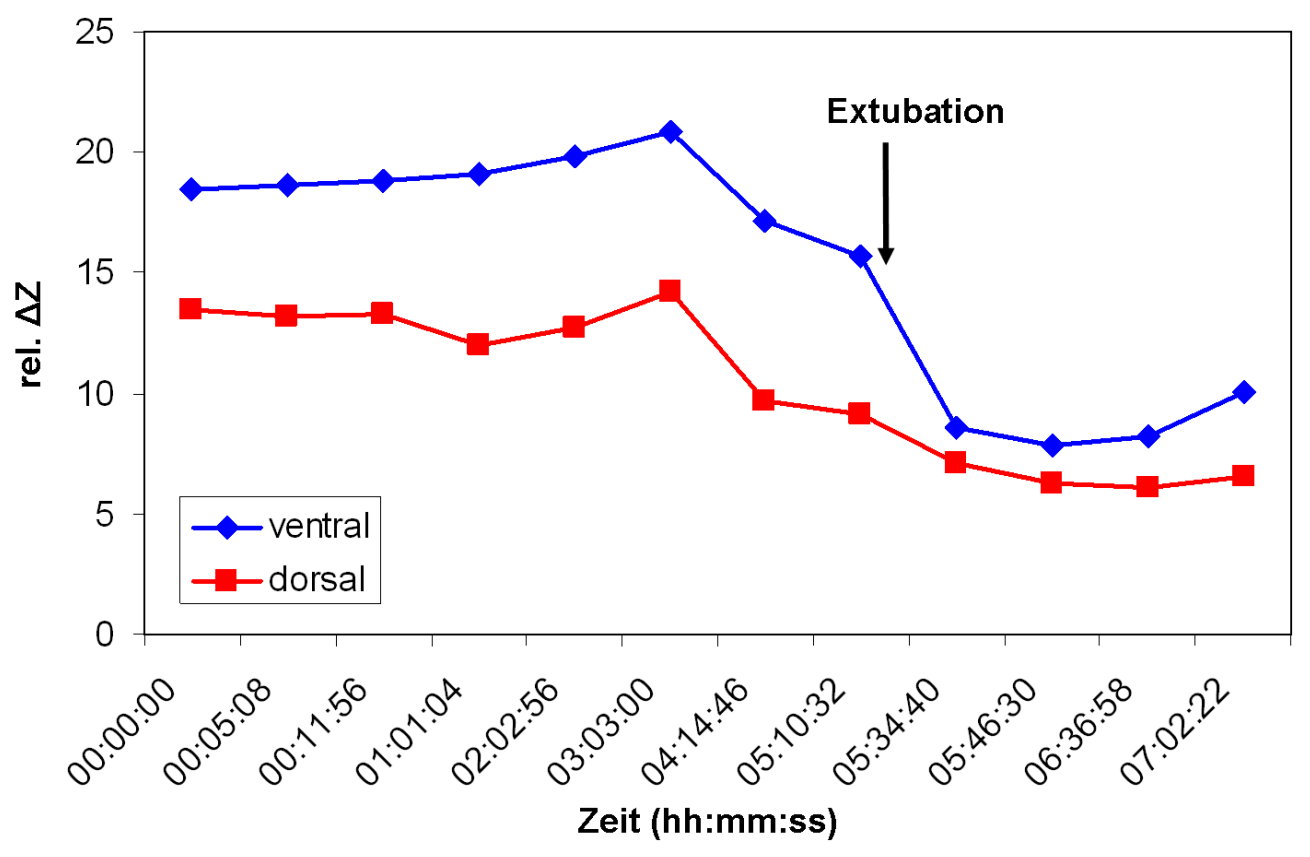

Abbildung 17: Verlauf der Summen der relativen Impedanzänderung (rel. $\Delta Z$ ) der ventralen und dorsalen Lungenabschnitte von Patient 13. Während Beatmung und bei Spontanatmung zeigen die ventralen Lungenabschnitte höhere relative Impedanzänderungen.

\subsection{Messungen über mehrere Tage}

7 der 29 Patienten mussten postoperativ länger als 24 Stunden maschinell beatmet werden. Die Profil- und Verlaufskurven weisen die Ventilationsschwankungen (z.B. durch Veränderungen der Respiratoreinstellungen oder unterschiedlich starke Eigenatmung des Patienten) über die verschiedenen Tage deutlich auf und sind 
genauso nachvollziehbar wie die Messungen über kürzere Zeiträume (siehe Abb. 18).

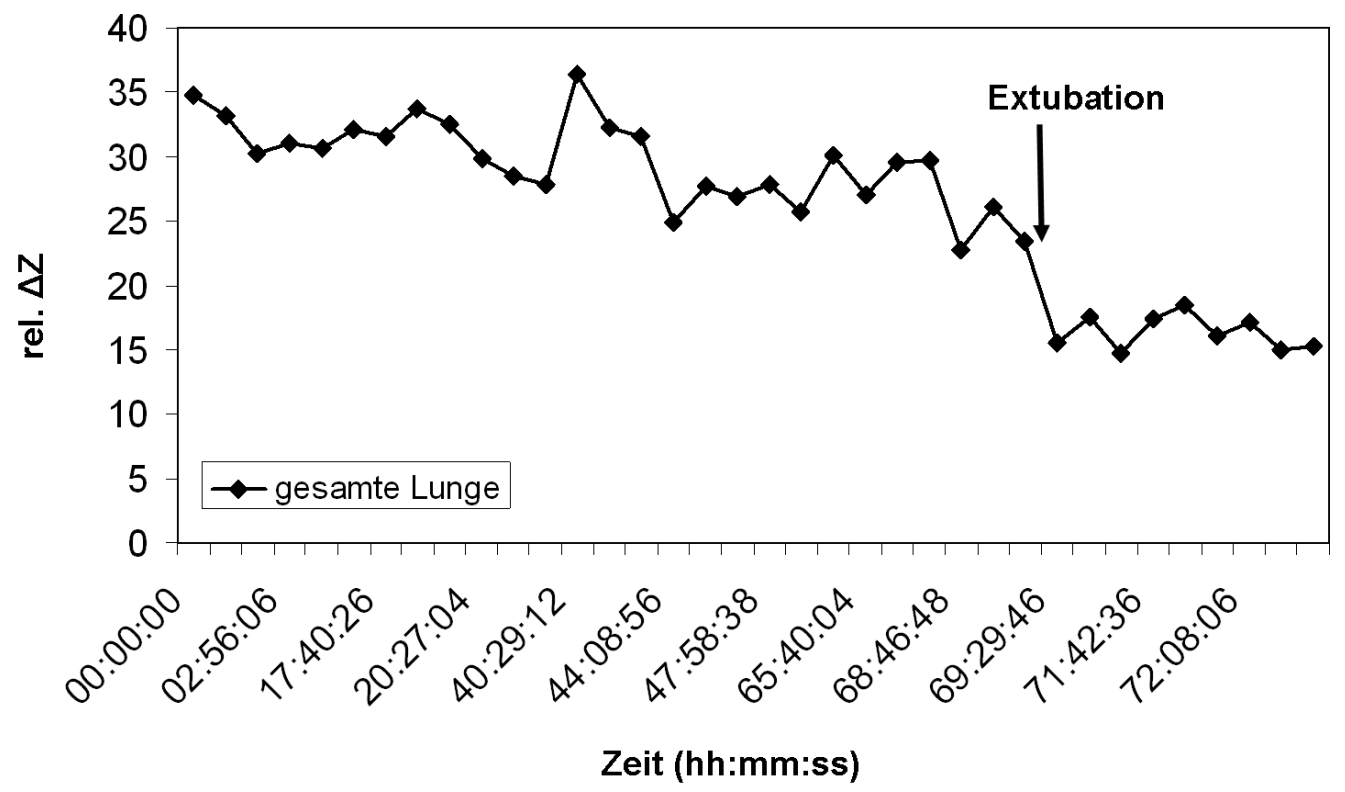

Abbildung 18: Verlauf der Summen der relativen Impedanzänderung (rel. $\Delta Z$ ) der gesamten Lunge über den Messzeitraum von Patient 6. Nach der Extubation ist ein deutlicher Abfall der relativen Impedanzänderung zu sehen.

\subsection{Visualisierung von Beatmungsmanövern und therapeutischen Eingriffen}

Wurde die maschinelle Beatmung von BIPAP- auf CPAP-Modus umgestellt oder der Tubus vom Beatmungssystem diskonnektiert, so sind diese „Manöver" in den Profilkurven deutlich zu erkennen. Beispielhaft ist dies im Folgenden für die Patienten 2 und 8 aufgeführt.

Bei Patient 2 (13-jähriger Junge, Aortenisthmusstenose, Z.n. Resektion der Aortenisthmusstenose) wurde die Beatmung kurz vor der Extubation von BIPAP - auf CPAP-Modus umgestellt. In dem Profilkurvenverlauf zeigt sich eine deutliche Abnahme der Ventilation für die drei Messungen der maschinellen Atemunterstützung im CPAP-Modus. Im CPAP-Modus war die Druckunterstützung um 3-6 mbar niedriger als im BIPAP-Modus (siehe Abb. 19). 


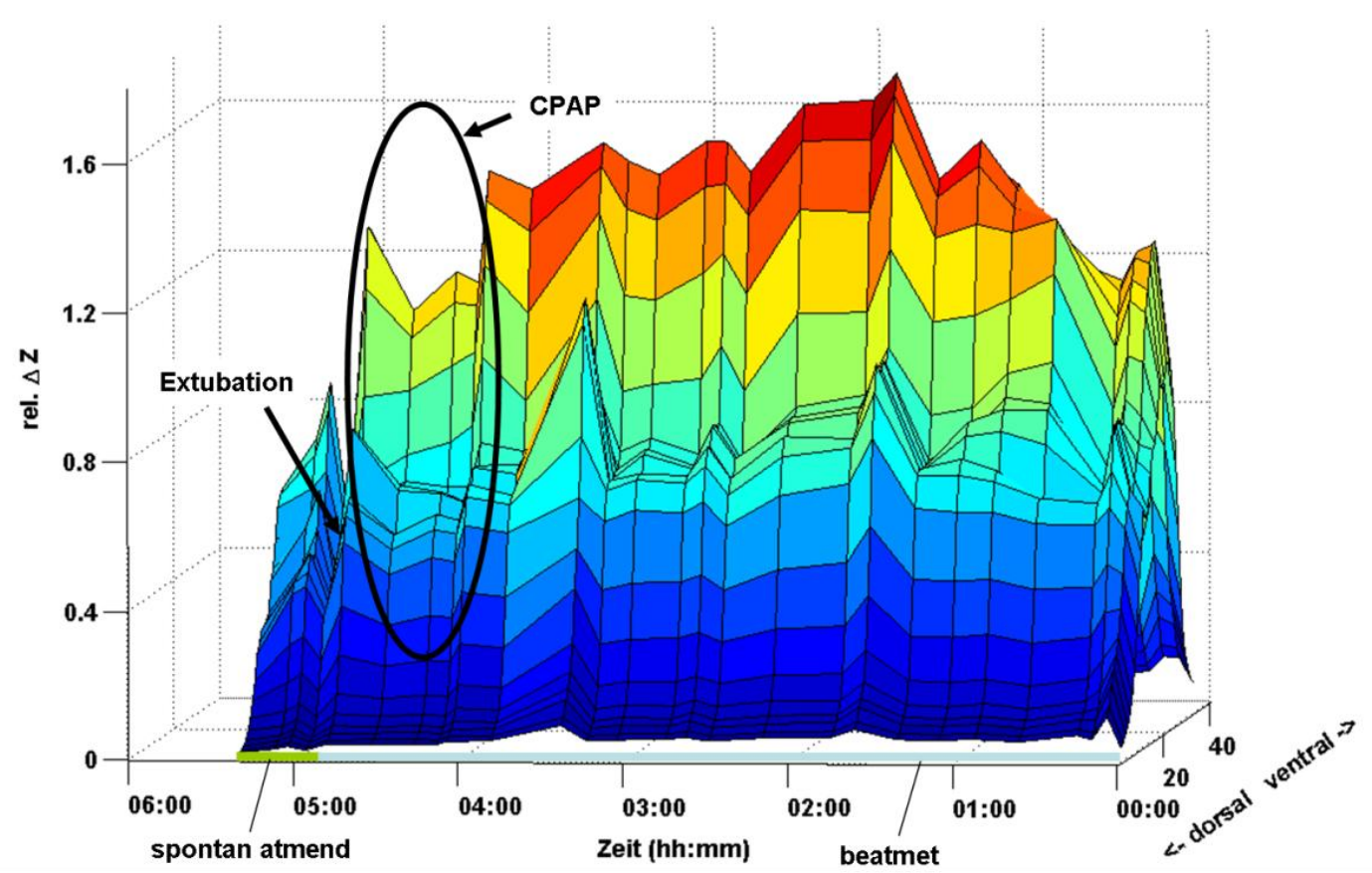

Abbildung 19: Verlauf der relativen Impedanzänderung (rel. $\Delta Z$ ) über den gesamten Messzeitraum (hh:mm) von Patient 2 für den ventralen und dorsalen Lungenbereich. Von rechts nach links verlaufende Zeitachse, um die geringeren relativen Impedanzänderungen in dem dorsalen Bereich sehen zu können. Nach Umstellung des Beatmungsmodus auf CPAP-Atmung (schwarze ovale Markierung) deutlich niedrigere relative Impedanzänderungen, unter anschließender Spontanatmung nochmals niedrigere relative Impedanzänderung.

Auch bei Patient 8 (7-jähriger Junge mit Shone-Komplex, Z.n. Resektion einer Subaortenstenose) wurde die Beatmung vor der Extubation von BIPAP - auf CPAPModus umgestellt, zusätzlich atmete der Patient während der letzten Messung vor der Extubation nach Trennung des Beatmungssystems vom Tubus spontan durch den Tubus. Beide Phänomene lassen sich anhand der Profilkurven nachvollziehen (siehe Abb. 20). Ein kurzfristiger Anstieg der Ventilation nach der Extubation lässt sich hier durch die klinische Beobachtung erklären, dass der Patient nach der Extubation agitiert war und zunächst hyperventilierte. 


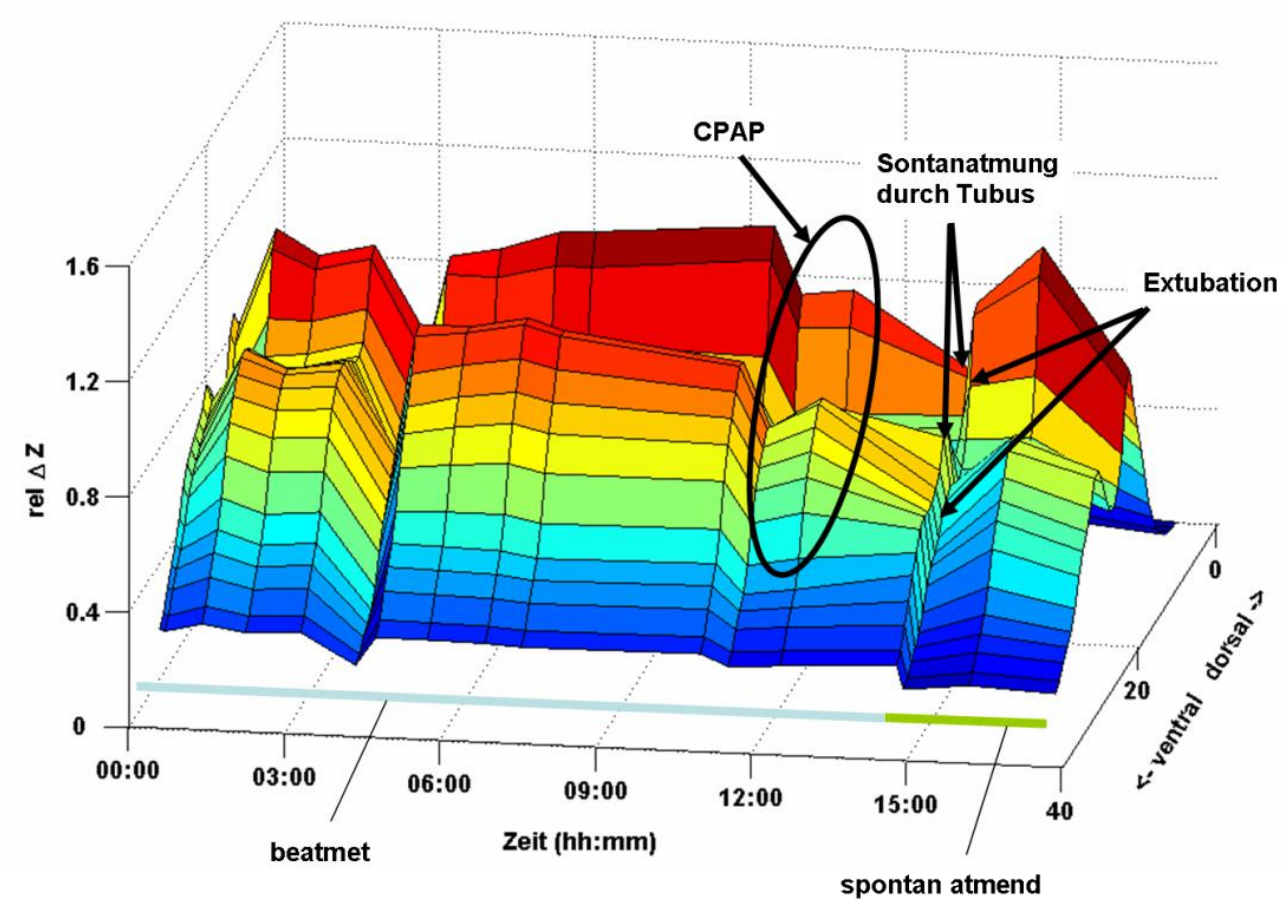

Abbildung 20: Verlauf der relativen Impedanzänderung (rel. $\Delta Z$ ) über den gesamten Messzeitraum (hh:mm) von Patient 8 für den ventralen und dorsalen Lungenbereich. Hier von links nach rechts verlaufende Zeitachse, um den ventralen Bereich besser darstellen zu können. Nach Umstellung des Beatmungsmodus auf CPAP-Atmung (schwarze ovale Markierung) deutlich niedrigere relative Impedanzänderungen, unter anschließender Spontanatmung über den Tubus (schwarze Pfeile) nochmals niedriger. Nach Extubation zunächst wieder höhere relative Impedanzänderungen.

Anhand der Profilkurven von Patient 15 (5-jähriger Junge mit Fallot'scher-Tetralogie und Pulmonalklappenagenesie, Z.n. Austausch eines Pulmonalis-Conduits) und Patient 9 (3-jähriger Junge mit ASD, Z.n. ASD-Patchverschluss) lässt sich der Effekt einer antiobstruktiven Inhalationsbehandlung dokumentieren. Bei beiden Patienten wurde nach der Extubation klinisch eine ausgeprägte bronchiale Obstruktion beobachtet, so dass sie mit bronchodilatatorisch wirksamen Substanzen (Salbutamol und Ipratropiumbromid) inhalativ (Feuchtvernebelung) behandelt wurden. In den Profilkurven (siehe Abb. 21 u. Abb. 22) ist während der Inhalationen eine Zunahme der relativen Impedanzänderung und somit der Ventilation zu sehen. Sobald die Inhalation beendet wurde, fielen die relative Impedanzänderung und folglich die Ventilation dort wieder $a b$. 


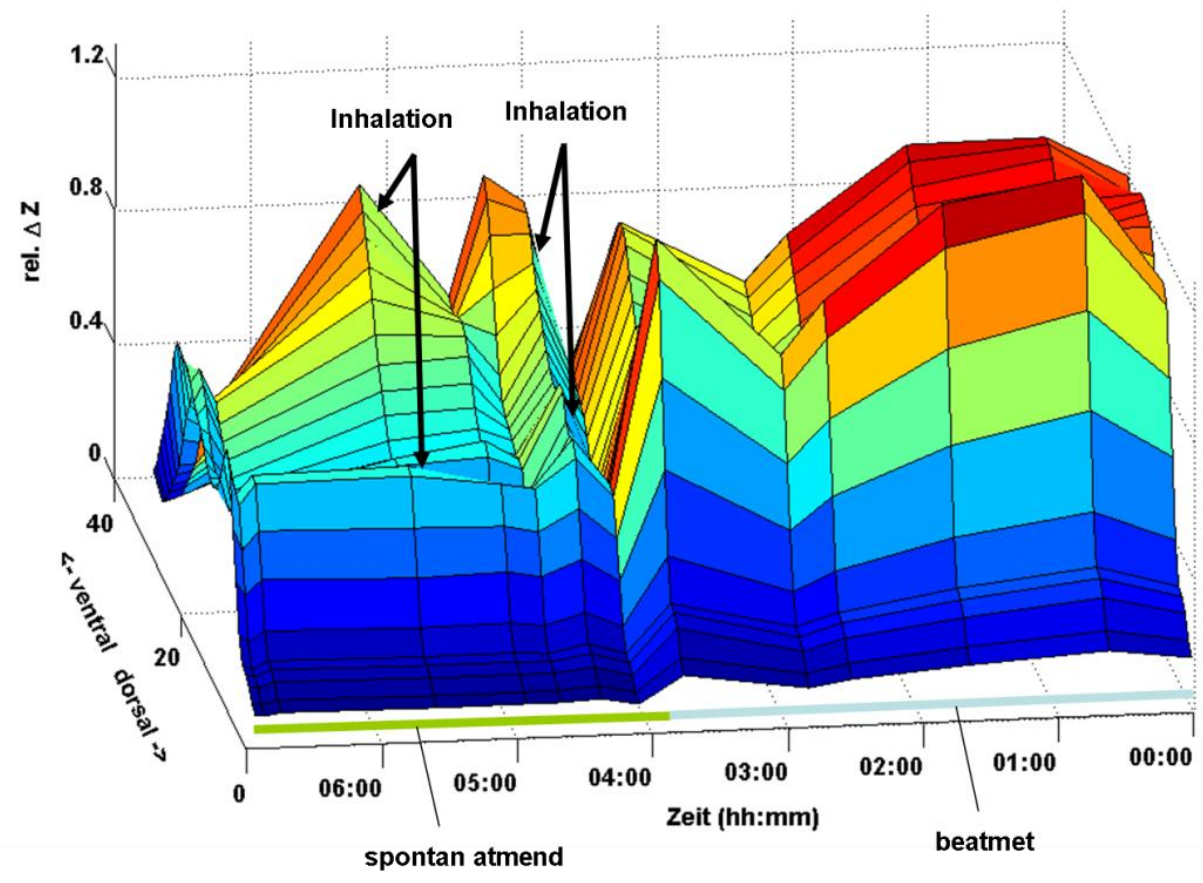

Abbildung 21: Verlauf der relativen Impedanzänderung (rel. $\Delta Z$ ) über den gesamten Messzeitraum (hh:mm) von Patient 15 für den ventralen und dorsalen Lungenbereich. Von rechts nach links verlaufende Zeitachse, um die unter Spontanatmung geringeren relativen Impedanzänderungen im dorsalen Bereich darstellen zu können. Nach Extubation Inhalation bei bronchialer Obstruktion (schwarze Pfeile), darunter deutliche Zunahme der relativen Impedanzänderungen im ventralen Lungenbereich. Nach den Inhalationen wieder Abfall. 


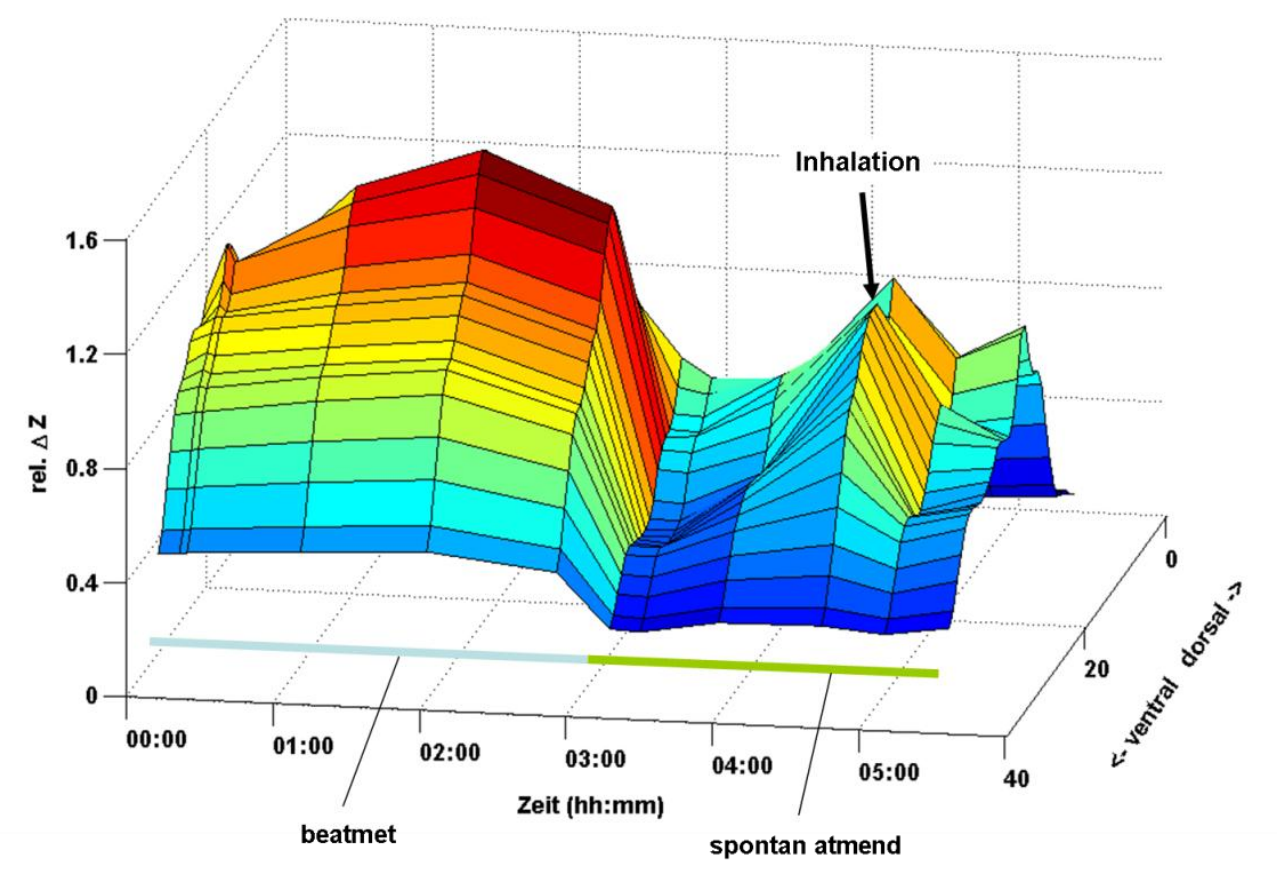

Abbildung 22: Verlauf der relativen Impedanzänderung (rel. $\Delta Z$ ) über den gesamten Messzeitraum (hh:mm) von Patient 9 für den ventralen und dorsalen Lungenbereich. Von links nach rechts verlaufende Zeitachse. Nach Extubation Inhalation bei bronchialer Obstruktion (schwarze Pfeile), darunter deutliche Zunahme der relativen Impedanzänderungen im ventralen und dorsalen Lungenbereich. Nach den Inhalationen wieder Abfall.

\subsection{Darstellung der Ergebnisse für das Patientenkollektiv}

Der Umfang des ausgewerteten Gesamtdatensatzes (29 Patienten) für die Messung der relativen Impedanzänderung und parallele Erfassung von Beatmungsparametern betrug $n=654$ Messungen (davon $n=444$ im beatmeten Zustand, $n=210$ im spontan atmenden Zustand). Dieser Datensatz wurde einer mehrfaktoriellen Varianzanalyse unterworfen (GLM), um erstens den erwarteten starken Effekt des Atmungsmodus (maschinelle Beatmung, Spontanatmung) zu überprüfen und zweitens mögliche Einflüsse der Patienten auf die Varianz festzustellen.

Insgesamt konnten 91,4\% der Datensätze genutzt werden, der Rest musste aufgrund von starken Artefakten (Bewegung der Patienten, Elektrodenartefakte) verworfen werden. Von den ausgewerteten Datensätzen (91,4\% des Gesamtdatensatzes) wurden insgesamt 24,3\% aufgrund von oben genannten Artefakten bearbeitet, indem die Sequenzen mit Artefakten entfernt wurden; somit konnten auch diese Datensätze 
der Auswertung zugänglich gemacht werden. Auffällig war, dass der Anteil der genutzten Datensätze bei den 0-3-jährigen Kindern geringer war als bei den älteren Kindern und dass vor allem bei den Säuglingen wesentlich mehr Datensätze bearbeitet werden mussten (siehe Tab. 3).

Tabelle 3: Anteil genutzter und bearbeiteter Datensätze in Abhängigkeit vom Alter der Patienten. Mittelwerte und p-Werte (Kruskal-Wallis Test).

\begin{tabular}{|c|c|c|c|}
\hline Altersgruppe & $\begin{array}{c}\text { Anteil } \\
\text { genutzter } \\
\text { Datensätze } \\
(\%)\end{array}$ & $\begin{array}{c}\text { Anteil } \\
\text { bearbeiteter } \\
\text { Datensätze } \\
(\%)\end{array}$ & Anzahl Patienten \\
\hline 0 Jahre & 87,7 & 39,0 & 7 \\
\hline 1-3 Jahre & 87,3 & 27,7 & 6 \\
\hline 4-18 Jahre & 94,5 & 16,6 & 16 \\
\hline Gesamt & 91,4 & 24,3 & 29 \\
\hline p & 0,030 & 0,015 & \\
\hline
\end{tabular}

\subsubsection{Bedeutung von maschineller Beatmung, Spontanatmung und individuellen Patientencharakteristika für die relative Impedanzänderung}

Im Mittel ergab sich eine relative Impedanzänderung von 20,8 \pm 6,2 während der maschinellen Beatmung und 14,1 \pm 4,1 nach der Extubation unter Spontanatmung $(p<0,001)$. Der partielle Eta ${ }^{2}$-Wert zeigte, dass der Atmungsmodus (Beatmung/ Spontanatmung) mit 70,2\% hohe Anteile der Varianz erklärt. Aber auch individuelle Patientencharakteristika wie z.B. das Alter trugen erheblich zur Varianz der Werte bei $\left(p=0,004 ; \mathrm{Eta}^{2}=73,6 \%\right)$. Außerdem ergab sich eine statistisch signifikante Interaktion zwischen Atmungsmodus und Patient $\left(\mathrm{p}<0,001\right.$; $\left.\mathrm{Eta}^{2}=34,1 \%\right)$, die darauf hinweist, dass auch das Ausmaß der Veränderung in der relativen Impedanzänderung vom beatmeten zum spontan atmenden Zustand durch den Patienten beeinflusst wurde.

Daraus folgt, dass Einflüsse der individuellen Patientencharakteristika auf die relative Impedanzänderung bei den weiteren statistischen Analysen berücksichtigt werden mussten. Deshalb wurden die Ergebnisse je Patient gemittelt und Vergleiche innerhalb eines Patienten als verbundene Stichproben mit t-Tests für verbundene Stichproben bzw. Varianzanalysen für Messwertwiederholung (GLMRM) geprüft.

Auf die Beatmungsparameter wird in Abschnitt 3.6.8 auf Basis des Gesamtdatensatzes eingegangen. 


\subsubsection{Relative Impedanzänderung während Beatmung und Spontanatmung}

Die Mittelwerte der relativen Impedanzänderung der Patienten unter Beatmung und unter Spontanatmung zeigten, dass die relative Impedanzänderung mit Ausnahme von Patient 23 bei jedem Patienten nach der Extubation unter Spontanatmung insgesamt abnahm (siehe Tab. 4 u. Abb. 23). Das Ausmaß der Abnahme ist dabei für jeden einzelnen Patienten unterschiedlich.

Tabelle 4: Mittelwerte der relativen Impedanzänderung (rel. $\Delta Z) \pm$ Standardabweichung für jeden Patienten im beatmeten und spontan atmenden Zustand. In Klammern steht die Anzahl der Messungen (siehe auch Abb. 23 u. Abb. 24).

\begin{tabular}{|c|c|c|}
\hline Patient & $\begin{array}{c}\text { rel. } \Delta \mathrm{Z} \\
\text { beatmet } \pm \text { SD }\end{array}$ & $\begin{array}{c}\text { rel. } \Delta Z \\
\text { spontan atmend } \pm S D\end{array}$ \\
\hline 1 & $20 \pm 2(16)$ & $14 \pm 3(3)$ \\
\hline 2 & $21 \pm 3(23)$ & $11 \pm 2(4)$ \\
\hline 3 & $26 \pm 3(22)$ & $19 \pm 1(8)$ \\
\hline 4 & $35 \pm 3(10)$ & $18 \pm 1(4)$ \\
\hline 5 & $17 \pm 1(6)$ & $14 \pm 2(6)$ \\
\hline 6 & $30 \pm 3(27)$ & $16 \pm 1(9)$ \\
\hline 7 & $21 \pm 3(27)$ & $16 \pm 1(13)$ \\
\hline 8 & $17 \pm 3(14)$ & $13 \pm 2(3)$ \\
\hline 9 & $23 \pm 2(6)$ & $14 \pm 3(6)$ \\
\hline 10 & $16 \pm 1(14)$ & $13 \pm 2(9)$ \\
\hline 11 & $22 \pm 4(12)$ & $16 \pm 1(3)$ \\
\hline 12 & $18 \pm 3(10)$ & $12 \pm 3(7)$ \\
\hline 13 & $31 \pm 3(8)$ & $15 \pm 1(4)$ \\
\hline 14 & $13 \pm 2(13)$ & $9 \pm 1(5)$ \\
\hline 15 & $23 \pm 4(6)$ & $15 \pm 3(8)$ \\
\hline 16 & $16 \pm 2(57)$ & $12 \pm 1(8)$ \\
\hline 17 & $15 \pm 2(14)$ & $13 \pm 3(15)$ \\
\hline 18 & $25 \pm 1(6)$ & $16 \pm 1(7)$ \\
\hline 19 & $25 \pm 2(5)$ & $19 \pm 2(6)$ \\
\hline 20 & $22 \pm 2(18)$ & $15 \pm 2(4)$ \\
\hline 21 & $32 \pm 9(8)$ & $15 \pm 2(5)$ \\
\hline 22 & $17 \pm 3(29)$ & $16 \pm 7(9)$ \\
\hline 23 & $12 \pm 2(17)$ & $14 \pm 6(11)$ \\
\hline 24 & $19 \pm 0(5)$ & $10 \pm 1(5)$ \\
\hline 25 & $28 \pm 1(15)$ & $13 \pm 2(14)$ \\
\hline 26 & $23 \pm 3(29)$ & $19 \pm 9(7)$ \\
\hline 28 & $13 \pm 3(12)$ & $7 \pm 1(10)$ \\
\hline 29 & $19 \pm 7(9)$ & $13 \pm 2(8)$ \\
\hline 30 & $27 \pm 2(10)$ & $13 \pm 1(7)$ \\
\hline
\end{tabular}




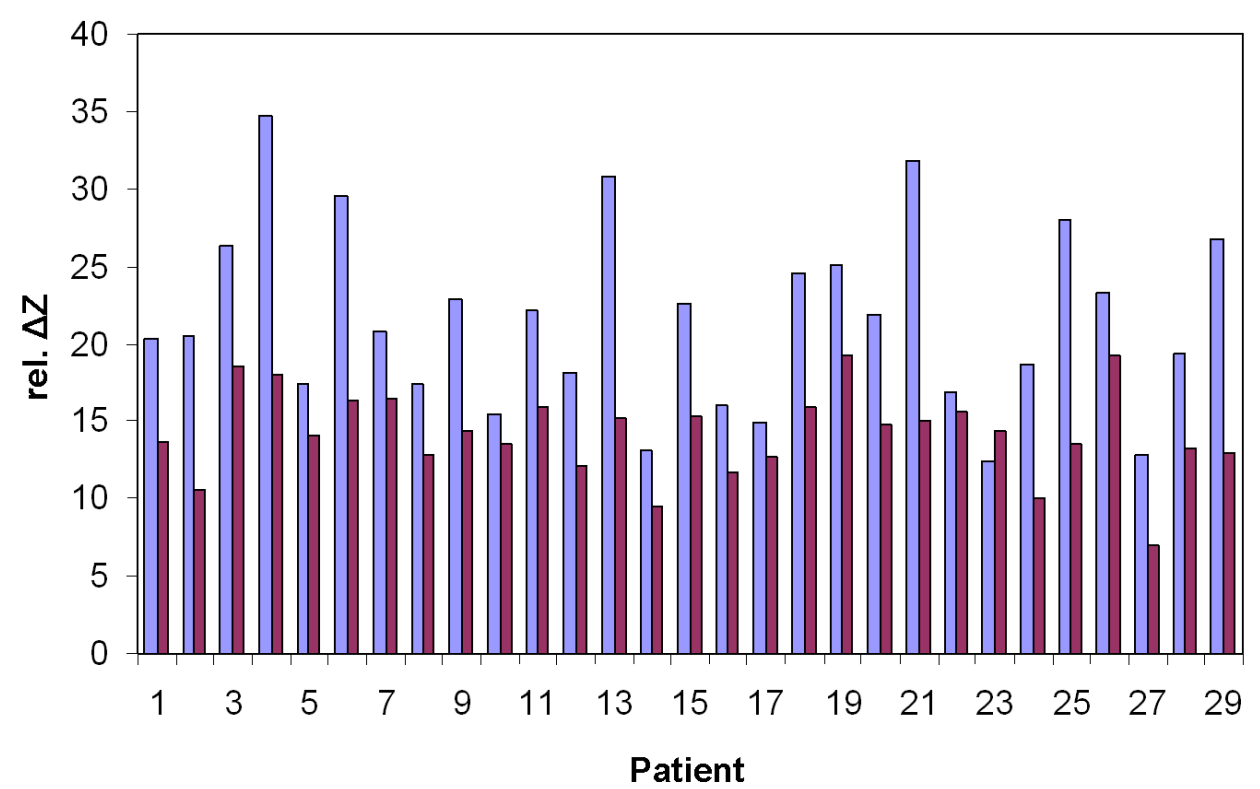

Abbildung 23: Mittelwerte der relativen Impedanzänderung (rel. $\Delta \mathrm{Z}$ ) für jeden einzelnen der $n=29$ Patienten im beatmeten (blauer Balken) und spontan atmenden Zustand (violetter Balken). Bei allen Patienten, mit Ausnahme von Patient 23, Abnahme der relativen Impedanzänderung unter Spontanatmung.

\subsubsection{Mittelwerte und prozentuale Änderung}

Die Mittelwerte der relativen Impedanzänderung waren unter maschineller Beatmung mit 21,6 \pm 6,0 signifikant höher als unter Spontanatmung nach Extubation mit 14,1 \pm 2,9 (siehe Abb. 24), die Ventilation nahm somit nach der Extubation deutlich ab (tTest, $\mathrm{p}<0,001)$. 


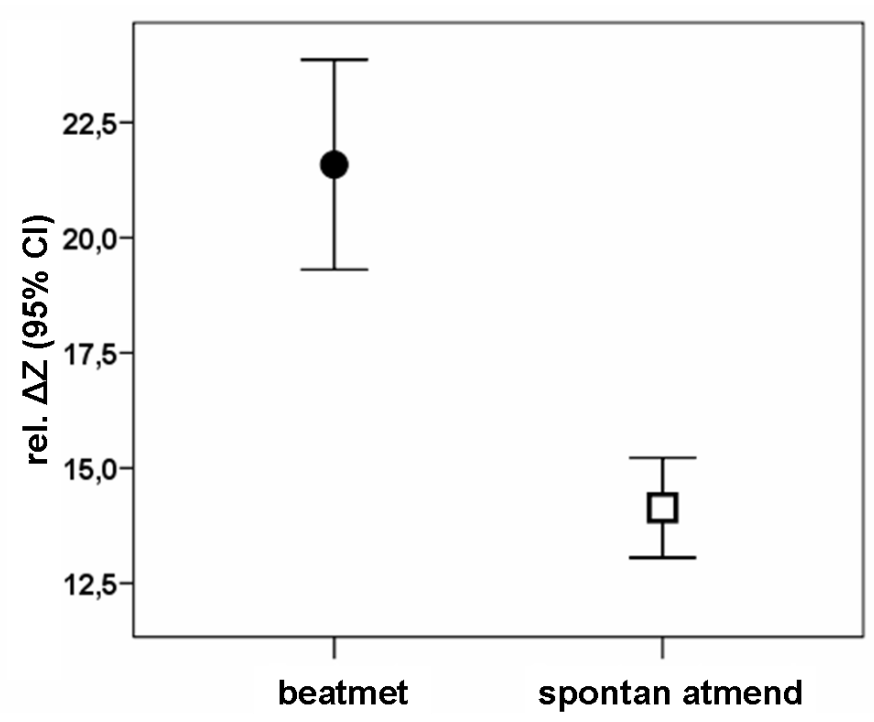

Abbildung 24: Mittelwerte der relativen Impedanzänderung (rel. $\Delta Z$ ) für insgesamt $n=29$ Patienten jeweils im beatmeten $(\bullet)$ und spontan atmenden $(\square)$ Zustand. Unter Spontanatmung zeigt sich eine signifikante Abnahme der Mittelwerte der relativen Impedanzänderung.

Die prozentuale Abnahme der Mittelwerte der relativen Impedanzänderung nach Extubation betrug im Mittel für alle untersuchten Patienten $32 \pm 16 \%$ (Minimum 17\%, Maximum 54\%).

Die prozentuale Änderung war bei den älteren Kindern relativ groß (Kleinkinder $38 \pm$ $16 \%$, Schulkinder/Jugendliche $36 \pm 10 \%$ ) und bei den Säuglingen mit $16 \pm 19 \%$ am geringsten und damit signifikant unterschiedlich zu den älteren Kindern (ANOVA, $p=0,011$; Scheffé-Test, Altersgruppen 1-3 Jahre: $p=0,041 ; 4-18$ Jahre: $p=0,017 ; 1-3$ Jahre gegen 4-18 Jahre: $p=0,978$, siehe dazu auch 3.6.2.3).

\subsubsection{Variation der Werte der relativen Impedanzänderung der Patienten unter Beatmung und unter Spontanatmung}

Die Variation der Messwerte betrachtet für jeden einzelnen Patienten, angegeben durch den patientenspezifischen Variationskoeffizienten (vgl. Tab. 5), war zwischen Beatmung und Spontanatmung nicht signifikant verschieden und hing auch nicht vom Alter ab (GLMRM, $p=0,089$; Interaktion zwischen Beatmungszustand und Alter: $p=0,435$; Altersgruppe als Zwischensubjekteffekt: $p=0,231$ ). Es deutete sich aber eine höhere Variation der Werte unter Spontanatmung insbesondere bei den 
Säuglingen und Kleinkindern an. Diese wäre sicherlich noch größer gewesen, wenn die Artefakte nicht aus den gestörten Datensätzen entfernt worden wären (siehe Patienten und Methoden, 2.6).

Tabelle 5: Variationskoeffizienten der relativen Impedanzänderung bei Patienten verschiedenen Alters im beatmeten und spontan atmenden Zustand.

\begin{tabular}{|c|c|c|}
\hline Altersgruppe & Beatmung & Spontanatmung \\
\hline 0 Jahre $(n=7)$ & $0,15 \pm 0,05$ & $0,22 \pm 0,16$ \\
\hline 1-3 Jahre $(n=6)$ & $0,13 \pm 0,08$ & $0,18 \pm 0,15$ \\
\hline 4-18 Jahre $(n=16)$ & $0,12 \pm 0,09$ & $0,13 \pm 0,06$ \\
\hline Gesamt $(n=29)$ & $0,13 \pm 0,08$ & $0,16 \pm 0,11$ \\
\hline
\end{tabular}

Ob die Variation der relativen Impedanzänderung von der Messdauer abhängig war, wird im Folgenden beschrieben: Bei 7 der 29 Patienten war eine Beatmung länger als 24 Stunden notwendig, daher erfolgte auch die Datenaquisition über den gesamten Zeitraum der Beatmung. Da Schulkinder/Jugendliche ausschließlich kürzer als 24 Stunden lang untersucht wurden, wurden hier nur die jüngeren Kinder in Abhängigkeit von der Messdauer verglichen. Dabei zeigten sich keine signifikanten Unterschiede der Variation der relativen Impedanzänderung in Abhängigkeit von der Messdauer (beatmet < 1d: 0,15 $\pm 0,09, n=6 ;>1 d: 0,12 \pm 0,04, n=7, p=0,438$, ANOVA; spontan atmend < 1d: 0,19 $\pm 0,13, n=6$; > 1d: 0,21 $\pm 0,18, n=7, p=0,762)$.

Tabelle 6: Variationskoeffizient der Impedanzänderung in Abhängigkeit von der Messdauer bei den jüngeren Kindern (0-1-Jährige, 1-3-Jährige).

\begin{tabular}{|c|c|c|}
\hline Messdauer & Beatmung & Spontanatmung \\
\hline$<1 d(n=6)$ & $0,16 \pm 0,09$ & $0,19 \pm 0,13$ \\
\hline$>1 d(n=7)$ & $0,12 \pm 0,04$ & $0,21 \pm 0,18$ \\
\hline insgesamt $(n=13)$ & $0,14 \pm 0,06$ & $0,20 \pm 0,15$ \\
\hline
\end{tabular}

\subsubsection{Unterschiede der relativen Impedanzänderung unter Beatmung und Spontanatmung}

Zwischen den Patienten-Mittelwerten der relativen Impedanzänderung unter Beatmung und unter Spontanatmung bestand ein signifikanter positiver 
Zusammenhang $\left(r_{p}=0,579, p=0,001\right)$ : Hatte ein Patient während der maschinellen Beatmung höhere relative Impedanzänderungen, so lagen diese auch unter Spontanatmung höher (siehe Abb. 25). Dieser Zusammenhang trat in jeder Altersgruppe auf, war aber besonders deutlich bei den 4-18 Jahre alten Kindern zu erkennen. Die Werte unter Beatmung lagen bei den älteren Patienten (1-18 Jahre) wie oben ausgeführt (siehe 3.6.2.1) wesentlich höher und umfassten einen größeren Bereich der relativen Impedanzänderung als unter Spontanatmung (vgl. Abb. 25 u. Tab. 4). Spontan atmende Patienten wiesen unabhängig von der Altersgruppe niedrigere relative Impedanzwerte auf (siehe auch 3.6.6).

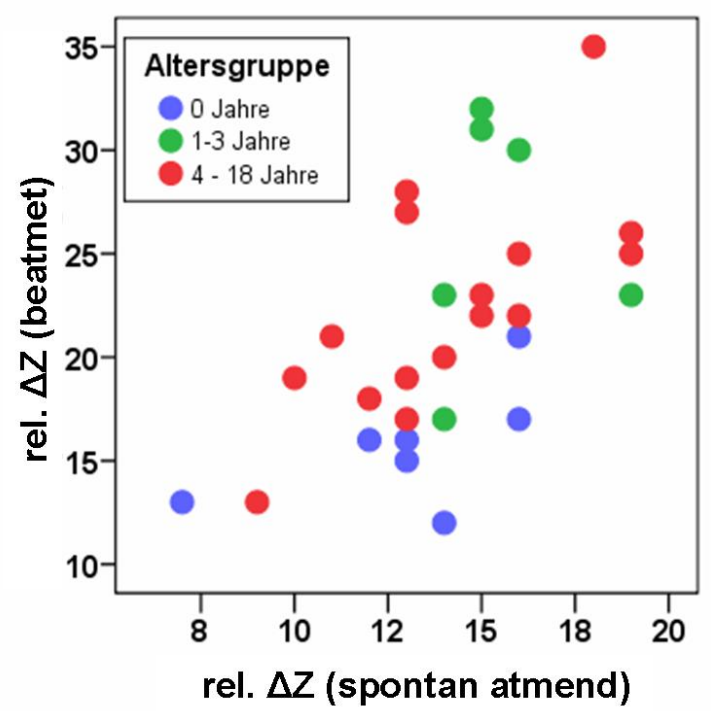

Abbildung 25: Zusammenhang der Mittelwerte der relativen Impedanzänderung (rel. $\Delta Z$ ) unter Beatmung und unter Spontanatmung, farblich dargestellt sind die drei Altersgruppen. Unter Beatmung hohe relative Impedanzänderungen gehen mit ebenfalls höheren Werten unter Spontanatmung einher. Man beachte die unterschiedlichen Skalen.

\subsubsection{Geschlechtsunterschiede}

Um die Frage einer möglichen Geschlechterabhängigkeit der gemessenen Impedanzänderungen zu beantworten, wurden die Ergebnisse weiblicher und männlicher Patienten verglichen. Die Mittelwerte der relativen Impedanzänderung unter Beatmung der weiblichen Patienten von 22,3 \pm 6,2 $(n=9)$ und der männlichen von $21,3 \pm 6,0 \quad(n=20)$ zeigten keinen signifikanten Unterschied (t-Test für 
unverbundene Stichproben, $p=0,661)$. Auch die Mittelwerte unter Spontanatmung der weiblichen Patienten von 13,8 $\pm 2,3$ und der männlichen Patienten von 14,3 $\pm 3,1$ zeigten keinen signifikanten Geschlechtsunterschied $(p=0,656)$.

\subsubsection{Seitenvergleich rechter Lungenflügel - linker Lungenflügel}

Die Mittelwerte der relativen Impedanzänderung des rechten Lungenflügels betrugen im beatmeten Zustand 10,6 \pm 2,7 und nach Extubation unter Spontanatmung 7,4 \pm 1,8, während die Mittelwerte für den linken Lungenflügel im beatmeten Zustand 11,0 $\pm 3,4$ und unter Spontanatmung 7,0 $\pm 1,9$ betrugen. Somit gab es weder im Zeitraum der maschinellen Beatmung ( $\mathrm{t}$-Test, $\mathrm{p}=0,286$ ) noch im Zeitraum der Spontanatmung $(p=0,190)$ einen signifikanten Unterschied in der Ventilation des rechten und des linken Lungenflügels.

\subsubsection{Unterschiede zwischen ventralen und dorsalen Lungenbereichen}

Insgesamt war der ventrale Lungenbereich sowohl im beatmeten als auch im spontan atmenden Zustand signifikant besser ventiliert als die dorsale Lunge, wie die Mittelwerte der relativen Impedanzänderungen anzeigten (siehe Abb. 26; t-Test, beatmet: $p<0,001$; spontan atmend: $p=0,001$ ). 


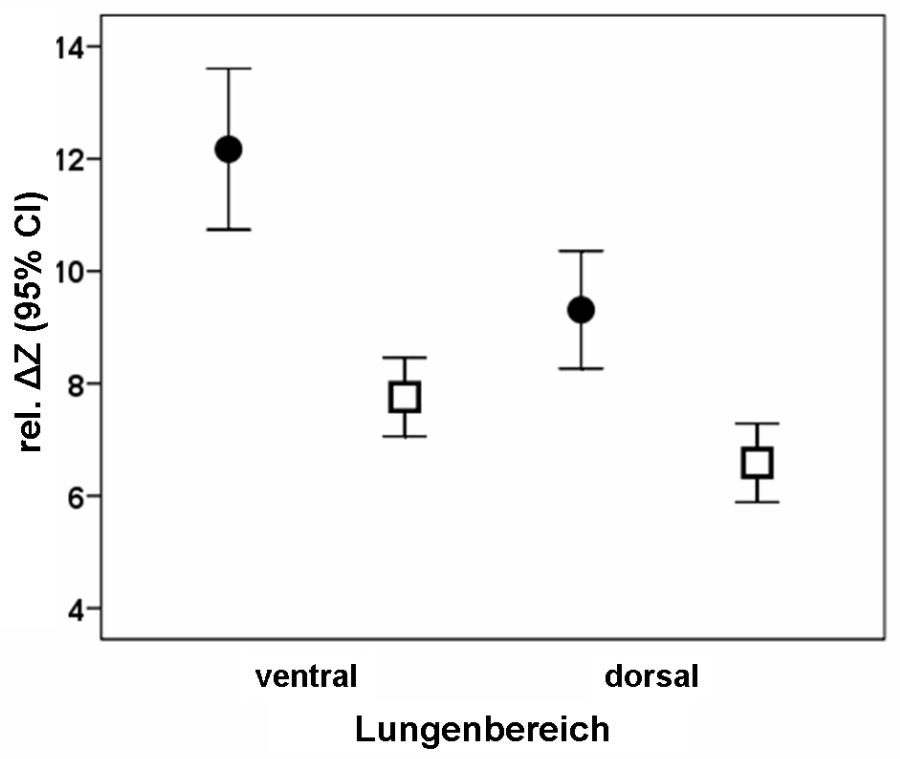

Abbildung 26: Mittelwerte der relativen Impedanzänderung (rel. $\Delta Z$ ) jeweils im ventralen und dorsalen Lungenbereich erst unter Beatmung $(\bullet)$ und dann unter Spontanatmung ( $\square$ ) für insgesamt $n=29$ Patienten.

Außerdem zeigten die Daten (siehe Abb. 26), dass im ventralen Lungenbereich der absolute Impedanzunterschied zwischen beatmeten Patienten und spontan atmenden Patienten signifikant größer war als in dorsalen Lungenbereichen (ventral $4,4 \pm 3,2$; dorsal $2,7 \pm 2,1$, t-Test, $p<0,001$ ). Dies galt auch für den relativen Unterschied, bezogen auf die während maschineller Beatmung gemessenen Werte: Die Änderung in den ventralen Lungenbereichen war um 6\% signifikant höher als in den dorsalen Abschnitten (ventral 0,33 $\pm 0,17$, dorsal $0,27 \pm 0,17$; $t$-Test, $p=0,017$ ).

Der prozentuale Unterschied zwischen dorsaler und ventraler Ventilation hing nicht vom Alter der Patienten ab, sondern war in jedem Alter in gleicher Größenordnung vorhanden (GLMRM, $p=0,801$ ).

\subsubsection{Abhängigkeit der relativen Impedanzänderung vom Alter}

Der unter Abschnitt 3.6.2 beschriebene Unterschied der relativen Impedanzänderung zwischen Beatmung und Spontanatmung war signifikant vom Alter der Patienten abhängig ( $p=0,005$, GLMRM). Die Säuglinge wiesen einen nur geringen Abfall der Mittelwerte von 15,7 $\pm 2,7$ unter Beatmung auf 13,0 $\pm 3,1$ unter Spontanatmung auf, während die Mittelwerte bei den Kleinkindern von 26,0 \pm 6,0 auf 15,5 \pm 1,9 fielen und bei den Schulkindern/ Jugendlichen von $22,5 \pm 5,2$ auf 14,1 $\pm 3,0$ (siehe Abb. 27). 
Dabei fiel auf, dass die Unterschiede der relativen Impedanzänderung zwischen beatmeten und spontan atmenden Patienten in den beiden älteren Altersgruppen deutlich größer waren als bei den jüngsten Patienten (ANOVA, $p=0,002$; Altersgruppe der Säuglinge signifikant verschieden von den beiden älteren Altersklassen). Bemerkenswerterweise war die relative Impedanzänderung in der Gruppe der 1-3-jährigen Patienten nicht niedriger, sondern eher höher (aber nicht signifikant) als in der Gruppe der 4 -18-jährigen Patienten. In dieser Gruppe wurden 7 der insgesamt 16 Patienten einer Re-Operation unterzogen. Die re-operierten Patienten wiesen eine mit 18,8 deutlich niedrigere relative Impedanzänderung auf als die übrigen 9 Kinder mit erstmaliger Thoraxoperation mit 25,3. Die niedrigere relative Impedanzänderung der re-operierten Patienten hat somit den Mittelwert in dieser Altersgruppe insgesamt verringert. Des Weiteren fiel besonders auf, dass sich die Mittelwerte der relativen Impedanzänderung während Spontanatmung zwischen den einzelnen Altersklassen nicht signifikant unterschieden $(p=0,299)$.

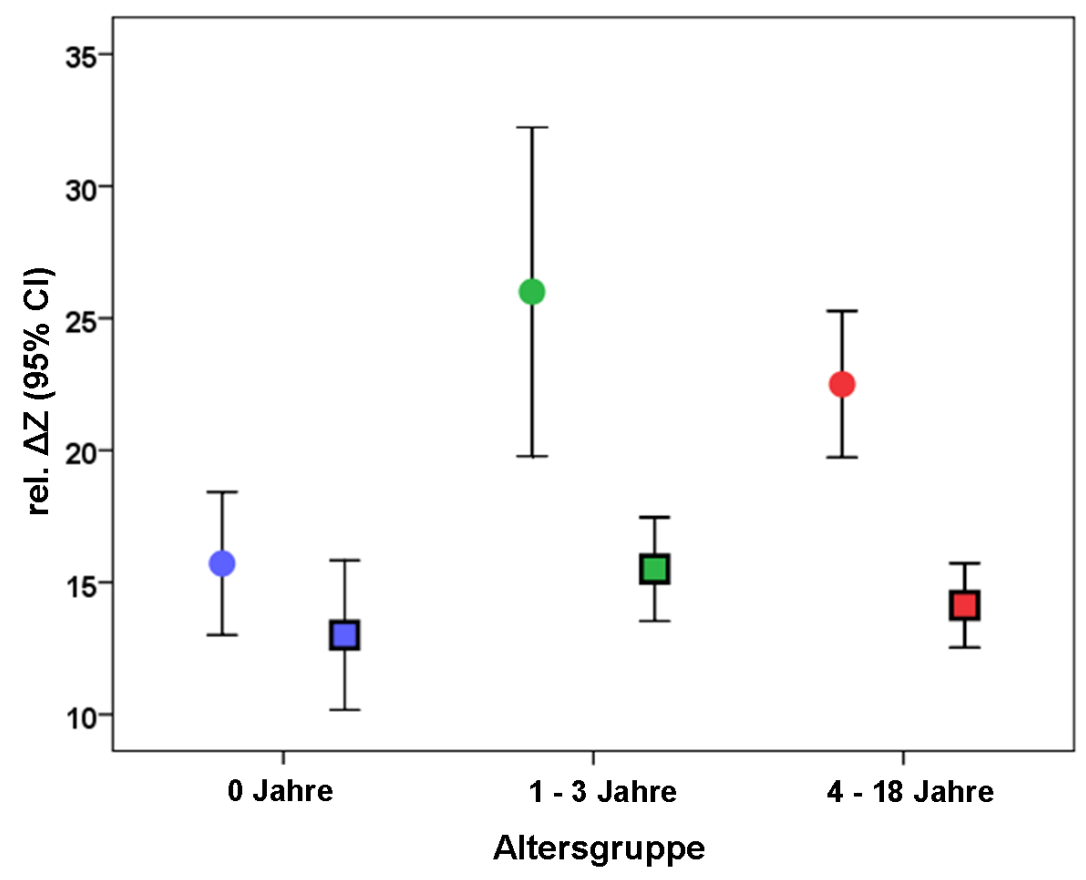

Abbildung 27: Mittelwerte der relativen Impedanzänderung (rel. $\Delta Z$ ) für insgesamt $n=29$ Patienten aufgeteilt in die drei Altersgruppen 0 Jahre, 1-3 Jahre und 4-18 Jahre im beatmeten (Kreis) und spontan atmenden Zustand (Kasten).

Im Gegensatz zum spontan atmenden Zustand korrelierte bei den beatmeten Patienten die relative Impedanzänderung positiv mit dem Alter (siehe Tab. 7). 
Tabelle 7: Korrelationen der mittleren relativen Impedanzänderung (rel. $\Delta Z$ ) im beatmeten und spontan atmenden Zustand untereinander und mit dem Alter der Patienten (n=29). Korrelationskoeffizienten nach Pearson. Signifikante Ergebnisse sind durch Fettdruck hervorgehoben.

\begin{tabular}{|l|c|c|}
\hline & $\begin{array}{c}\text { Mittlere rel. } \Delta Z \\
\text { beatmet }\end{array}$ & $\begin{array}{c}\text { Mittlere rel. } \Delta Z \\
\text { spontan atmend }\end{array}$ \\
\hline $\begin{array}{l}\text { Mittlere rel. } \Delta Z \\
\text { spontan atmend }\end{array}$ & $\mathbf{0 , 5 7 9}(\mathrm{p}=0,001)$ & -- \\
\hline Alter & $\mathbf{0 , 3 9 2 ( p = 0 , 0 3 6 )}$ & $0,066(\mathrm{p}=0,733)$ \\
\hline
\end{tabular}

\subsubsection{Korrelation des $\mathrm{CO}_{2}$-Partialdruckes mit der relativen Impedanzänderung}

Bei 23 von den 29 Patienten konnten während des gesamten Messzeitraumes arterielle Blutgasanalysen durchgeführt werden (siehe Patienten und Methoden, 2.5). Es sollte untersucht werden, ob Änderungen der relativen Thoraximpedanz und damit Ventilationsänderungen mit Veränderungen des arteriellen $\mathrm{CO}_{2}$-Partialdruckes $\left(\mathrm{p}_{\mathrm{a}} \mathrm{CO}_{2}\right.$, Blutgasparameter für die Ventilation) korrelierten.

Zunächst wurde untersucht, ob sich ein signifikanter Unterschied des $\mathrm{p}_{\mathrm{a}} \mathrm{CO}_{2}$ unter Spontanatmung verglichen mit dem Zeitraum der maschinellen Beatmung ergibt. Die Mittelwerte des $\mathrm{p}_{2} \mathrm{CO}_{2}$ waren bei den 23 Patienten unter Beatmung mit 39,3 $\pm 3,2$ $\mathrm{mmHg}$ signifikant geringer als unter Spontanatmung mit $42,7 \pm 4,3 \mathrm{mmHg}$ (t-Test, $\mathrm{p}=0,003$ ). Unabhängig davon, ob die Patienten maschinell beatmet waren oder spontan atmeten, zeigte sich eine negative Korrelation zwischen der relativen Impedanzänderung und den $\mathrm{CO}_{2}$-Partialdrücken im Blut: Hohe Impedanzänderungen bei beatmeten Patienten korrelierten mit niedrigen $\mathrm{p}_{\mathrm{a}} \mathrm{CO}_{2}$-Werten, während in spontan atmendem Zustand hohe $\mathrm{p}_{\mathrm{a}} \mathrm{CO}_{2}$-Werte und geringe relative Impedanzänderungen gemessen wurden (siehe Abb. 28). Insgesamt ergab sich ein Korrelationskoeffizient von $r_{s}=-0,314$. Eine statistische Analyse war aufgrund des Vorliegens abhängiger Werte nicht zulässig.

Es ergab sich weder ein signifikanter Zusammenhang für die beatmeten $\left(r_{s}=-0,235\right.$, $p=0,280)$ noch für die spontan atmenden Patienten $\left(r_{s}=-0,005, p=0,982\right)$. 


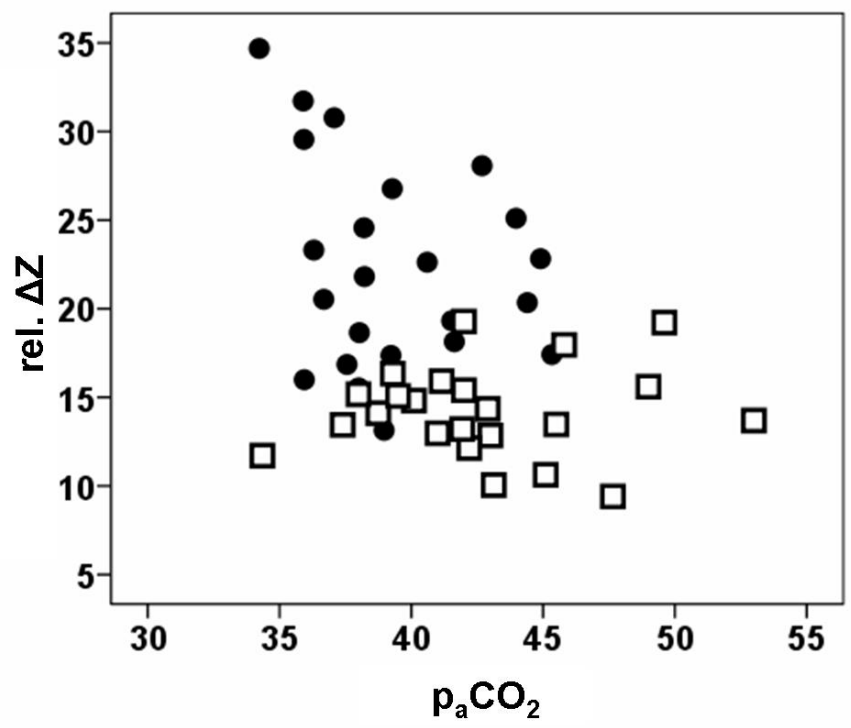

Abbildung 28: Mittelwerte der relativen Impedanzänderung (rel. $\Delta Z$ ) in Zusammenhang mit den Mittelwerten des $p_{a} \mathrm{CO}_{2}$ für jeden der $n=23$ Patienten jeweils unter Beatmung $(\bullet)$ und unter Spontanatmung $(\square)$. Es zeigt sich ein negativer Zusammenhang: Je höher der $\mathrm{p}_{a} \mathrm{CO}_{2}$, desto niedriger die relative Impedanzänderung.

Für den $\mathrm{p}_{\mathrm{a}} \mathrm{O}_{2}$ und die transkutane Sauerstoffsättigung $\left(\mathrm{S}_{\mathrm{a}} \mathrm{O}_{2}\right)$ wurden keine Korrelationen mit der relativen Impedanzänderung getestet, da diese von der inspiratorischen Sauerstoffkonzentration $\left(\mathrm{FiO}_{2}\right)$ beeinflusst wurden, die bei jedem Patienten zu unterschiedlichen Messzeitpunkten je nach Sauerstoffbedarf unterschiedlich war und somit keinen Vergleich im Gesamtkollektiv möglich machte.

\subsubsection{Zusammenhänge zwischen der relativen Impedanzänderung und den Beatmungsparametern}

Für die gemittelte Atemfrequenz zeigte sich ein signifikanter negativer Zusammenhang mit der mittleren relativen Impedanzänderung (siehe Tab. 8 u. Abb. 29): Je höher die Atemfrequenz, desto geringer war die relative Impedanzänderung.

Für die gemittelte Druckamplitude (Differenz zwischen Inspirationsdruck und PEEP) ergab sich ein tendenziell positiver Zusammenhang mit der mittleren relativen Impedanzänderung, der jedoch nicht signifikant war (siehe Tab. 8 u. Abb. 30). 
Tabelle 8: Zusammenhänge zwischen der mittleren relativen Impedanzänderung und der mittleren Atemfrequenz und Druckamplitude unter Beatmung. Signifikante Korrelationen im Fettdruck.

\begin{tabular}{|l|l|c|c|c|}
\hline \multicolumn{1}{|c|}{ Parameter } & Zustand & $\begin{array}{l}\text { Korrelations- } \\
\text { koeffizient } \mathbf{r}_{\mathbf{s}}\end{array}$ & $\mathbf{p}$ & $\mathbf{n}$ \\
\hline Atemfrequenz & beatmet & $\mathbf{- 0 , 5 4 6}$ & $\mathbf{0 , 0 0 2}$ & 29 \\
\hline Druckamplitude ( $\mathbf{P}_{\text {insp }}$-PEEP) & beatmet & 0,271 & 0,156 & 29 \\
\hline
\end{tabular}

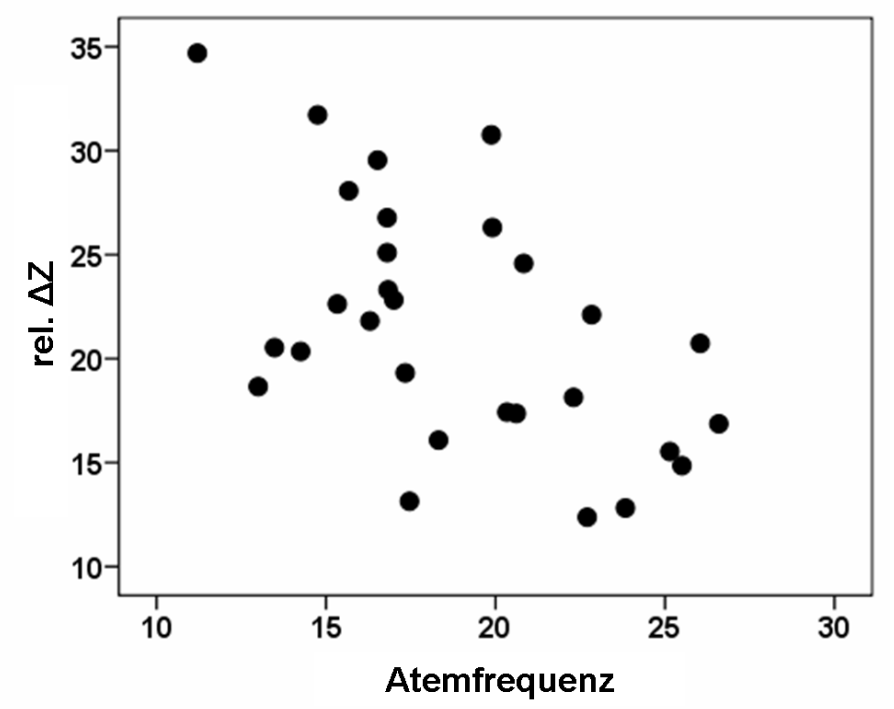

Abbildung 29: Zusammenhang der Mittelwerte der relativen Impedanzänderung (rel. $\triangle Z$ ) mit den Mittelwerten der Atemfrequenz in dem Zeitraum der maschinellen Beatmung für $n=29$ Patienten. Hohe Änderungen der relativen Impedanz gehen mit niedrigen Atemfrequenzen einher, während niedrige relative Impedanzänderungen mit hohen Atemfrequenzen einhergehen. 


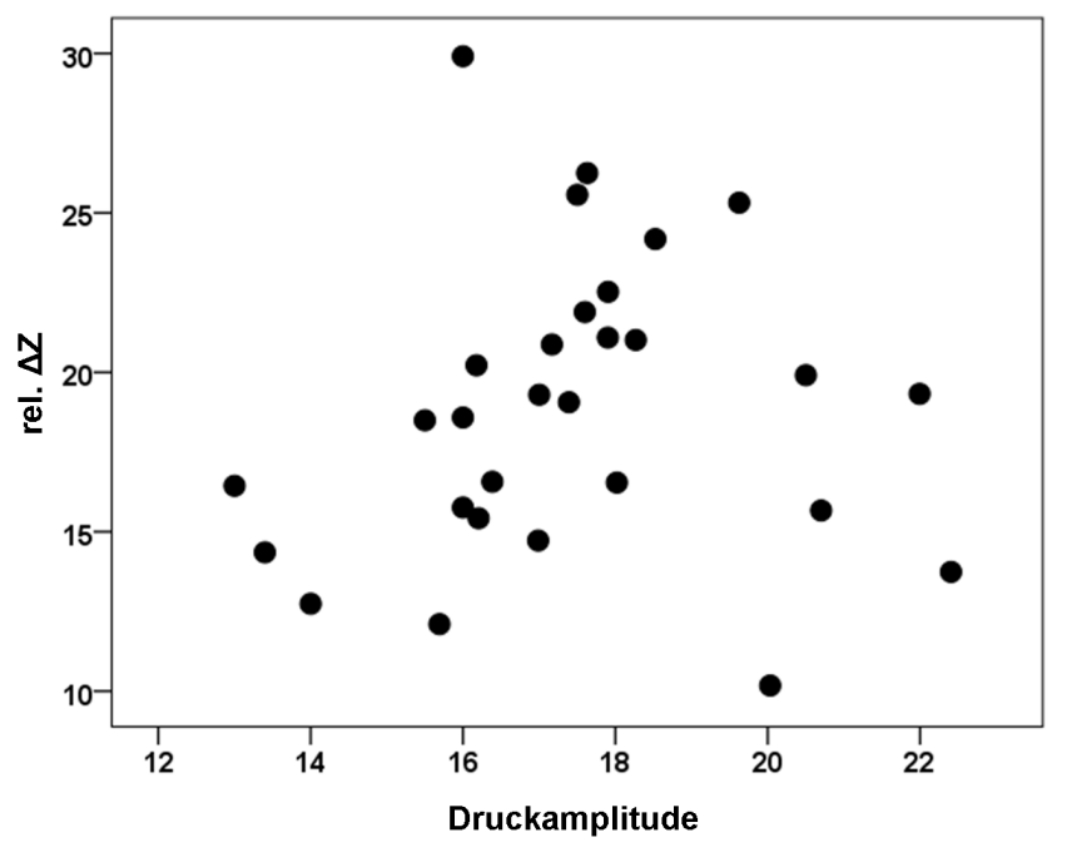

Abbildung 30: Zusammenhang der Mittelwerte der relativen Impedanzänderung (rel. $\triangle Z$ ) mit den Mitte/werten der Druckamplitude in dem Zeitraum der maschinellen Beatmung für $n=29$ Patienten.

Die Korrelationen zwischen den Beatmungsparametern und der relativen Impedanzänderung betrachtet für den gesamten Datensatz zeigten einen ähnlichen Zusammenhang wie bei den Patientenmittelwerten, erwiesen sich aber als schwach (s. Tab. 9; nicht auf Signifikanz getestet aufgrund der Mehrfachmessungen je Patient). Im Falle der Atemfrequenz war der Korrelationskoeffizient im Vergleich zu den Werten auf Patientenbasis (vgl. Tab. 9) erniedrigt.

Tabelle 9: Zusammenhänge zwischen der relativen Impedanzänderung und Beatmungsparametern von 29 Patienten in Zusammenhang mit Beatmung und Spontanatmung.

\begin{tabular}{|l|c|c|c|}
\hline \multicolumn{1}{|c|}{ Parameter } & Zustand & $\begin{array}{c}\text { Korrelations- } \\
\text { koeffizient } \mathbf{r}_{\mathbf{s}}\end{array}$ & $\mathbf{n}$ \\
\hline Atemfrequenz & beatmet & $-0,284$ & 444 \\
\hline Druckamplitude $\left(\mathbf{P}_{\text {insp }}\right.$-PEEP) & beatmet & 0,275 & 444 \\
\hline
\end{tabular}




\section{Diskussion}

Die vorliegende Arbeit befasste sich mit der Frage, ob mit Hilfe der elektrischen Impedanztomographie $(\mathrm{ET})$ eine Überwachung der Beatmung bei pädiatrischen Patienten nach einem Herz-Thoraxeingriff möglich ist. Dieses wurde an einem im Vergleich zu anderen pädiatrischen EIT-Studien [Dunlop et al. 2006, Frerichs et al. 1999b, 2001, 2003a, Hampshire et al. 1995, Heinrich et al. 2006, Marven et al. 1996, Schibler et al. 2009, Taktak et al. 1996] umfangreicheren Patientenkollektiv von 29 herz- und gefäßoperierten, intubierten und maschinell beatmeten Kindern bis über den Zeitpunkt der Extubation hinaus untersucht. Lediglich Brown et al. (2002) haben an einem großen Kollektiv von 142 Säuglingen EIT-Studien durchgeführt; Riedel et al. (2009) und Pham et al. (2011) haben mit 32 Neugeborenen sowie Humphreys et al. (2011) mit 38 Säuglingen und Kindern ein vergleichbar großes Kollektiv wie in der vorliegenden Arbeit untersucht. Wie in der Studie von Humphreys et al. (2011) wurden in dieser Arbeit nicht nur Neugeborene und Säuglinge, sondern auch Kinder und Jugendliche aller Altersstufen mit der EIT untersucht. Des Weiteren existiert bisher keine EIT-Studie an Kindern nach einem großen thoraxchirurgischen Eing riff.

Im Folgenden sollen nun diese Punkte erörtert werten:

1) Ist die Methode der EIT unter den gegebenen Rahmenbedingungen anwendbar?

2) Sind die Resultate vereinbar mit den Erwartungen; inwiefern zeigt sich eine Übereinstimmung der Ergebnisse mit den publizierten Daten?

3) Ist die Anwendbarkeit der EIT speziell bei herz- und gefäßoperierten Kindern und Jugendlichen gegeben und welche Vorschläge ergeben sich daraus für die weitere Entwicklung der Methode?

\subsection{Bewertung und Auswertung der Daten dieser Arbeit}

Insgesamt konnte ein repräsentativer Anteil von 91,4\% der erhobenen Daten für die Auswertung genutzt werden. Die Aquisition aussagekräftiger Messwerte bei Neugeborenen und Säuglingen stellte sich als aufwendiger dar als bei den größeren Kindern, da die Patienten dieser Altersgruppe insbesondere in der Phase vor und nach der Extubation wesentlich unruhiger waren. Dieses führte häufiger zu Bewegungsartefakten und daraus resultierenden Elektrodenfehlern in den 
gewonnenen Messdaten, weshalb die Anzahl der nicht verwertbaren Datensätze bei den Neugeborene und Säuglingen deutlich höher war als bei den älteren Kindern und Jugendlichen. Um auch die Daten nutzen zu können, die nur teilweise durch Artefakte gestört waren, wurden die Artefakte aus den Datensätzen entfernt (siehe Patienten und Methoden, 2.6). Der Anteil der Datensätze, welche Artefakte enthielten war, wie erwartet, ebenfalls bei den Säuglingen am höchsten. Dieser Befund deutete sich zum Beispiel durch tendenziell erhöhte Variationskoeffizienten bei den Säuglingen unter Spontanatmung an und wäre sicher deutlicher gewesen, wenn diese Daten nicht aussortiert und die Sequenzen mit Artefakten entfernt worden wären (siehe Patienten und Methoden, 2.6 und Ergebnisse, 3.6).

Diese Studie profitierte von der schnellen Entwicklung und angestrebten Vereinfachung in den Auswertungs- und Bearbeitungsmöglichkeiten der EIT. Die graphische Darstellung der EIT-Daten ist mittels der durch die EIT-Group Göttingen erstmalig entwickelten Software zur Erstellung von Profilkurven und Profilverläufen sowie der in Excel erstellbaren Verlaufskurven sehr gut möglich. Hierdurch sind die Verläufe der relativen Impedanzänderungen während des Beobachtungszeitraumes gut beurteilbar. Die Möglichkeit, den Verlauf der relativen Impedanzänderungen anhand der Profilkurven dreidimensional und beliebig drehbar für verschiedene Lungenbereiche (dorsal/ ventral und rechter/ linker Lungenflügel) darstellen zu können, ist bislang nicht veröffentlicht worden und stelt einen wesentlichen Fortschritt in der Präsentation und Beurteilung der EIT-Daten dar.

Die in Göttingen für die Aufbereitung der Daten entwickelten und in dieser Arbeit genutzten Programme zum Bearbeiten von artefaktreichen Datensätzen sowie ein spezielles - analog zu bisher etablierten Programmen [Dunlop et al. 2006, Hahn et al. 2006, Victorino et al. 2004] - Programm zum Einteilen der Lunge in verschiedene speziell interessierende Areale (Regions of Interest) verdeutlichen den Bedarf und Nutzen neuer und für den Anwender leicht bedienbarer Software und Auswertungsmöglichkeiten, z.B. die direkte online-Analyse der gewonnenen Daten (siehe 4.3).

\subsection{Erörterung der Ergebnisse dieser Arbeit}

Die zentralen Fragen dieser Arbeit wurden durch aussagekräftige Ergebnisse beantwortet, es ergaben sich keine Widersprüche zwischen den durch die EIT 
erhobenen Daten und den klinischen Beobachtungen und Messungen. Herausragende Resultate waren dabei der Unterschied der relativen Impedanzänderung und damit der Ventilation zwischen maschineller Beatmung und Spontanatmung, der dabei beobachtete Effekt des Alters und der Thoraxcompliance auf die Ventilation, Unterschiede in der relativen Impedanzänderung in den ventralen und dorsalen Lungenbereichen sowie die Darstellung der Ventilationsverteilung unter Inhalationstherapie und verschiedenen Beatmungseinstellungen. Im Folgenden soll auf diese zentralen Ergebnisse und Beobachtungen näher eingegangen werden.

Bei der Betrachtung der Ergebnisse dieser Arbeit ist es wichtig zu beachten, dass in der Auswertung die Patienten auf der einen Seite als Gesamtkollektiv und auf der anderen Seite individuell berücksichtigt wurden.

\section{Durchführbarkeit der Studie}

Ein wesentliches Resultat dieser Studie ist, dass die EIT-Messungen auch an einem verhältnismäßig großen Patientenkollektiv unter normalen stationären Bedingungen auf einer pädiatrischen Intensivstation problemlos durchzuführen waren und aussagekräftige Daten lieferten. Sie störten den Stationsablauf nicht, zeigten keine relevanten Interferenzen mit anderen elektrischen Geräten auf der Intensivstation und wiesen den erwarteten signifikanten Unterschied der elektrischen Impedanzverteilung unter Beatmung und Spontanatmung auf. Dieser Unterschied hat besondere Bedeutung für die Nutzung der Methode als Monitoring der Lungenventilation und damit für die Überwachung und Optimierung der Beatmungstherapie, aber auch der weiteren Überwachung der Patienten nach der Extubation.

\section{Relative Impedanzänderung bei den Patienten während maschineller Beatmung und Spontanatmung}

Ein zentrales Ergebnis war die Korrelation der relativen Impedanzänderung mit der erwarteten Veränderung der Ventilation nach Extubation der Patienten. Diese zeigte sich sowohl bei Betrachtung des Gesamtdatensatzes als auch bei Betrachtung der Mittelwerte der jeweiligen Patienten. Insgesamt gesehen waren die Mittelwerte der relativen Impedanzänderung unter maschineller Beatmung signifikant höher als unter 
Spontanatmung (siehe Ergebnisse, 3.6.2), die Ventilation nahm entsprechend den Erwartungen nach der Extubation bei der Mehrzahl der Patienten deutlich ab (siehe Tab. 4 u. Abb. 23). Die Spontanatmung nach Extubation ist zumeist von höherer Frequenz und flacher, wobei der Narkoseüberhang, die atemdepressive Wirkung von sedierenden Medikamenten, postoperative Schmerzen, vermehrte Sekretbildung, inadäquater Hustensto $B$ atelektatische Lungenbereiche und eine Bronchialobstruktion eine wesentliche Rolle spielen. Zudem ist die Lungenfunktion vor allem in den ersten Tagen nach einem operativen Thoraxeingriff mit medianer Sternotomie im Sinne einer restriktiven Störung mit niedrigeren Tidalvolumina, einer erhöhten Resistance und einer verminderten funktionellen Residualkapazität (FRC) an sich schon deutlich eingeschränkt [Berrizbeita et al. 1988, Braun et al. 1978, Burgess et al. 1978, Estenne et al. 1985, Howatt et al. 1962, Locke et al. 1990, Polese et al. 1999, Stock et al. 1986].

Einige Patienten zeigten jedoch unmittelbar nach der Extubation ähnlich hohe oder höhere Werte der relativen Impedanzänderung als vor der Extubation. Dies lässt sich am ehesten dadurch erklären, dass die Extubation eine erhebliche Stresssituation für die Patienten darstellte. Im Gegensatz zu den oben genannten Ursachen einer schlechteren Ventilation nach Extubation kam es bei einigen Patienten aufgrund des Stresses vorübergehend zur Hyperventilation. Dieser Effekt war durch die EIT reproduzierbar darstellbar. Bei Patient 23 fiel nach der Extubation eine durch anhaltendes Weinen und Schluchzen bedingte Hyperventilation auf, was durch die erhöhten Werte der relativen Impedanzänderung gut abgebildet wurde.

Weiterhin bestand zwischen den Mittelwerten der relativen Impedanzänderung der jeweiligen Patienten vor und nach Extubation ein signifikanter positiver Zusammenhang unabhängig vom Alter: Je höher die Mittelwerte während der maschinellen Beatmung lagen, desto höher lagen sie auch unter Spontanatmung, wenn auch insgesamt auf niedrigerem Niveau. Verantwortlich für diesen Zusammenhang sind Lungengröße und Compliance sowie das damit einhergehende intrathorakale Gasvolumen. Eine gute Dehnbarkeit des Thorax sowie eine größere Lunge haben sowohl unter maschineller Beatmung als auch unter Spontanatmung ein größeres intrathorakales Gas volumen zur Folge als eine kleinere Lunge.

Der oben beschriebene deutliche Unterschied der relativen Impedanzänderung unter maschineller Beatmung und unter Spontanatmung war signifikant vom Alter der Patienten abhängig. Bei den Säuglingen unterschieden sich die Mittelwerte der 
relativen Impedanzänderungen unter maschineller Beatmung und unter Spontanatmung im Gegensatz zu den älteren Kindern (1-18 Jahre) nur wenig. Bei den älteren Kindern waren diese Unterschiede deutlich größer: Unter maschineller Beatmung wurden wesentlich höhere relative Impedanzänderungen gemessen als bei den Säuglingen. Allerdings zeigte die Altersgruppe der 1-3-jährigen Kinder tendenziell höhere relative Impedanzänderungen als die der 4-18-Jährigen. Gemäß der Annahme, dass die größeren Kinder auch die größere Thoraxcompliance haben [Oczenski 2008], wäre zu erwarten gewesen, dass die relativen Impedanzänderungen eigentlich bei den 4-18-Jährigen am größten sind. Bei Betrachtung der Patienten dieser Altersgruppe fiel jedoch auf, dass bei 7 der 16 Patienten eine Re-Operation am Thorax vorlag und die Mittelwerte unter maschineller Beatmung bei diesen 7 Patienten auch deutlich niedriger waren als bei den übrigen 9 Patienten dieser Altersgruppe mit erstmaligem Thoraxeingriff. Aufgrund von Voroperationen war die Compliance eingeschränkter [Kristjánsdóttir et al. 2004], dies liefert eine Erklärung der niedrigeren Mittelwerte der relativen Impedanzänderung bei den 4-18-Jährigen.

Die Entwicklung der Lunge mit der Ausreifung des Alveolarsystems, des Thorax, der Atemmuskulatur und damit der Atemmechanik ist ein dynamischer Prozess, der erst im frühen Erwachsenenalter bis zum 25. Lebensjahr vollständig abgeschlossen ist [Dunhill 1962, Gaultier 1995, Hislop und Reid 1981, Openshaw et al. 1984, Reid 1984, Thurlbeck 1975]. Der Thorax der Säuglinge besitzt anatomisch unter anderem aufgrund der nahezu horizontalen Rippenstellung eine niedrige Compliance und damit eine geringere Ausdehnungsmöglichkeit der Lunge [Muller und Bryan 1979, Openshaw et al. 1984, Richards und Bachmann 1961], was sich in den niedrigen Mittelwerten der relativen Impedanzänderung unter maschineller Beatmung niederschlägt. In den Mittelwerten der relativen Impedanzänderung zeigte sich im beatmeten Zustand eindeutig das Ausmaß der größeren Thoraxcompliance bei den älteren Kindern im Gegensatz zu den Säuglingen.

Interessanterweise unterschieden sich die Mittelwerte der relativen Impedanzänderung unter Spontanatmung zwischen den einzelnen Altersgruppen nur unwesentlich, die Ventilation fiel bei nahezu allen Patienten auf ein insgesamt ähnlich niedriges Niveau ab. Da der überwiegende Anteil der untersuchten Patienten aufgrund der postoperativen Schmerzen, der Thoraxdrainagen und der Sedativa unter Spontanatmung flach und mit hoher Frequenz atmete, kam hier das eben unter 
der maschinellen Beatmung beschriebene Phänomen der Thoraxcompliance und der daraus resultierenden höheren Tidalvolumina nicht zum Tragen.

\section{Unterschiede der relativen Impedanzänderung zwischen ventraler und dorsaler Lunge}

Die Ergebnisse der vorliegenden Studie an herzoperierten Kindern zeigten, dass die Mittelwerte der relativen Impedanzänderungen im ventralen Lungenbereich sowohl im beatmeten als auch im spontan atmenden Zustand signifikant höher waren als im dorsalen Lungenbereich, was auf eine stärkere Ventilation in den ventralen Lungenbereichen schließen lässt. Alle 29 Patienten befanden sich während der gesamten Untersuchung in Rückenlage, so dass die ventralen Lungenbereiche „oben" lagen und die dorsalen „unten“.

Allgemein ist bekannt, dass die regionale Ventilation bei gesunden Erwachsenen unter Spontanatmung abhängig von der Körperposition und der Schwerkraft ein inhomogenes Verteilungsmuster zeigt: Die jeweils unten liegenden Lungenbereiche weisen eine höhere regionale Ventilation auf als die oben liegenden [Bryan et al. 1966, Kaneko et al. 1966], welches durch EIT-Studien bestätigt werden konnte [Frerichs et al. 1996, 1998a, Hahn et al. 1996]. Auch eine neuere EI-Studie von Pham et al. (2011) an 32 gesunden spontan atmenden Neugeborenen in Rückenlage zeigte, dass die unten liegenden Lungenareale besser ventiliert waren als die oben liegenden. Damit konnten Pham et al. (2011) die oben genannten Ergebnisse bei Erwachsenen an den untersuchten Neugeborenen bestätigen. Ein weiterer Beleg gelang Humphreys et al. (2011) mittels einer EIT-Studie an 38 Säuglingen und Kindern mit einem angeborenen Herzfehler vor und während der Narkoseeinleitung mit Intubation und anschließender maschineller Beatmung: Unter Spontanatmung waren auch hier die unten liegenden Lungenareale besser ventiliert als die oben liegenden.

Unter maschineller Beatmung und Sedierung dagegen kommt es zu einer Beeinträchtigung der Lungenfunktion und zu einer Umkehrung des oben beschriebenen Befundes: Bei maschineller Inspiration wird das Zwerchfell passiv nach kaudal bewegt. Dies geschieht aufgrund des geringeren Widerstandes stärker im oben liegenden als im unten liegenden Lungenbereich. Somit werden die am wenigsten durchbluteten, oberen Lungenanteile vermehrt belüftet. Der Hauptanteil 
der Ventilation findet nun folglich in den sich besser ausdehnenden ventralen Lungenbereichen statt, während die der Unterlage aufliegenden dorsalen Lungenbereiche gemäß der Schwerkraft nicht so stark an der Ventilation beteiligt sind [Frerichs et al. 1998a, Froese und Bryan 1974, Humphreys et al. 2011, Victorino et al. 2004]. In der Studie von Frerichs et al. (1998a) handelte es sich um EITMessungen bei 10 Patienten, die sich einem operativen abdominellen Eingriff unterziehen mussten. Mittels EIT wurden präoperativ, während der intraoperativen Beatmung und nach Extubation die relativen Impedanzänderungen über dem Thorax gemessen. Unter Spontanatmung zeigte sich eine vermehrte Ventilation in den dorsalen Lungenbereichen, die sich unter maschineller Beatmung zugunsten der ventralen Lungenareale umkehrte und kurz nach Extubation und Rückkehr zur Spontanatmung wieder dem präoperativen Ventilationsmuster anglich. Die vorliegende Arbeit an herzoperierten Kindern bestätigte die oben genannten Ergebnisse an Erwachsenen in der Hinsicht, dass unter maschineller Beatmung in Rückenlage die ventralen Lungenareale besser belüftet sind als die dorsalen. Jedoch konnte, bezogen auf das Gesamtkollektiv, keine zunehmende Rekrutierung dorsaler Lungenareale nach der Extubation unter Spontanatmung beobachtet werden, wie dies in der Studie von Frerichs et al. (1998a) beschrieben wurde. Bei der Betrachtung der Profilkurven der untersuchten Patienten ist in einigen Fällen durchaus eine vermehrte Belüftung dorsaler Lungenareale nach Extubation zu sehen. Möglicherweise hätte ein weitaus längerer Messzeitraum nach dem Zeitpunkt der Extubation mehr Aufschluss darüber gegeben, ob und wann es bei den Kindern zu einer Rekrutierung der dorsalen Lungenanteile gekommen wäre. Andererseits zeigte die Studie von Frerichs et al. (1998a), dass sich die präoperativ herrschenden Ventilationsverhältnisse in den ersten Minuten bis Stunden nach Extubation nahezu wieder herstellten. Allerdings wurde bei den Patienten in der Studie von Frerichs ein abdominal-chirurgischer Eingriff (Laparoskopie oder Laparotomie) durchgeführt, während bei den Kindern in dieser Studie ein großer herzchirurgischer Eingriff durchgeführt wurde, der möglicherweise zu einer stärkeren und prolongierten Ausprägung der oben beschriebenen perioperativen Lungenfunktionsstörungen führte [Böhm und Bangert 2000, Gunnarsson et al. 1995, Putensen-Himmer et al. 1992]. Des Weiteren spielen Unterschiede in den Dosierungen der Sedativa und Analgetika eine große Rolle, da diese sich auf Atmung und Lungenfunktion auswirken und sich somit in der regionalen Ventilationsverteilung niederschlagen. 
Bisher ist über die regionale Ventilationsverteilung unter Spontanatmung und maschineller Beatmung sowie in verschiedenen Körperpositionen bei Kindern wenig publiziert worden, es existieren diesbezüglich vorwiegend Studien an Neugeborenen [Frerichs et al. 2003a, Heinrich et al. 2006, Pham et al. 2011, Schibler et al. 2009]. Die vorliegende Studie zeigt nun, dass sich die Rekrutierung dorsaler Lungenareale bei herzoperierten Patienten von der Rekrutierung bei gesunden Probanden bzw. bei Patienten nach extrathorakalen Eingriffen unterscheidet. In der oben genannten Studie von Humphreys et al. (2011) zeigte sich bei den Kindern nach Applikation der Narkotika und des Muskelrelaxans sowie mit dem Beginn der maschinellen Beatmung ebenfalls eine Umkehrung der Ventilationsverteilung: Die unter vorhergehender Spontanatmung gemessene vermehrte Ventilation in den unten liegenden (dorsalen) Lungenbereichen verschob sich zugunsten der oben liegenden (ventralen) Lungenbereiche. Damit stimmen die Ergebnisse der vorliegenden Arbeit unter maschineller Beatmung mit den Ergebnissen von Humphreys et al. (2011) überein.

In älteren Studien [Davies et al. 1985, Heaf et al. 1983] wurde postuliert, dass die regionale Ventilation bei Kindern - anders als bei Erwachsenen - in den unten liegenden Lungenarealen schlechter als in den oben liegenden ist. Allerdings waren die Kinder dieser beiden Studien im Gegensatz zu gesunden Erwachsenen in ihrer Lungenfunktion eingeschränkt und zumeist maschinell beatmet, was letztendlich doch die Tatsache bestätigt, dass unter Beatmung die ventralen Lungenareale besser belüftet werden.

Ein weiterer interessanter Punkt ist, dass bei den hier untersuchten Kindern der absolute Unterschied zwischen Beatmung und Spontanatmung in den ventralen Lungenbereichen größer als in den dorsalen Lungenbereichen war. Die Erklärung dafür liegt in den bereits oben beschriebenen Phänomenen der unterschiedlichen Ventilationsverteilung unter Beatmung und unter Spontanatmung: Im beatmeten Zustand sind die ventralen Lungenbereiche besser belüftet, im spontan atmenden Zustand die dorsalen Lungenbereiche. Da aber nach der Extubation eine gewisse Zeit vergeht bis die dorsalen Lungenbereiche rekrutiert sind und vermehrt an der Ventilation teilnehmen, erklärt sich der beobachtete größere absolute Unterschied zwischen Beatmung und Spontanatmung in den ventralen Lungenbereichen. 


\section{Zusammenhänge der relativen Impedanzänderung mit dem $\mathrm{p}_{\mathrm{a}} \mathrm{CO}_{2}$}

Die Mittelwerte des $\mathrm{p}_{\mathrm{a}} \mathrm{CO}_{2}$ zeigten unter der maschinellen Beatmung signifikant niedrigere Werte als unter Spontanatmung. Bei gleichzeitiger Betrachtung dieser Werte für die Phase der maschinellen Beatmung und für die Phase der Spontanatmung stellte sich ein negativer Zusammenhang zwischen dem $\mathrm{p}_{\mathrm{a}} \mathrm{CO}_{2}$ und der relativen Impedanzänderung dar: Die unter maschineller Beatmung gemessenen hohen Werte der relativen Impedanzänderung fielen mit niedrigen $\mathrm{p}_{\mathrm{a}} \mathrm{CO}_{2}$-Werten zusammen, während die unter Spontanatmung gemessenen niedrigeren relativen Impedanzänderungen mit höheren $\mathrm{p}_{\mathrm{a}} \mathrm{CO}_{2}$-Werten einhergingen. $\mathrm{Da}$ das im Blut gemessene $\mathrm{CO}_{2}$ ein $\mathrm{Maß}$ für die alveoläre Ventilation ist, unterstützt dieses Ergebnis die Hypothese, dass bei geringerer relativer Impedanzänderung die Ventilation auch insgesamt schlechter ist. Nach Beendigung der maschinellen Beatmung müssen die Patienten wieder ohne Unterstützung selbstständig atmen, was durch postoperative Schmerzen, die Thoraxdrainagen und einen Überhang an sedierenden und damit die Atemmechanik einschränkenden Medikamenten erheblich erschwert ist. Dieser Sachverhalt spiegelt sich in einer im Gegensatz zur maschinellen Beatmung geringeren Ventilation mit ansteigendem $\mathrm{p}_{\mathrm{a}} \mathrm{CO}_{2}$ wider. In einer tierexperimentellen Studie von Frerichs et al. (1998b) wurde in der linken Lunge von Schweinen mittels Aspiration von Ölsäure ein Atemnotsyndrom induziert. Wie in der vorliegenden Studie konnte in der Studie von Frerichs et al. (1998b) gezeigt werden, dass sich die im Blut gemessenen $\mathrm{CO}_{2}$-Werte vor chemischer Schädigung der Lunge signifikant von den Werten nach Schädigung unterschieden und dies mit den erhobenen EIT-Daten übereinstimmte.

\section{Darstellung der Wirkung einer Inhalationsbehandlung mit der EIT}

Für die Behandlung der Bronchialobstruktion gibt es heutzutage zahlreiche inhalativ einsetzbare bronchodilatatorisch wirksame Substanzen. Diese führen zu einer Erweiterung der Bronchien und folglich zu einer verbesserten Ventilation. Mussten Patienten nach der Extubation aufgrund einer Atemwegsobstruktion mit solchen bronchodilatatorisch wirksamen Substanzen (hier Salbutamol und Ipratropiumbromid) inhalativ behandelt werden, ließ sich der dadurch hervorgerufene Effekt mit der EIT durch eine Zunahme der relativen Impedanzänderung in bestimmten Lungenarealen 
reproduzierbar darstellen. Sobald die Inhalation beendet wurde, fiel die relative Impedanzänderung und damit die Ventilation dort wieder ab. Dies unterstreicht, dass die EIT-Methode Effekte einer im klinischen Altag gängigen Inhalationsbehandlung klar widerspiegelt und abbildet. Allerdings ist die hier beobachtete Zunahme der Ventilation unter Inhalation nicht unbedingt ausschließlich ein pharmakodynamischer Effekt der inhalierten Substanzen, sondern kann ebenso in der Compliance des Patienten begründet sein. Denn während der Inhalationsbehandlung versucht der Patient besonders auf seine Atmung zu achten und atmet deshalb tiefer und effektiver. Am ehesten liegt eine Kombination von pharmakologischer Wirkung der Substanzen und verbesserter Patientencompliance während der Inhalation vor.

\section{Darstellung von Beatmungsmanövern mit der EIT}

Die in dieser Studie beatmeten Kinder wurden mit den Beatmungsmodi SIMV, BIPAP sowie teilweise CPAP beatmet. SIMV und BIPAP sind Beatmungsmodi, bei denen dem Patienten zwischen kontrollierten Beatmungshüben durch den Respirator auch spontane Atemzüge möglich sind. Die SIMV ist eine volumen- oder druckkontrollierte Beatmung, bei der eine sequentielle Spontanatmung möglich ist, während die BIPAP-Beatmung druckkontrolliert ist und die Möglichkeit der simultanen ungehinderten Spontanatmung bietet. Bei dem CPAP-Verfahren atmet der Patient ausschließlich spontan, wird aber durch einen kontinuierlich positiven Atemwegsdruck unterstützt.

Gezielte Beatmungsmanöver (wie z.B. tierexperimentell bei Adler et al. 1998, Frerichs et al. 1999a, 2002, 2003b, 2007, Hinz et al. 2003b, Lindgren et al. 2007, Meier et al. 2008, Richard et al. 2009, van Genderingen et al. 2003, oder bei beatmeten Erwachsenen mit akutem Lungenversagen in den Studien von Costa et al. 2009 und Hinz et al. 2003a) waren im Rahmen dieser Arbeit aufgrund der klinischen Bedingungen nur sehr eingeschränkt möglich. Wurde der Beatmungsmodus von BIPAP auf CPAP umgestellt, kam es in den EIT-Messungen erwartungsgemäß zu einer Abnahme der relativen Impedanzänderung, da die CPAP Unterstützung auf einem deutlich niedrigeren Druckniveau stattfindet und der Patient ohne fest vorgegebene Frequenz alle Atemhübe selbst auslösen muss. Einer der Patienten (Patient 8) atmete kurz vor der Extubation nach Diskonnektion vom Beatmungsgerät spontan durch den Tubus; in der zugehörigen EIT-Messung war 
eine deutliche Abnahme der relativen Impedanzänderung zu verzeichnen. Eine mögliche Erklärung dafür ist der erhöhte Atemwegswiderstand infolge des Tubus, den der Patient aufgrund von mangelnder Kraft durch Medikamentenwirkungen und Schmerzen im Wundgebiet nicht suffizient überwinden konnte.

Somit wurden wie in vorangegangen Studien auch in dieser Studie Beatmungsmanöver und die daraus resultierenden Veränderungen in der relativen Thoraximpedanz über die EIT-Methode erkenn- und nachvollziehbar.

\section{Einfluss der Messdauer auf die Ergebnisse}

Bei 7 Patienten waren aufgrund von respiratorischer und/ oder kardiozirkulatorischer Instabilität und daraus resultierender prolongierter Beatmung Messungen über einen längeren Zeitraum (>24 Stunden) notwendig und mit der EIT möglich. Wenn sich der Elektroden-Haut-Kontakt im Verlauf der Messungen über mehrere Tage durch Schwitzen, Bewegungen, Umlagern und Waschen der Kinder verschlechterte und sich die Elektroden verschoben, wurden neue Elektroden an gleicher Thoraxstelle geklebt. Das Neukleben von Elektroden an gleicher Stelle ergibt nach Frerichs et al. (1998a), Marven et al. (1996), Reifferscheid et al. (2011) und Victorino et al. (2004) eine hohe Reproduzierbarkeit der Impedanzmessungen und kann deshalb im klinischen Alltag ohne Bedenken angewendet werden. Daraus erklärt sich das Ergebnis der vorliegenden Studie, dass die Messdauer die Variation der Impedanzänderung nicht beeinflusste und Messungen an einem Patienten über mehrere Tage mit der EIT problemlos möglich waren.

\section{Vergleich der Geschlechter und der beiden Lungenflügel}

Erwartungsgemäß ergaben sich im Gesamtkollektiv keine signifikanten Unterschiede der relativen Impedanzänderung zwischen den beiden Geschlechtern und zwischen rechtem und linkem Lungenflügel [Frerichs et al. 1996, Frerichs et al. 1999]. Bei einigen Patienten allerdings konnten im Verlauf der Untersuchungen Unterschiede in der Ventilation im rechten und linken Lungenflügel festgestellt werden. Darin zeigte sich, dass die Kinder individuell betrachtet unterschiedlich ventiliert waren. Mit den globalen Methoden zur Überwachung der Beatmung wie z.B. Blutgasanalysen und Messungen der transkutanen Sauerstoffsättigung können diese individuellen 
Inhomogenitäten in der Ventilation nicht dargestellt werden. Die EIT jedoch bringt eben diese Inhomogenitäten deutlich zum Ausdruck, so dass Unterschiede in der Belüftung der Lunge frühzeitig erkannt werden können und eindeutige Schlüsse bezüglich der Ventilationsverteilung unter Beatmung und ebenso unter Spontanatmung möglich sind. Folglich kann die Therapie für jeden Patienten individuell optimiert und angepasst werden.

\subsection{Anwendung der EIT bei herzoperierten Kindern}

Wie in der Literatur zumeist erwähnt, ist das Aufbauen des EIT-Systems einfach und schnell durchzuführen. Da es lediglich aus einem Notebook und dem nicht viel größeren EIT-Gerät besteht, ist es gut in Bettnähe unterzubringen und führt zu keinen Platzproblemen, was bei den engen räumlichen Verhältnissen und den vielen Geräten auf einer Intensivstation essentiell für die Nutzbarkeit im RoutineStationsablauf ist. Ist das System einmal aufgebaut und sind die Elektroden platziert, erfordern die Messungen keine Manipulation am Patienten mehr, sie können problemlos über längere Zeiträume fortgesetzt werden, ohne den Patienten zu stören. Diese Arbeit zeigte außerdem, dass es zu keinen relevanten Interferenzen zwischen dem EIT-System und den zahlreichen weiteren elektrischen Geräten auf der Intensivstation gekommen ist. Bei der Pflege der Patienten störten die Elektroden und die Kabel nicht, die therapeutischen und pflegerischen Routinemaßnahmen konnten ohne Einschränkungen durchgeführt werden. Als weiterer wichtiger Vorteil ist die Strahlendurchlässigkeit der Elektroden zu nennen: Bei dem Patientenkollektiv wurden regelmäßig Röntgenaufnahmen des Thorax durchgeführt, die somit kein Entfernen und Neukleben der Elektroden erforderten.

Das übliche Kleben der 16 Elektroden zirkulär um den Thorax dauerte mit etwa 10-15 Minuten relativ lange; ein Problem bei den herzoperierten Kindern war zudem der geringe Platz auf dem Thorax durch das Pflaster über der medianen Sternotomie, die Wunddrainagen, die externen Schrittmacherkabel und die zur Überwachung notwendigen EKG-Elektroden. Vor allem bei den kleineren Kindern und den Säuglingen war das Kleben der Elektroden aufgrund des geringeren Thoraxumfanges noch schwieriger, durch Zuschneiden der Elektroden war dieses Problem jedoch immer lösbar. Um die Elektroden am Rücken platzieren zu können, 
war stets mindestens eine Hilfsperson notwendig, da das Drehen der Kinder aufgrund der vielen Kabel, Drainagen und Zugänge für eine einzelne Person nicht möglich war. Bei Messungen, die über mehrere Tage erfolgten, war teilweise das Neukleben einiger Elektroden nötig, da diese durch das Schwitzen und die Bewegungen der Kinder sowie durch pflegerische Maßnahmen wie Umlagern oder Waschen irgendwann nicht mehr ausreichend hafteten. Sobald die Patienten nach Reduzierung der Sedativa wacher wurden, zappelten und/ oder weinten sie häufig, was vor allem bei den Säuglingen der Fall war. Dies erschwerte die Messungen durch einen verschlechterten Elektroden-Haut-Kontakt und Bewegungsartefakte.

Aus den erwähnten Erfahrungen in der Anwendung der EIT auf der pädiatrischen Intensivstation ergeben sich folgende Schlussfolgerungen und Vorschläge für die Praxis:

- Die Befestigung der Elektroden über ein Gummiband/ einen Gürtel auch für den pädiatrischen Bereich wäre wünschenswert, damit der ElektrodenHautkontakt auch über lange Messzeiträume konstant ist und der relativ große Zeitaufwand des Elektrodenklebens entfällt.

- Es wäre zu untersuchen und zu prüfen, ob auch mit weniger Elektroden ausreichend gute Messergebnisse erzielt werden könnten. Dadurch wäre das Platzproblem auf dem Thorax wesentlich geringer.

- Um neben den vielen, ohnehin schon vorhandenen Kabeln auf einer Intensivstation die 16 zu den jeweiligen Elektroden führenden Kabel zu reduzieren, wäre eine kabellose Verbindung wünschenswert.

- Die offline-Aufbereitung der Daten ist noch zu aufwendig und dauert zu lange (Verwerfen von komplett gestörten Datensätzen, Entfernen von gestörten Sequenzen in Datensätzen, Erstellen von Profilkurven zur Verlaufsbeurteilung). Der Arzt benötigt jedoch die Daten so schnell wie möglich direkt am Krankenbett, um die Therapie optimieren und anpassen zu können. Hierzu ist eine Weiterentwicklung und Automatisierung der Software erforderlich.

- Eine unmittelbare online-Analyse der gewonnenen EIT-Daten ist anzustreben, um direkte Schlüsse und mögliche Konsequenzen der Therapie aus den Ergebnissen ziehen zu können. 


\subsection{Schlussfolgerungen und Ausblick}

In dieser Arbeit konnte gezeigt werden, dass die EIT als neue nicht-invasive, strahlungsfreie Methode zur Beurteilung der regionalen Lungenventilation auf einer pädiatrischen Intensivstation bei Kindern nach operativem Thoraxeingriff problemlos anwendbar ist und eine sinnvolle Ergänzung zu den routinemäßig erhobenen Parametern zur Überwachung der pulmonalen Funktion und Belüftungssituation darstellt.

Die Beurteilung und Auswertung von EIT-Daten ist aussagekräftiger bei Betrachtung eines einzelnen Patienten als bei Betrachtung eines gesamten Kollektivs. Jeder Patient an sich ist durch so viele individuelle Gegebenheiten charakterisiert, die unter der speziellen Beatmungstherapie ( $\mathrm{FiO}_{2}$, Inspirationsdruck, Medikamente) nochmals modifiziert werden. Daher kann der Vergleich eines Gesamtkollektivs nur Tendenzen hinsichtlich der relativen Impedanzänderung und Ventilationsverteilung aufzeigen; die individuellen Charakteristika eines jeden Patienten gehen dabei verloren. Die EIT ist in der Praxis zur Beurteilung der Ventilationssituation innerhalb einzelner Patienten zur Therapieanpassung und -optimierung einsetzbar; diese Arbeit bestätigt eindeutig die Möglichkeiten, die die EIT dabei direkt am Krankenbett liefern kann.

Trotz intensiver Fortschritte in der EIT-Forschung besteht aber weiterhin ein erheblicher Forschungsbedarf zur weiteren Optimierung dieser Methode und deren klinischer Anwendung im pädiatrischen Bereich. 


\section{Zusammenfassung}

In der vorliegenden Arbeit wurde die regionale Lungenventilation von Kindern mit einem angeborenen Herzfehler nach einer Herzoperation mittels der Elektrischen Impedanztomographie (ET) untersucht. Dabei waren die Patienten postoperativ zunächst maschinell beatmet, bis sie im Verlauf extubiert werden konnten. Das Kollektiv umfasste 30 Kinder beiderlei Geschlechts im Alter von 0-18 Jahren. Die Untersuchung fand unter normalen klinischen Bedingungen auf der pädiatrischen Intensivstation statt, und die Anwendbarkeit der EIT wurde im Rahmen der alläg lichen stationären Gegebenheiten geprüft und beurteilt.

Die EIT besitzt als bildgebendes Verfahren das Potenzial, anhand von Spannungsmessungen an der Oberfläche des Thorax Rückschlüsse auf die regionale Verteilung der Impedanz im Thoraxinneren und damit auf die Ventilation zu ziehen. Im Rahmen einer Beatmungstherapie ist die Darstellung der regionalen Ventilationsverhältnisse von Bedeutung, um die Beatmung an die jeweiligen Bedürfnisse des Patienten individuell besser anpassen zu können und somit dem Ziel einer lungenprotektiven Beatmung gerecht zu werden und damit der Lunge und dem Herz-Kreislaufsystem mit der Beatmung so wenig wie möglich zu schaden.

Zusammenfassend können folgende wesentliche Ergebnisse der EIT-Studie formuliert werden:

Die EIT war problemlos auf einer pädiatrischen Intensivstation anwendbar und lieferte bei 29 von 30 Patienten aussagekrättige Ergebnisse. Dabei wurden der allägliche Stationsablauf sowie die Routineversorgung nicht gestört. Zudem traten keine wesentlichen Störungen zwischen dem EIT-Gerät und den zahlreichen weiteren Geräten auf der Intensivstation auf.

Zwischen Beatmung und Spontanatmung zeigte sich in der vorliegenden Studie die erwartete signifikante altersabhängige Abnahme der relativen Impedanzänderung und somit der Ventilation.

Der ventrale Lungenbereich war sowohl unter Beatmung als auch unter Spontanatmung besser ventiliert als der dorsale Lungenbereich; unter Spontanatmung allerdings fiel der Unterschied geringer aus. 
Bei den Kindern nach einer Re-Operation wurden niedrigere relative Impedanzänderungen gemessen als bei den Kindern nach primärer Operation.

Zwischen dem $\mathrm{p}_{\mathrm{a}} \mathrm{CO}_{2}$ und der relativen Impedanzänderung zeigte sich ein negativer Zusammenhang: Die unter maschineller Beatmung gemessenen hohen Werte der relativen Impedanzänderung fielen mit niedrigen $\mathrm{p}_{\mathrm{a}} \mathrm{CO}_{2}$-Werten zusammen, während die unter Spontanatmung gemessenen niedrigeren relativen Impedanzänderungen mit höheren $\mathrm{p}_{\mathrm{a}} \mathrm{CO}_{2}$-Werten einhergingen.

Effekte einer Inhalationsbehandlung ließen sich mit der EIT reproduzierbar abbilden.

Die Unterschiede in der Ventilation bei verschiedenen Beatmungsmodi (SIMV, BIPAP, CPAP) wurden mit der EIT nachvollziehbar erfasst.

Die Studie bestätigt eindeutig, dass die EIT im klinischen Alltag neben den Routineparametern eine sinnvolle und bedeutende Ergänzung zur Überwachung und Beurteilung einer Beatmungstherapie darstellt. Diese Methode ist in ihrer Anwendung unkompliziert und schmerzfrei, was gerade in der Pädiatrie eine bedeutende Rolle spielt. Gemäß den Erwartungen spiegeln sich die Unterschiede in der Ventilation zwischen maschineller Beatmung und Spontanatmung, zwischen verschiedenen Beatmungsmodi, bei Inhalationsbehandlungen sowie bei Betrachtung der ventralen und dorsalen Lungenbereiche wider und werden deutlich und nachvollziehbar von der EIT abgebildet.

Um weitere Fortschritte in der klinischen Einsatzfähigkeit der EIT zu erzielen und die Methode in Zukunft zunehmend zu etablieren, ist die weitere Vereinfachung des Auswertungsprozesses der gewonnenen Daten notwendig; die Ergebnisse sollten in Echtzeit direkt am Krankenbett verfügbar sein, damit daraus sofortige Konsequenzen für die Therapie gezogen werden können. 


\section{Anhang}

\section{Patientendaten}

\begin{tabular}{|c|c|c|c|c|c|c|}
\hline Pat. & Geschl. & $\begin{array}{l}\text { Alter } \\
\text { (Jahre) }\end{array}$ & $\begin{array}{l}\text { Größe } \\
\text { (cm) }\end{array}$ & $\begin{array}{l}\text { Gewicht } \\
\text { (kg) }\end{array}$ & $\begin{array}{c}\text { Diagnose } \\
\text { (Art des Herzfehlers) }\end{array}$ & Operation aktuell \\
\hline 1 & $\mathrm{~m}$ & $\begin{array}{c}11 \\
6 / 12\end{array}$ & 151 & 32,7 & $\begin{array}{l}\text { Fallot'sche Tetralogie; Z.n. } \\
\text { Korrektur-OP mit klappen- } \\
\text { tragendem Conduit u. trans- } \\
\text { anulärer Ausflussbahnplastik } \\
\text { mittels Patch 02/96; Z.n. } \\
\text { Direktverschluss } 3 \text { kleiner } \\
\text { VSD’s 10/96; Aneurysma der } \\
\text { linken PA; Verkalkung der } \\
\text { Conduitklappe mit hochgradi- } \\
\text { ger Conduit-Stenose u. post- } \\
\text { stenotisches Aneurysma bis } \\
\text { in die LPA; Z.n. Dilatation der } \\
\text { Conduit-Stenose mit Ballon } \\
\text { 05/04 }\end{array}$ & $\begin{array}{l}\text { Korrektur-OP mit Aus- } \\
\text { tausch der Pulmonal- } \\
\text { klappenprothese, Ersatz } \\
\text { des Pulmonalarterien- } \\
\text { truncus u. der proximalen } \\
\text { LPA mit Pulmonalishomo- } \\
\text { graft }\end{array}$ \\
\hline 2 & $m$ & $\begin{array}{c}13 \\
0 / 12\end{array}$ & 166 & 55,5 & $\begin{array}{l}\text { postduktale Aortenisthmus- } \\
\text { stenose; mittelgradige } \\
\text { Aortenklappenins uffizienz }\end{array}$ & $\begin{array}{l}\text { Korrektur mit ISTA- } \\
\text { Resektion und schräger } \\
\text { End-zu-End-Anastomose }\end{array}$ \\
\hline 3 & $\mathrm{~m}$ & $\begin{array}{c}5 \\
7 / 12\end{array}$ & 103 & 17,4 & $\begin{array}{l}\text { Fallot'sche Tetralogie mit } \\
\text { infundibulärer Pulmonal- } \\
\text { stenose u. hochgradige LPA- } \\
\text { Stenose; links perstistierende } \\
\text { obere Hohlvene }\end{array}$ & $\begin{array}{l}\text { Korrektur mit Aorten- } \\
\text { homograft; Resektion der } \\
\text { Bifurkation u. semizirku- } \\
\text { läre End-zu-End-Anasto- } \\
\text { mose }\end{array}$ \\
\hline 4 & $\mathrm{~m}$ & $\begin{array}{c}17 \\
5 / 12\end{array}$ & 179 & 66,7 & $\begin{array}{l}\text { kombiniertes Aortenvitium mit } \\
\text { mittelgroßer Stenose und } \\
\text { hochgradiger Insuffizienz }\end{array}$ & $\begin{array}{l}\text { Ross-Operation mit PA- } \\
\text { Homograft }\end{array}$ \\
\hline 5 & w & $\begin{array}{c}3 \\
2 / 12\end{array}$ & 100 & 14,5 & ASD II; relative PS & $\begin{array}{l}\text { ASD-Verschluss mittels } \\
\text { Perikardpatch }\end{array}$ \\
\hline 6 & $\mathrm{w}$ & $\begin{array}{c}3 \\
3 / 12\end{array}$ & 93,5 & 13,3 & $\begin{array}{l}\text { großer muskulärer apikaler } \\
\text { VSD; großer ASD II; valvu- } \\
\text { läre PS;pulmonale Hyper- } \\
\text { tonie }\end{array}$ & $\begin{array}{l}\text { VSD-Verschluss mittels } \\
\text { Dacron-Patch; ASD- } \\
\text { Direkt verschluss }\end{array}$ \\
\hline 7 & $\mathrm{w}$ & $\begin{array}{c}0 \\
11 / 12\end{array}$ & 63 & 6,8 & $\begin{array}{l}\text { DORV; absent pulmonary } \\
\text { valve syndrom mit dys- } \\
\text { plastischer Pulmonalklappe } \\
\text { u. signifikanter Herzinsuffi- } \\
\text { zienz; VSD; Z.n. VSD- } \\
\text { Patchverschluss u. Implan- } \\
\text { tation eines RV-PA-Conduits } \\
\text { 04/04; art. Hypertonie; } \\
\text { Dystelektase des linken } \\
\text { Mittellappens }\end{array}$ & $\begin{array}{l}\text { Conduit-Austausch bei } \\
\text { Conduit-Stenose u. Con- } \\
\text { duit-Insuffizienz }\end{array}$ \\
\hline 8 & $\mathrm{~m}$ & $\begin{array}{c}7 \\
5 / 12\end{array}$ & 118 & 21,6 & $\begin{array}{l}\text { Shone-Komplex; Z.n. Resek- } \\
\text { tion einer Aortenisthmus- } \\
\text { stenose mit schräger End-zu- } \\
\text { End-Anastomose 01/97; } \\
\text { mittelgradige Subaorten- } \\
\text { stenose }\end{array}$ & $\begin{array}{l}\text { Resektion der Sub- } \\
\text { aortenstenose mittels } \\
\text { Bigelow-Inzision }\end{array}$ \\
\hline 9 & $\mathrm{~m}$ & $\begin{array}{c}3 \\
3 / 12\end{array}$ & 105 & 16,2 & $\begin{array}{l}\text { Sinus-Venosus-ASD u. } \\
\text { partielle Lungenvenenfehl- } \\
\text { mündung }\end{array}$ & ASD-Patchverschluss \\
\hline 10 & $\mathrm{~m}$ & $\begin{array}{c}0 \\
4 / 12\end{array}$ & 61 & 4,3 & kompletter AVSD & $\begin{array}{l}\text { Korrektur des AVSD } \\
\text { mittels Perikardpatch }\end{array}$ \\
\hline
\end{tabular}




\begin{tabular}{|c|c|c|c|c|c|c|}
\hline 11 & $\mathrm{~m}$ & $\begin{array}{c}7 \\
0 / 12\end{array}$ & 115 & 19,7 & $\begin{array}{l}\text { Fallot'sche Tetralogie; Z.n. } \\
\text { Ballonvalvuloplastie der } \\
\text { Pulmonalklappe 04/98; Z.n. } \\
\text { Korrektur-OP mit VSD-Patch- } \\
\text { Verschluss und Erweiterung } \\
\text { des rechts ventrikulären } \\
\text { Ausflusstraktes mit autolo- } \\
\text { gem Perikard ohne trans- } \\
\text { anuläre Patchplastik 11/98 }\end{array}$ & $\begin{array}{l}\text { Pulmonalklappenersatz } \\
\text { mittels Homograft bei } \\
\text { bedeutsamer Pulmonal- } \\
\text { klappeninsuffizienz }\end{array}$ \\
\hline 12 & $\mathrm{w}$ & $\begin{array}{c}5 \\
9 / 12\end{array}$ & 119 & 21,9 & $\begin{array}{l}\text { Truncus arteriosus commu- } \\
\text { nis Typ I; Z.n. Korrektur-OP } \\
\text { mit klappentragendem Con- } \\
\text { duit 10/ 99; erhöhter rechts- } \\
\text { ventrikulärer Druck bei höher- } \\
\text { gradiger Conduit-Stenose; } \\
\text { geringradige Conduit-Insuffi- } \\
\text { zienz; Abgangsstenose der } \\
\text { LPA; mittelgradige Mitral- } \\
\text { klappeninsuffizienz }\end{array}$ & $\begin{array}{l}\text { Conduit-Austausch gegen } \\
\text { Pulmonalis-Homograft; } \\
\text { Erweiterungsplastik der } \\
\text { LPA mit Homograft- } \\
\text { Anastomose; Inspektion } \\
\text { der Mitralklappe trans- } \\
\text { atrioseptal }\end{array}$ \\
\hline 13 & $\mathrm{w}$ & $\begin{array}{c}3 \\
0 / 12\end{array}$ & 92,5 & 12 & $\begin{array}{l}\text { ASD II; relative PS; } \\
\text { Mitralinsuffizienz ol }\end{array}$ & $\begin{array}{l}\text { ASD-Verschluss mittels } \\
\text { Perikardpatch }\end{array}$ \\
\hline 14 & $\mathrm{w}$ & $\begin{array}{c}9 \\
3 / 12\end{array}$ & 137 & 39,7 & $\begin{array}{l}\text { d-TGA; links persistierende } \\
\text { obere Hohlvene; Z.n. art. } \\
\text { Switch-OP 02/ 96; post- } \\
\text { operative Myokardischämie } \\
\text { bei Verschluss der Art. cir- } \\
\text { cumflexa sin.; Z. n. Bypass- } \\
\text { OP der linken Art. mammaria } \\
\text { interna auf den Ramus cir- } \\
\text { cumflexus bei Stenose der } \\
\text { linken Coronararterie 07/02; } \\
\text { mittelgradige Al; proximale } \\
\text { Stenose des Ramus interven- } \\
\text { trikularis ant.; Abfluss des } \\
\text { Coronarsinus in den li Vorhof; } \\
\text { Stenose des Coronarsinus }\end{array}$ & $\begin{array}{l}\text { Aortenklappenersatz; } \\
\text { Unroofing des Koronar- } \\
\text { sinus; Ligatur der links } \\
\text { persistierenden oberen } \\
\text { Hohlvene sowie Ligatur } \\
\text { der V. hemiazygos }\end{array}$ \\
\hline 15 & $\mathrm{~m}$ & $\begin{array}{c}6 \\
0 / 12\end{array}$ & 115 & 20 & $\begin{array}{l}\text { Fallot-Tetralogie mit Pulmo- } \\
\text { nalklappenagenesie; Zn. } \\
\text { Korrektur-OP mit klappen- } \\
\text { tragendem Conduit und VSD- } \\
\text { Verschluss; Infundibulekto- } \\
\text { mie; Reduktionsplastik beider } \\
\text { PA; PFO-Direktverschluss } \\
\text { 12/ 99; Conduit-Stenose und } \\
\text {-Insuffizienz }\end{array}$ & Conduitaustausch \\
\hline 16 & $\mathrm{~m}$ & $\begin{array}{c}0 \\
3 / 12\end{array}$ & 57 & 3,95 & $\begin{array}{l}\text { perim embranöser VSD; } \\
\text { ASD II }\end{array}$ & $\begin{array}{l}\text { VSD-Patch-Verschluss; } \\
\text { ASD II-Direkt verschluss; } \\
\text { Reins ertion des Trikus- } \\
\text { pidalklappensegels }\end{array}$ \\
\hline 17 & $\mathrm{~m}$ & $\begin{array}{c}1 \\
0 / 12\end{array}$ & 75 & 9,0 & $\begin{array}{l}\text { DORV; großer VSD; d-MGA; } \\
\text { bilateraler Konus; geringe } \\
\text { supravalvuläre Aortenenge; } \\
\text { Aortenbogen distal hypo- } \\
\text { plastisch mit diskreter } \\
\text { Isthmusstenose; ASD II; Z.n. } \\
\text { PA-Banding, ISTA-Resektion, } \\
\text { Aortenbogenplastik u. } \\
\text { Atrioseptektomie 06/ 04; } \\
\text { Damus-Kaye-Stensel-OP; } \\
\text { Debanding; modifizierter BTA } \\
\text { rechts }\end{array}$ & $\begin{array}{l}\text { Anlage einer } \\
\text { bidirektionalen Glenn- } \\
\text { Anastomose sowie } \\
\text { Erweiterungsplastik der } \\
\text { linken PA }\end{array}$ \\
\hline
\end{tabular}




\begin{tabular}{|c|c|c|c|c|c|c|}
\hline 18 & $\mathrm{w}$ & $\begin{array}{c}7 \\
1 / 12\end{array}$ & 129 & 29,0 & $\begin{array}{l}\text { kombiniertes Aortenvitium mit } \\
\text { membranöser subvalvulärer } \\
\text { AS und geringgradiger AI }\end{array}$ & $\begin{array}{l}\text { Resektion der } \\
\text { subvalvulären AS }\end{array}$ \\
\hline 19 & $\mathrm{~m}$ & $\begin{array}{c}7 \\
10 / 12\end{array}$ & 120 & 26,0 & $\begin{array}{l}\text { hochgradige valvuläre AS; } \\
\text { gering gradige supraval vuläre } \\
\text { AS; mittelgradige Aorten- } \\
\text { isthmusstenose; mittelgradige } \\
\text { Aortenklappenins uffizienz; } \\
\text { Abgangsstenosen beider PA }\end{array}$ & $\begin{array}{l}\text { Kommissurotomie der } \\
\text { Aortenklappe mit ISTA- } \\
\text { Resektion }\end{array}$ \\
\hline 20 & $\mathrm{~m}$ & $\begin{array}{c}5 \\
10 / 12\end{array}$ & 118,5 & 20 & $\begin{array}{l}\text { Mitralinsuffizienz bei } \\
\text { Fenestration des posterioren } \\
\text { Mitralsegels }\end{array}$ & $\begin{array}{l}\text { Verschluss der Fenes- } \\
\text { tration; Mitralklappen- } \\
\text { rekonstruktion }\end{array}$ \\
\hline 21 & $\mathrm{~m}$ & $\begin{array}{c}2 \\
5 / 12\end{array}$ & 95 & $\overline{16,4}$ & Sinus-Venosus-ASD & $\begin{array}{l}\text { Korrektur des Sinus- } \\
\text { Venosus-Defektes mittels } \\
\text { Perikardpatch }\end{array}$ \\
\hline 22 & $\mathrm{~m}$ & $\begin{array}{c}0 \\
4 / 12\end{array}$ & 58 & 5,02 & perimembranöser VSD & VSD-Verschluss \\
\hline 23 & $\mathrm{~m}$ & $\begin{array}{c}0 \\
2 / 12\end{array}$ & 52 & 3,6 & mehrere VSD's; ASD & PA-Banding \\
\hline 24 & $\mathrm{~m}$ & $\begin{array}{c}5 \\
4 / 12\end{array}$ & 116 & 28,0 & $\begin{array}{l}\text { Fallot'sche Tetralogie; Z.n. } \\
\text { interventionellem Verschluss } \\
\text { einer aortopulmonalen } \\
\text { Kollaterale 06/01; Z.n. } \\
\text { Korrektur-OP mit transanu- } \\
\text { lärer Patch-Plastik 08/01 }\end{array}$ & $\begin{array}{l}\text { Conduit-Implantation } \\
\text { (klappentragender Homo- } \\
\text { graft) in Pulmonalisposi- } \\
\text { tion }\end{array}$ \\
\hline 25 & $\mathrm{~m}$ & $\begin{array}{c}6 \\
0 / 12\end{array}$ & 113 & 22,4 & membranöser VSD & VSD-Direktverschluss \\
\hline 26 & $\mathrm{~m}$ & $\begin{array}{c}1 \\
2 / 12\end{array}$ & 68 & 5,0 & VSD & $\begin{array}{l}\text { VSD-Verschluss mit } \\
\text { Patch }\end{array}$ \\
\hline 27 & w & $\begin{array}{c}15 \\
8 / 12\end{array}$ & 161 & 61 & $\begin{array}{l}\text { PA- und PA-Bifurkations- } \\
\text { stenose; VSD; Z.n. PA- } \\
\text { Erweiterungsplastik und ZAP- } \\
\text { Shunt 05/90; Z.n. atyp. BTA } \\
\text { links 09/92; Z.n. Korrektur-OP } \\
\text { mit Conduit 07/94 }\end{array}$ & $\begin{array}{l}\text { Conduit-Austausch gegen } \\
\text { Pulmonalishomograft }\end{array}$ \\
\hline 28 & $\mathrm{~m}$ & $\begin{array}{c}0 \\
3 / 12\end{array}$ & 65 & 6,5 & großer VSD; PFO & $\begin{array}{l}\text { VSD-Patchverschluss; } \\
\text { Direktverschluss des PFO }\end{array}$ \\
\hline 29 & w & $\begin{array}{c}6 \\
5 / 12\end{array}$ & 120,5 & 21,8 & VSD; PFO & $\begin{array}{l}\text { VSD- und PFO-Direkt- } \\
\text { verschluss }\end{array}$ \\
\hline 30 & $\mathrm{w}$ & $\begin{array}{c}11 \\
4 / 12\end{array}$ & 139 & 31 & $\begin{array}{l}\text { kompletter AVSD; Mitralkleft } \\
\text { mit geringer MI }\end{array}$ & $\begin{array}{l}\text { AVSD-Direktverschluss; } \\
\text { Direkt verschluss des } \\
\text { Spaltes im septalen Segel } \\
\text { der Mitralklappe }\end{array}$ \\
\hline
\end{tabular}




\section{Literaturverzeichnis}

Adler A, Amyot R, Guardo R, Bates JH, Berthiaume Y (1997):

Monitoring changes in lung air and liquid volumes with electrical impedance tomography. J Appl Physiol 83, 1762-1767

Adler A, Shinozuka N, Berthiaume Y, Guardo R, Bates JH (1998):

Electrical impedance tomography can monitor dynamic hyperinflation in dogs. J Appl Physiol 84, 726-732

Amato MBP, Barbas CSV, Medeiros DM, Magaldi RB, Schettino GPP, Lorenzi-Filho G, Kairalla RA, Deheinzelin D, Munoz C, Oliveira R, Takagaki TY, Carvalho CRR (1998):

Effect of a protective ventilation strategy on mortality in acute respiratory distress syndrome. N Engl J Med $\underline{338}, 347-354$

Baisch F, Hahn G, Sipinkova I, Beer M, Hellige G (1995):

Comparison of electrode belts with "spot" electrodes for electrical impedance tomography. Innov Tech Biol Med 16, 119-125

Barber DC (1990):

Quantification in impedance imaging. Clin Phys Physiol Meas 11, Suppl A, 4556

Barber DC, Brown BH (1984):

Applied potential tomography. J Phys E Sci Instrum 17, 723-733

Berrizbeita LD, Tessler S, Lenora RAK (1988):

Effect of sternotomy and coronary bypass surgery on postoperative pulmonary mechanics. Am Rev Respir Dis 137, 248

Bikker IG, Leonhardt S, Bakker J, Gommers D (2009):

Lung volume calculated from electrical impedance tomography in ICU patients at different PEEP levels. Intensive Care Med $\underline{35}$, 1362-1367 
Bikker IG, Preis C, Egal M, Bakker J, Gommers D (2011):

Electrical impedance tomography measured at two thoracic levels can visualize the ventilation distribution changes at the bedside during a decremental positive end-expiratory lung pressure trial. Crit Care 15, R 193

Böhm SH, Bangert K (2000):

Prävention und Therapie anästhesiebedingter Atelektasen. Anästhesist $\underline{49}$, 345-34

Braun SR, Birnbaum ML, Chopra PS (1978):

Pre- and postoperative pulmonary function abnormalities in coronary artery revasculation surgery. Chest $\underline{73}, 316-320$

Brown BH, Barber DC (1987):

Electrical impedance tomography; the construction and application to physiological measurement of electrical impedance images. Med Prog Tech $\underline{13}$, 69-75

Brown BH, Seagar AD (1987):

The Sheffield data collection system. Clin Phys Physiol Meas $\underline{8}$, Suppl A, 91-97

Brown BH, Barber DC, Seagar AD (1985):

Applied potential tomography: possible clinical applications. Clin Phys Physiol Meas $\underline{6}, 109-121$

Brown BH, Primhak RA, Smallwood RH, Milnes P, Narracott AJ, Jackson MJ (2002): Neonatal lungs - can absolute lung resistivity be determined non-invasively? Med Biol Eng Comput 40, 388-394

Bryan AC., Milic-Emili J., Pengelly D. (1966):

Effect of gravity on the distribution of pulmonary ventilation. J Appl Phys $\underline{21}$, 778-784 
Burgess GE, Cooper JR, Marino RJ (1978):

Pulmonary effect of pleurotomy during and after coronary artery surgery with internal mammary artery versus saphenous vain grafts. J Thorac Cardiovasc Surg $\underline{76}, 230-234$

Campbell JH, Harris ND, Zhang F, Brown BH, Morice AH (1994):

Clinical applications in the monitoring of changes in intrathoric fluid volumes. Physiol Meas $\underline{15}, 217-222$

Caples SM, Hubmayr RD (2003):

Respiratory monitoring tools in the intensive care unit. Curr Opin Crit Care $\underline{9}$, 235-235

Costa EL, Borges JB, Melo A, Suarez-Sipmann F, Toufen C Jr, Bohm SH, Amato MB (2009):

Bedside estimation of recruitable alveolar collapse and hyperdistension by electrical impedance tomography. Intensive Care Med $\underline{35}$, 1132-1137

Dalton ML, Connally SR (1993):

Median sternotomy. Surg Gynecol Obstet 176, 615-624

Davies H, Kitchman R, Gordon I, Helms P (1985):

Regional ventilation in infancy. Reversal of adult pattern. N Engl J Med $\underline{26}$, 1626-1628

Dittmar J, Just A, Hellige G, Quintel M, Hahn G (2010):

Ein Softwarepaket zur Auswertung von Messserien der Elektrischen Impedanztomographie (EIT). Biomed Tech 55, 39-42

Dunhill MS (1962):

Postnatal growth of the lung. Thorax $\underline{17}, 329-333$ 
Dunlop S, Hough J, Riedel T, Fraser JF, Dunster K, Schibler A (2006):

Electrical impedance tomography in extremely prematurely born infants and during high frequency oscillatory ventilation analyzed in the frequency domain. Physiol Meas $\underline{27}, 1151-1165$

Erlandsson K, Odenstedt H, Lundin S, Stenquist O (2006):

Positive end-expiratory pressure optimization using electric impedance tomography in morbidly obese patients during laparoscopic gastric bypass surgery. Acta Anaesthesiol Scand $\underline{50}, 833-839$

Estenne M, Yernault JC, De Smet JM, De Troyer A (1985):

Phrenic and diaphragm function after bypass grafting. Thorax $\underline{40}, 293-299$

Eyüboglu BM, Öner AF, Baysal U, Biber C, Keyf AI, Yilmaz Ü, Erdogan Y (1995):

Application of electrical imepdance tomography in diagnosis of emphysema - a clinical study. Physiol Meas $\underline{16}, 191-211$

Frerichs I, Hahn G, Hellige G (1996):

Gravity-dependent phenomena in lung ventilation determined by functional EIT. Physiol Meas $\underline{17}, 149-157$

Frerichs I, Hahn G, Golisch W, Kurpitz M, Burchardi H, Hellige G (1998a):

Monitoring perioperative changes in distribution of pulmonary ventilation by functional electrical impedance tomography. Acta Anaesthesiol Scand $\underline{42}, 721$ 726

Frerichs I, Hahn G, Schröder T, Hellige G (1998b):

Electrical impedance tomography in monitoring experimental lung injury. Intensive Care Med 24

Frerichs I, Hahn G, Hellige G (1999a):

Thoracic electrical impedance tomographic measurements during volume controlled ventilation - effects of tidal volume and positive end-expiratory pressure. IEEE Trans Med Imaging 18, 764-773 
Frerichs I, Hahn G, Schiffmann H, Berger C, Hellige G (1999b):

Monitoring regional lung ventilation by functional electrical impedance tomography during assisted ventilation. Ann N Y Acad Sci $\underline{873}, 493-505$

Frerichs I, Schiffmann H, Hahn G, Hellige G (2001):

Non-invasive radiation-free monitoring of regional lung ventilation in critically ill infants. Intensive Care Med $\underline{27}, 1385-1394$

Frerichs I, Hinz J, Herrmann P, Weisser G, Hahn G, Dudykevych T, Quintel M, Hellige G (2002):

Detection of local lung air content by electrical impedance tomography compared with electron beam CT. J Appl Physiol 93, 660-666

Frerichs I, Schiffmann H, Oehler R, Dudykevych T, Hahn G, Hinz J, Hellige G (2003a):

Distribution of lung ventilation in spontaneously breathing neonates lying in different body positions. Intensive Care Med 29 $\underline{9}$, 787-794

Frerichs I, Dargaville P, Dudykevych T, Rimensberger P (2003b):

Electrical impedance tomography: a method for monitoring regional lung aeration and tidal volume distribution. Intensive Care Med 29, 2312-2316

Frerichs I, Braun P, Dudykevych T, Hahn G, Genée D, Hellige G (2004):

Distribution of ventilation in young and elderly adults determined by electrical impedance tomography. Respir Physiol Neurobiol 143, 63-75

Frerichs I, Schiffmann H, Hahn G, Dudykevych T, Just A, Hellige G (2005):

Funktionelle elektrische Impedanztomographie - eine Methode zur bettseitigen Überwachung der regionalen Lungenfunktion. Intensivmedizin $\underline{42}, 66-73$

Frerichs I, Schmitz G, Pulletz S, Schädler D, Zick G, Scholz J, Weiler N (2007):

Reproducibility of regional lung ventilation distribution determined by electrical impedance tomography during mechanical ventilation. Physiol Meas $\underline{28}, 261-$ 267 
Froese AB, Bryan AC (1974):

Effects of anesthesia and paralysis on diaphragmatic mechanics in man. Anesthesiology $\underline{41}$, 242-255

Gaultier C (1995):

Respiratory muscle function in infants. Eur Respir J $\underline{8}, 150-153$

Geddes LA, Baker LE (1967):

The specific resistance of biological material-a compendium of data for the biomedical engineer and physiologist. Med Biol Eng $\underline{5}, 271-293$

Griffiths H, Leung HTL, Williams RJ (1992):

Imaging the complex impedance of the thorax. Clin Phys Physiol Meas $\underline{13}$, Suppl A, 77-81

Gunnarsson L, Lindberg P, Tokics L, Thorstensson Ö, Thörne A (1995):

Lung function after open versus laparoscopic cholecystectomy. Acta Anaesthesiol Scand 39, 302-306

Hahn G, Šipinková I, Baisch F, Hellige G (1995):

Changes in the thoracic impedance distribution under different ventilatory conditions. Physiol Meas $\underline{16}, 161-173$

Hahn G, Frerichs I, Kleyer M, Hellige G (1996):

Local mechanics of the lung tissue determined by functional EIT. Physiol Meas $\underline{17}, 159-166$

Hahn G, Hartung C, Hellige G:

Elektrische Impedanztomographie (EIT) als Methode zur regionalen Beurteilung der Lungenventilation. Gustav Fischer, Mainz 1998 
Hahn G, Thiel F, Dudykevych T, Frerichs I, Gersing E, Schröder T, Hartung C, Hellige G (2001):

Quantitative evaluation of the performance of different electrical tomography devices. Biomed Tech $\underline{46}$, 91-95

Hahn G, Just A, Dudykevych T, Frerichs I, Hinz J, Quintel M, Hellige G (2006):

Imaging pathologic pulmonary air and fluid accumulation by functional and absolute EIT. Physiol Meas 27, 187-198

Hampshire AR, Smallwood RH, Brown BH, Primhak RA (1995):

Multifrequency and parametric EIT images of neonatal lungs. Physiol Meas $\underline{16}$, 175-189

Harris ND, Sugget AJ, Barber DC, Brown BH (1987):

Application of applied potential tomography (APT) in respiratory medicine. Clin Phys Physiol Meas $\underline{8}$, Suppl A, 155-165

Harris ND, Suggett AJ, Barber DC, Brown BH (1988):

Applied potential tomography: a new technique for monitoring pulmonary function. Clin Physiol Meas $\underline{9}$, Suppl A, 79-85

Heaf DP, Helms P, Gordon I, Turner HM (1983):

Postural effects on gas exchange in infants. N Engl J Med 308, 1505-1508

Heinrich S, Schiffmann H, Frerichs A, Klockgether-Radke A, Frerichs I (2006):

Body and head position effects on regional ventilation in infants: An electrical impedance tomography study. Intensive Care Med 32, 1392-1398

Hendersson RP, Webster JG (1978):

An impedance camera for spatially specific measurements of the thorax. IEEE Trans Biomed Eng 25, 250-254 
Hinz J, Hahn G, Neumann P, Sydow M, Mohrenweiser P, Hellige G, Burchardi H (2003a):

End-expiratory lung impedance change enables bedside monitoring of endexpiratory lung volume change. Intensive Care Med 29, 37-43

Hinz J, Neumann P, Dudykevych T, Andersson LG, Wrigge H, Burchardi $H$, Hedenstierna G (2003b):

Regional ventilation by electrical impedance tomography: a comparison with ventilation scintigraphy in pigs. Chest $\underline{124}, 314-322$

Hinz J, Moerer O, Neumann P, Dudykevych T, Hellige G, Quintel M (2005):

Effect of positive end-expiratory-pressure on regional ventilation in patients with acute lung injury evaluated by electrical impedance tomography. Eur $\mathrm{J}$ Anaesthesiol $\underline{22}, 817-825$

Hislop A, Reid L:

Growth and development of the respiratory system: Anatomical development. In: Scientific Foundation of Pediatrics, $2^{\text {nd }}$ edition, hrsg. v. J.A. Davis, J. Dobbing. Heinemann Medical Publications, London 1981, 390-431

Holder DS (1989):

Impedance changes during evoked nervous activity in human subjects: implications for the application of applied potential tomography (APT) to imaging neuronal discharge. Clin Phys Physiol Res $\underline{10}$, 267-274

Holder DS, Rao A, Hanquan Y (1996):

Imaging of physiologically evoked responses by electrical impadance tomography with cortical electrodes in anaesthetized rabbit. Physiol Meas $\underline{17}$, 179-186 
Holder DS, González-Correa CA, Tidswell T, Gibson A, Cusick G, Bayford RH (1999):

Assessment and calibration of a low-frequency system for electrical impedance tomography (EIT), optimized for use in imaging brain function in ambulant human subjects. Ann N Y Acad Sci $\underline{873}, 512-519$

Howatt WF, Talner NS, Sloan H (1962):

Pulmonary function changes following repair of heart lesions with the aid of extracorporeal circulation. J Thorac Cardiovasc Surg $\underline{43}, 649-657$

Humphreys S, Pham T, Stocker C, Schibler A (2011):

The effect of induction of anesthesia and intubation on end-expiratory lung level and regional ventilation distribution in cardiac children. Pediatr Anaesth $\underline{21}, 887-893$

Johnson D, Hurst T, Thomson D, Mycyk T, Burbridge T, To T, Mayers I (1996):

Respiratory function after cardiac surgery. J Cardiothorac Vasc Anesth 10, 571-577

Kaneko K., Milic-Emili J., Dolovich MB., Dawson A., Bates DV. (1966):

Regional distribution of ventilation and perfusion as a function of body position. J Appl Physiol 21, 767-777

Karsten J, Luepschen H, Grossherr M, Bruch HP, Leonhardt S, Gehring H, Meier T (2011):

Effect of PEEP on regional ventilation during laparoscopic surgery monitored by electrical impedance tomography. Acta Anaesthesiol Scand $\underline{55}, 878-886$

Kristjánsdóttir A, Ragnarsdóttir M, Hannesson P, Beck HJ, Torfason B, (2004):

Respiratory movements are altered three months and one year following cardiac surgery. Scand Cardiovasc J $\underline{38}, 98-103$ 
Kunst PW, Noordegraf AV, Hoekstra OS, Postmus PE, de Vries PMJM (1998a):

Ventilation and perfusion imaging by electrical impedance tomography: a comparison with radionuclide scanning. Physiol Meas $\underline{19}$, 481-490

Kunst PW, Vonk Noordegraf A, Straver B, Aarts RAHM, Tesselaar CD, Postmus PE, de Vries PMJM (1998b):

Influences of lung parenchyma density and thoracic fluid on ventilatory EIT measurements. Physiol Meas $\underline{19}$, 27-34

Kunst PW, de Vries PM, Postmus PE, Bakker J (1999a):

Evaluation of electrical impedance tomography in the measurement of PEEPinduced changes in lung volume. Chest $\underline{115}, 1102-1106$

Kunst PW, Noordegraaf AV, Raaijmakers E, Bakker J, Groeneveld AB, Postmus PE, de Vries PM (1999b):

Electrical impedance tomography in the assessment of extravascular lung water in noncardiogenic acute respiratory failure. Chest $\underline{116}, 1695-1702$

Kunst PW, Böhm SH, Vazquez de Anda G, Amato MB, Lachmann B, Postmus PE, de Vries PM (2000a):

Regional pressure volume curves by electrical impedance tomography in a model of acute lung injury. Crit Care Med $\underline{28}, 178-183$

Kunst PW, Vazquez de Anda G, Böhm SH, Faes TJ, Lachmann B, Postmus PE, de Vries PM (2000b):

Monitoring of recruitment and derecruitment by electrical impedance tomography in a model of acute lung injury. Crit Care Med $\underline{28}, 3891-3895$

Lindgren S, Odenstedt H, Olegard C, Söndergaard S, Lundin S, Stenqvist O (2007):

Regional lung derecruitment after endotracheal suction during volume- or pressure-controlled ventilation: a study using electric impedance tomography. Intensive Care Med $\underline{33}, 172-180$ 
Locke TJ, Griffiths TL, Mould H, Gibson GJ (1990):

Rib cage mechanics after median sternotomy. Thorax $\underline{45}, 465-468$

Lowhagen K, Lindgren S, Odenstedt H, Stenquist O, Lundin S (2011):

A nex nonradiological method to assess potential lung recruitability: a pilot study in ALI patients. Acta Anaesthesiol Scand $\underline{55}$, 165-174

Marquis F, Coulombe N, Costa R, Gagnon H, Guardo R, Skrobik Y (2006):

Electrical impedance tomography's correlation to lung volume is not influenced by anthropometric parameters. J Clin Monit Comput 20, 201-207

Marven SS, Hampshire AR, Smallwood RH, Brown BH, Prim hak RA (1996):

Reproducibility of electrical impedance tomographic spectroscopy (EITS) parametric images of neonatal lungs. Physiol Meas $\underline{17}$, Suppl 4A, 205-212

Mc Ardle FJ, Sugget AJ, Brown BH, Barber DC (1988):

An assessment of dynamic images by applied potential tomography for monitoring pulmonary perfusion. Clin Phys Physiol Meas $\underline{9}$, Suppl A, 87-91

Meier T, Leibecke T, Eckmann C, Gosch UW, Grossherr M, Bruch HP, Gehriing H, Leonhardt S (2006):

Electrical impedance tomography: changes in distribution of pulmonary ventilation during laparoscopic surgery in a porcine model. Langenbecks Arch Surg $\underline{391}, 383-389$

Meier T, Luepschen H, Karsten J, Leibecke T, Grossherr M, Gehring H, Leonhardt S (2008):

Assessment of regional lung recruitment and derecruitment during a PEEP trial based on electrical impedance tomography. Intensive Care Med 34, 543550 
Morice AH, Harris N, Campbell J, Zhang F, Brown BH:

EIT in the investigation of chest disease. In: Clinical and Physiological Applications of Electrical Impedance Tomography; hrsg. v. Holder D; UCL Press, London 1993, 236-241

Muller NL, Bryan AC (1979):

Chest wall mechanics and respiratory muscles in infants. Pediatr Clin North Am $\underline{26}, 503-516$

Murphy D, Burton P, Coombs R, Tarassenko L, Rolfe P (1987):

Impedance imaging in the newborn. Clin Phys Physiol Meas $\underline{8}$, Suppl A, 131140

Neumann P, Berglund JE, Mondejar EF, Magnusson A, Hedenstierna G (1998):

Dynamics of lung collapse and recruitment during prolonged breathing in porcine lung injury. J Appl Physiol $\underline{85}$, 1533-1543

Oczenski W:

Atmen - Atemhilfen, Atemphysiologie und Beatmungstechnik, 8., überarbeitete

Auflage; hrsg. v. Oczenski W, Andel H, Werba A; Georg Thieme Verlag,

Stuttgart, Wien 2008, 10-128

Openshaw P, Edwards S, Helms P (1984):

Changes in rib cage geometry during childhood. Thorax $\underline{39}, 624-627$

Pham TM, Yuill M, Dakin C, Schibler A (2011):

Regional ventilation distribution in the first six months of live. Eur Respir J. $\underline{37}$, 919-924

Polese G, Lubli P, Mazzucco A, Luzzani L, Rossi A (1999):

Effects of open heart surgery on respiratory mechanics. Int Care Med $\underline{25}$, 1092-1099 
Putensen-Himmer G, Putensen C, Lammer H, Lingau W, Aigner F, Benzer H (1992): Comparison of postoperative respiratory function after laparoscopy or open laparotomie for cholecystectomie. Anaesthesiology $\underline{77}, 675-680$

Reid L (1984):

Lung growth in health and disease. Br J Dis Chest $\underline{78}, 113-134$

Reifferscheid F, Elke G, Pulletz S, Gawelczyk B, Lautenschläger I, Steinfath M, Weiler N, Frerichs I (2011):

Regional ventilation distribution determined by electrical impedance tomography: Reproducibility and effects of posture and chest plane. Respirology 16, 523-531

Richard JC, Pouzot C, Gros A, Tourevieille C, Lebars D, Lavenne F, Frerichs I, Guérin C (2009):

Electrical impedance tomography compared to positron emission tomography for the measurement of regional lung ventilation: an experimental study. Crit Care $\underline{13}, \mathrm{R} 82$

Richards CC, Bachmann L (1961):

Lung and chest wall compliance of apneic paralyzed infants. J Clin Invest $\underline{40}$, 273-278

Riedel T, Kyburz M, Latzin P, Thamrin C, Frey U (2009):

Regional and overall ventilation inhomogeneities in preterm and term-born infants. Intensive Care Med $\underline{35}, 144-151$

Rooney D, Friese M, Fraser JF, R Dunster K, Schibler A (2009):

Gravity-dependent ventilation distribution in rats measured with electrical impedance tomography. Physiol Meas. $\underline{30}$, 1075-85

Schibler A, Yuill M, Parsley C, Pham T, Gilshenan K, Dakin C (2009):

Regional ventilation distribution in non-sedated spontaneously breathing newborns and adults is not different. Pediatr Pulmonol $\underline{44}$, 851-858 
Serrano RE, de LB, Casas O, Feixas T, Calaf N, Camacho V, Carrió I, Casan P, Sanchis J, Riu PJ (2002):

Use of electrical impedance tomography for the assessment of unilateral pulmonary function. Physiol Meas $\underline{23}, 211-20$

Smallwood RH, Nour S, Mangnall Y, Smythe A:

Impedance imaging and gastric motility. In: Clinical and Physiological Applications of Electrical Impedance Tomography; hrsg. v. Holder D; UCL Press, London 1993, 145-153

Smallwood RH, Hampshire AR, Brown BH, Primhak RA, Marven S, Nopp P (1999):

A comparison of neonatal and adult lung impedances derived from EIT images. Physiol Meas $\underline{20}, 401-413$

Smith I, Fleming S, Cernaianu A (1990):

Mishaps during transport from the intensive care unit. Crit Care Med $\underline{18}$, 278281

Stock MC, Downs JB, Weaver D (1986):

Effect of pleurotomy on pulmonary function after median sternotomy. Ann Thorac Surg $\underline{42}, 441-444$

Taktak A, Spencer A, Record P, Gadd R, Rolfe R (1996):

Feasibility of neonatal lung imaging using electrical impedance tomography. Early Human Dev $\underline{44}, 131-138$

Thurlbeck WM (1975):

Postnatal growth and development of the lung. Am Rev Resp Dis 111, 803944

Vaisman N, Weintrob N, Blumental A, Yosefsberg Z, Vardi P (1999):

Gastric emptying patients with type I diabetes mellitus. Ann N Y Acad Sci $\underline{873}$, 506-511 
Van Genderingen HR, Vught AJ, Jansen JR (2003):

Estimation of regional lung volume changes by electrical impedance tomography during a pressure-volume manoever. Intensive Care Med $\underline{29}$, 233240

Van Genderingen HR, van Vught AJ, Jansen JR (2004):

Regional lung volume during high-frequency oscillatory ventilation by electrical impedance tomography. Crit Care Med $\underline{32}, 787-794$

Victorino JA, Borges JB, Okamoto VN, Matos GF, Tucci MR, Caramez MP, Tanaka H, Suarez-Sipmann F, Santos DC, Barbas CS, Carvalho CR, Amato MB (2004): Imbalances in regional lung ventilation: a validation study on electrical impedance tomography. Am J Respir Crit Care Med 169, 791-800

Vonk Noordegraaf A, Faes TJC, Janse A, Marcus JT, Heethar RM, Postmus PE, de Vries PMJM (1996):

Improvement of cardiac imaging in electrical impedance tomography by means of a new electrode configuration. Physiol Meas $\underline{17}, 179-188$

Warren J, Fromm RE Jr, Orr RA, Rotello RC, Horst HM (2004):

Guidelines for the inter- and intrahospital transport of critically ill patients. Crit Care Med 32, 256-262

Wrigge H, Zinserling J, Muders T, Varelmann D, Günther U, von der Groeben C, Magnusson A, Hedenstierna G, Putensen C (2008):

Electrical impedance tomography compared with thoracic computed tomography during a slow inflation manoeuver in experimental models of lung injury. Crit Care Med 36, 903-909 


\section{Liste der Abkürzungen}

Abb.

AF

Al

AMV

ANOVA

APT

art.

ASD

atyp.

AVSD

BIPAP

BTA

bzw.

$\mathrm{Cl}$

$\mathrm{cm}$

$\mathrm{CO}_{2}$

CPAP

CT

d

d.h.

$\Delta \mathrm{Z}$

d-MGA

DORV

d-TGA

EIT

EKG

et al.

$\mathrm{FiO}_{2}$

FRC

Geschl.
Abbildung

Atemfrequenz

Aorteninsuffizienz

Atemminutenvolumen

Analysis of Variance

Applied Potential Tomography

arteriell

Vorhofseptumdefekt

atypisch

atrioventrikulärer Septumdefekt

Biphasic Positive Airway Pressure (biphasischer positiver Atemwegsdruck)

Blalock-Taussig-Anastomose

beziehungsweise

Konfidenzintervall

Zentimeter

Kohlendioxid

Continuous Positive Airway Pressure (kontinuierlicher positiver Atemwegsdruck)

Computertomographie

Tag

das heißt

Impedanzänderung

d-Malposition der großen Arterien

Double Outlet Right Ventricle

d-Transposition der großen Arterien

elektrische Impedanztomographie

Elektrokardiogramm

und andere

Sauerstoffanteil

funktionelle Residualkapazität

Geschlecht 


\begin{tabular}{|c|c|}
\hline GLM & Generalized Linear Model \\
\hline GLMRM & Generalized Linear Model for Repeated Measurements \\
\hline $\mathrm{h}, \mathrm{hh}$ & Stunde \\
\hline $\mathrm{Hz}$ & Hertz \\
\hline 1 & elektrischer Strom \\
\hline ISTA & Aortenisthmusstenose \\
\hline $\mathrm{kg}$ & Kilogramm \\
\hline $\mathrm{kHz}$ & Kilohertz \\
\hline LPA & linke Pulmonalarterie \\
\hline $\mathrm{m}$ & männlich \\
\hline $\mathrm{mA}$ & Milliampère \\
\hline mbar & Millibar \\
\hline MI & Mitralinsuffizienz \\
\hline Min., mm & Minute \\
\hline $\mathrm{mmHg}$ & Millimeter-Quecksilbersäule \\
\hline MRT & Magnet-Resonanz-Tomographie \\
\hline $\mathrm{O}_{2}$ & Sauerstoff \\
\hline $\mathrm{OP}$ & Operation \\
\hline PA & Pulmonalarterie \\
\hline $\mathrm{P}_{\mathrm{a}} \mathrm{CO}_{2}$ & Kohlendoixid-Partialdruck im arteriellen Blut \\
\hline $\mathrm{P}_{\mathrm{a}} \mathrm{O}_{2}$ & Sauerstoff-Partialdruck im arteriellen Blut \\
\hline PEEP & $\begin{array}{l}\text { Positive End-Expiratory Pressure (positiver endexspiratorischer } \\
\text { Druck) }\end{array}$ \\
\hline PET & Positronen-Emissions-Tomographie \\
\hline PFO & persistierendes Foramen ovale \\
\hline$P_{\text {insp }}$ & Inspirationsdruck \\
\hline PS & Pulmonalstenose \\
\hline rel. & relativ \\
\hline rel. $\Delta Z$ & relative Impedanzänderung \\
\hline RV-PA & rechter Ventrikel zur Pulmonalarterie \\
\hline $\mathrm{S}_{\mathrm{a}} \mathrm{O}_{2}$ & Sauerstoffsättigung \\
\hline SD & Standardabweichung \\
\hline Sec, ss & Sekunde \\
\hline
\end{tabular}


SIMV

Synchronized Intermittent Mandatory Ventilation (synchronisierte intermittierend mandatorische Ventilation)

S.o. siehe oben

SPECT

Single-Photon-Emissions-Computertomographie

Tab.

Tabelle

$\mathrm{T}_{\text {insp }}$

Inspirationszeit

U

Spannung

V.a.

Verdacht auf

vgl.

vergleiche

VSD

Ventrikelseptumdefekt

w

weiblich

z.B.

zum Beispiel

Z.n.

Zustand nach 


\section{Danksagung}

Meinem Doktorvater Herrn Prof. Dr. med. T. Paul, Direktor der Abteilung Pädiatrie III (Schwerpunkt pädiatrische Kardiologie und Intensivmedizin) im Zentrum Kinderheilkunde und Jugendmedizin der Universitätsmedizin Göttingen, danke ich ganz herzlich für die Ermöglichung der Promotion und die Überlassung des Themas.

Zudem möchte ich Herrn Dr. med. U. Krause für die gute Betreuung danken, dabei vor allem für die praktische Anleitung der Durchführung der Messungen auf der Intensivstation, für die Unterstützung bei der Rekrutierung der Patienten, für entscheidende Hilfestellungen bei der Planung der Studie, für die wertvollen Diskussionen und Ratschläge bei der Fertigstellung der Arbeit sowie die stets unkomplizierte Zusammenarbeit.

Ein ganz besonderes Dankeswort gilt auch Herrn Dr. rer. biol. hum. G. Hahn und Herrn Dr. rer. nat. J. Dittmar aus der Abteilung Anästhesiologie der Universitätsmedizin Göttingen. Sie haben mir die Messgeräte zur Verfügung gestellt und hatten immer ein offenes Ohr bei technischen Fragen und Problemen. Außerdem haben sie mich bei der Aufbereitung und Auswertung der Daten unterstützt. Ohne ihre Hilfe wäre es mir nicht möglich gewesen, die Arbeit in der vorliegenden Form fertig zu stellen.

Meinen Dank möchte ich auch an das gesamte Pflegepersonal der Station 0133 (pädiatrische Intensivstation) der Universitätsmedizin Göttingen richten. Sie haben mich immer sehr freundlich und geduldig empfangen und mich trotz des belastenden Arbeitsalltags auf einer pädiatrischen Intensivstation bei allen Messungen in jeder Hinsicht unterstützt.

Meinem Vater Prof. Dr. rer. nat. P.H. Becker danke ich für die statistische Beratung, meiner Mutter Gabriele Becker für das Korrekturlesen der Arbeit und meinem Freund Gerd-Marten Kuscher sowie meinem Bruder Andreas Becker für die Unterstützung bei Fragen zum Layout. 


\section{Lebenslauf}

Ich wurde am 09. Juni 1980 als erstes Kind meiner Eltern Gabriele Becker, Gymnasiallehrerin, und Prof. Dr. Peter Hermann Becker, Diplombiologe, in Wilhelmshaven geboren. Meine beiden Geschwister Andreas und Birgit Becker kamen 1982 und 1984 zur Welt.

Nach dem Besuch der Grundschule (1986-1990) und der Orientierungsstufe in Jade (1990-1992) besuchte ich das Lothar-Meyer-Gymnasium in Varel. Dort machte ich 1999 mein Abitur.

Im Anschluss daran verbrachte ich 6 Monate als Au pair in Chile (Valdivia), bevor ich von März 2000 bis März 2001 Lehramt für Grund- und Hauptschulen an der Pädagogischen Hochschule Heidelberg studierte.

Im April 2001 begann ich das Medizinstudium an der Georg-August-Universität Göttingen. Das Physikum bestand ich im April 2003, das erste Staatexamen im April 2004. Die Zweite Ärztliche Prüfung schloss ich im Juni 2008 ab.

Ich famulierte in einer allgemeinmedizinischen Praxis in Jade, in der pädiatrischen Intensivmedizin des Universitätsklinikums Göttingen, in der Pädiatrie in Chile (Valdivia) und der Anästhesie in Argentinien (Buenos Aires). Die ersten zwei Tertiale des Praktischen Jahres (Juni 2006 - April 2007) absolvierte ich in Südamerika: Innere Medizin in Argentinien (Buenos Aires) und Chirurgie in Chile (Valdivia), hierfür erhielt ich ein Stipendium des DAAD. Das dritte Tertial (April 2007 - Juli 2007) verbrachte ich in der Pädiatrie der Universitätsmedizin Göttingen.

Ende 2004 begann ich mit der Dissertationsarbeit mit dem Titel „Postoperatives Monitoring der regionalen Lungenventilation durch die Elektrische Impedanztomographie bei Kindern und Jugendlichen mit einem angeborenen Herzfehler" in der Abteilung Pädiatrie III mit Schwerpunkt Pädiatrische Kardiologie und Intensivmedizin des Zentrums Kinderheilkunde und Jugendmedizin der Universitätsmedizin Göttingen.

Seit Oktober 2008 arbeite ich als Assistenzärztin in der Pädiatrie des Kinderkrankenhauses Park Schönfeld in Kassel (Gesundheit Nordhessen).

Kassel, Januar 2012

Kristin Becker 